\title{
Abstracts of the World Congress of Enhanced Recovery After Surgery and Perioperative Medicine, Washington, DC, USA, May 9-12, 2015
}

(C) Canadian Anesthesiologists' Society 2015

\section{OR001}

\section{ADHERENCE TO THE ERAS-PROTOCOL AND 5-YEAR SURVIVAL AFTER COLORECTAL CANCER SURGERY: A RETROSPECTIVE COHORT STUDY

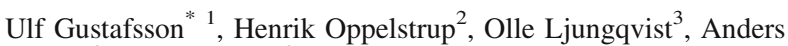 Thorell $^{2}$, Jonas Nygren ${ }^{2}$}

${ }^{1}$ Dep of Surgery, Danderyd University Hospital Department, ${ }^{2}$ Dep of Surgery, Ersta Hospital, Department of Clinical Sciences, Danderyd Hospital, Karolinska Institutet, Stockholm, Sweden, ${ }^{3}$ Dep of Surgery, Örebro University Hospital, Inst. of Molecular Medicine and Surgery, Karolinska Institutet, Stockholm, Sweden, Stockholm, Sweden

Objectives: There is evidence that surgical stress can influence oncological outcome and survival (1). This study investigated if increased adherence to the ERAS (Enhanced Recovery After Surgery) protocol, designed to reduce perioperative stress (2), is associated with increased long-term survival after surgery for colorectal cancer.

Methods: Between the years 2002-2007, 911 consecutive patients, operated with major colorectal cancer surgery at Ersta Hospital, Stockholm, Sweden were analyzed according to adherence to twelve pre- and perioperative ERAS protocol interventions. The histopathological report of the resected specimen, date and cause of death of the patients as well as post-operative CRP levels were obtained. The relation between the rate of adherence to the ERAS-protocol and five-year overall and colorectal cancer specific survival was determined.

Results: Increased adherence to ERAS-interventions was significantly associated with improved 5-year cancer specific survival in the group with adherence $>70 \%$ $(\mathrm{N}=273, \mathrm{p}<0.001)$. In Cox regression, correcting for confounding, the risk of cancer specific death was improved $42 \%$, HR $0.58(0.39-0.88)$ in this group compared to all other patients. With low adherence, $<50 \%(\mathrm{n}=317)$, the risk of cancer death increased by $83 \%$, HR $1.83(1.16-2.88)$ compared to the group with $\geq 70$ adherence. Significant independent predictors of increased 5-year survival were: avoiding overload of intravenous fluids, HR $0.528(0.32-0.86)$ and oral intake, HR $0.55(0.34-0.78)$ on the day of operation. Also, increased CRP post-operative day 1 (POD1) was found as an independent predictor for decreased 5-year survival. Patients with $\geq 70 \%$ adherence had a lower CRP, POD1 compared to all other patients, mean SD $72 \pm 36$ vs $85 \pm 41(\mathrm{p}<0.001)$

Conclusion: Increased adherence to the ERAS-protocol is associated with increased 5year cancer specific survival after colorectal cancer surgery.

Disclosure of Interest: None Declared.

\section{OR002}

\section{COMPARISON OF PATIENT REPORTED SYMPTOM BURDEN PRE- AND POST-IMPLEMENTATION OF AN ENHANCED RECOVERY PATHWAY FOR GYNECOLOGIC SURGERY}

Larissa A. Meyer ${ }^{*}{ }^{1}$, Alpa M. Nick ${ }^{1}$, Maria D. Iniesta ${ }^{1}$, Qiuling Shi ${ }^{2}$, LaKisha Washington $^{3}$, Melinda Harris ${ }^{1}$, Lindsey D. Palmer ${ }^{1}$,

Katherine E. Cain ${ }^{4}$, Terri W. Earles ${ }^{1}$, Javier Lasala ${ }^{5}$, Xin S. Wang ${ }^{2}$, Pedro T. Ramirez ${ }^{1}$

${ }^{1}$ Department of Gynecologic Oncology, ${ }^{2}$ Department of Symptoms Research, ${ }^{3}$ Department of Nursing, ${ }^{4}$ Division of Pharmacy, ${ }^{5}$ Department of Anesthesia, The University of Texas MD Anderson Cancer Center, Houston, United States

Objectives: With the growing focus on patient-centered care, patient-reported outcomes (PROs) have become an important component of comparative effectiveness research. Our objective was to compare the patient-reported symptom burden in women undergoing surgery pre- and post-implementation of an enhanced recovery pathway (ERP). Methods: Perioperative patient reported symptom burden was measured for women undergoing laparotomy on the gynecologic oncology service at the University of Texas MD An MD Anderson Cancer Center pre- and post-implementation of our ERP. Symptom were assessed using the M.D. Anderson Symptom Inventory (MDASI-OC), a 27-item
tool validated for use in patients with ovarian cancer. The MDASI-OC was administered preoperatively and daily while admitted to the hospital after surgery. Clinical data were abstracted. Fishers' exact and mixed-effect modeling were performed.

Results: Results: 51 patients ( 29 pre-ERP, 22 post-ERP) were evaluated. There was no significant difference in the top 5-reported symptoms (fatigue, abdominal pain, pain, dry mouth and drowsiness) at the preoperative baseline assessment. There was no significant difference in mean pain scores between the pre- and post enhanced recovery cohorts within 3 days after surgery despite a $81 \%$ reduction in intake of opioids (median morphine equivalents), or in the other most highly rated symptoms of fatigue, dry mouth and drowsiness. However, compared to traditional care, patients in the ERP reported significantly lower severity of nausea, sleep disturbance, constipation, urinary urgency and difficulty with memory during the hospitalization period.

Mean Pain Scores for hospitalized patients

\begin{tabular}{llllll}
\hline Symptom & Group & Pre-op & POD1 & POD2 & POD3 \\
\hline Pain & Control & 2.31 & 6.11 & 4.75 & 5.17 \\
& ERP & 3.23 & 5.05 & 4.80 & 4.73 \\
& & $\mathrm{P}=0.28$ & $\mathrm{P}=0.35$ & $\mathrm{P}=0.97$ & $\mathrm{P}=0.74$ \\
\multirow{4}{*}{ Abdominal Pain } & Control & 2.38 & 6.33 & 4.83 & 4.72 \\
& ERP & 2.86 & 5.53 & 4.73 & 4.73 \\
& & $\mathrm{P}=0.55$ & $\mathrm{P}=0.48$ & $\mathrm{P}=0.93$ & $\mathrm{P}=0.99$
\end{tabular}

Conclusion: Adoption of an enhanced recovery pathway can significantly reduce opioid consumption without adversely worsening patient subjective feeling of pain and other perioperative symptoms. The decrease in severity of nausea, sleep disturbance, constipation and memory are likely related to the decrease in opioid consumption in the immediate postoperative setting.

Disclosure of Interest: None Declared. 


\section{OR003}

\section{ADHERENCE TO THE ENHANCED RECOVERY AFTER SURGERY AND ITS ECONOMIC IMPACT}

\author{
Jakob Stenseth $^{*}{ }^{1}$, Ulf Gustafsson ${ }^{2}$, Olle Ljungqvist ${ }^{1}$ \\ ${ }^{1}$ Department of Surgery, Örebro University, Örebro, ${ }^{2}$ Karolinska \\ Institutet, Stockholm, Sweden
}

Objectives: In the ERAS protocol, a multimodal approach is taken to reduce complications and length of stay in patients undergoing elective colorectal surgery. Previous studies have shown a reduction of both aspects, more so with higher adherence to the ERAS protocol. In this study, we analyzed the economic impact of compliance to the ERAS protocol.

Methods: A cohort of 953 patients undergoing elective colorectal surgery was registered consecutively into a database. According to the severity of their complications, patients were classified using the Clavien-Dindo system. This classification has recently been used to calculate costs (1). Using a template verified in a previous study, a cost was calculated for each patient.

Results:

\begin{tabular}{llll}
\hline Adherence/Complication & $\begin{array}{l}<50 \% \\
\mathrm{n}=333\end{array}$ & $\begin{array}{l}>70 \% \\
\mathrm{n}=102\end{array}$ & $\begin{array}{l}>90 \% \\
\mathrm{n}=76\end{array}$ \\
\hline None $(\$ 26,420)$ & $29 \%$ & $49 \%$ & $66 \%$ \\
Minor $(\$ 36,268)$ & $44 \%$ & $40 \%$ & $28 \%$ \\
Major $(\$ 95,550)$ & $27 \%$ & $11 \%$ & $6 \%$ \\
Cost/patient & $\$ 48353$ & $\$ 37834$ & $\$ 33689$ \\
\hline
\end{tabular}

The table shows the frequency of complications is shown alongside the calculated average cost per patient grouped according to the different levels of adherence to the ERAS protocol. The differences between all levels of adherence $>70 \%$ compared to $<50 \%$ was shown to be significant using chi-square test for trend.

Conclusion: With higher adherence to the ERAS protocol, fewer and less severe complications were seen. This was also associated with lower cost.

Disclosure of Interest: None Declared.

\section{OR004}

\section{FURTHER VALIDATION OF THE SIX-MINUTE WALK TEST AS A MEASURE OF POSTOPERATIVE RECOVERY AFTER COLORECTAL SURGERY}

Nicolò Pecorelli ${ }^{*}{ }^{1}$, Julio F. Fiore Jr. ${ }^{1}$, Benjamin Mappin-Kasirer ${ }^{1}$, Petru Niculiseanu ${ }^{1}$, Chelsia Gillis ${ }^{2}$, Rashami Awasthi ${ }^{2}$, Francesco Carli $^{2}$, Liane S. Feldman ${ }^{1}$

${ }^{1}$ Steinberg Bernstein Centre for Minimally Invasive Surgery at McGill University, ${ }^{2}$ Anesthesia, McGill University Health Centre, Montreal, Canada

Objectives: To contribute further evidence for the construct validity of the 6-minute walk test (6MWT) as a measure of postoperative recovery after colorectal surgery.

Methods: Patients from three recently completed randomized controlled trials investigating interventions to improve recovery after colorectal resection were considered for the study. Patients who did not perform the 6MWT within 4 weeks before surgery, and at 4 weeks postoperatively were excluded. Construct validity was assessed by testing the hypotheses that 6 minute walk distance (6MWD) at 4 weeks after surgery: (1) is greater in younger vs. older patients ( $\geq 75$ years old); (2) is greater in patients with higher preoperative physical status (ASA $<2$ ) vs. lower (ASA $\geq 3$ ); (3) is greater after laparoscopic vs. open surgery; (4) is greater in patients who experienced Clavien grade 0-II postoperative complications vs. those who had major complications (grade III-IV); (5) correlates cross-sectionally with self-reported physical activity as measured by a physical activity questionnaire (CHAMPS, kcal/kg/wk). Statistical analyses were performed using linear regression (hypotheses 1-4), and Spearman's correlation (hypothesis 5). Results are reported according to the COSMIN checklist for evaluating the quality of studies on measurement properties.

Results: 151 of 173 patients (87\%) were included in the study $(65 \%$ male; mean age 67.3 (SD 10.6) years, 91\% laparoscopic, 93\% malignancy, $41 \%$ rectal cancer). All hypotheses tested for construct validity were supported by the data (Table). 6MWD positively correlated $(r=0.46)$ with patient reported physical activity at 4 weeks after surgery.

\begin{tabular}{lcc}
\hline Comparisons & Coefficient & 95\% C.I. \\
\hline Younger vs. older patients* & 28.3 & $-8.1-64.7$ \\
Higher vs. lower physical status* & 40.4 & $11.6-69.2$ \\
Laparoscopic vs. open surgery* & 58.8 & $11.5-106.0$ \\
$\begin{array}{l}\text { Grade 0-II vs. Grade III-IV postoperative } \\
\quad \text { complications* }\end{array}$ & 101.6 & $54.2-149.0$ \\
\hline
\end{tabular}

*should be interpreted as mean difference (meters) between groups 4 weeks after surgery; regression analysis adjusted for preoperative 6MWD

Conclusion: This study contributes evidence to the validity of the $6 \mathrm{MWT}$ as a measure of postoperative recovery after colorectal surgery, and should encourage its use as an outcome measure of postoperative physical performance.

Disclosure of Interest: None Declared. 


\section{OR005}

\section{RETURN ON INVESTMENT FOR IMPLEMENTATION OF PERIOPERATIVE GOAL DIRECTED THERAPY (PGDT) IN MAJOR SURGERY: A NATIONWIDE DATABASE STUDY}

Frederic Michard ${ }^{*}$, William K. Mountford ${ }^{2}$, Michelle R. Krukas ${ }^{2}$, Frank R. Ernst ${ }^{2}$, Sandy L. Fogel ${ }^{3}$

${ }^{1}$ Edwards Lifesciences, NYON, Switzerland, ${ }^{2}$ Premier Inc., Charlotte, ${ }^{3}$ Surgery, Carilion Clinic, Roanoke, United States

Objectives: Preventable post surgical complications are increasingly recognized as a major health care burden. A recent meta-analysis (Pearse et al. JAMA 2014) showed a 17-29\% decrease in complications after major surgery with PGDT. We assessed the financial consequences of postsurgical complications in a large population from 541 US hospitals in order to predict potential savings with PGDT.

Methods: Data from adults who had any one of 10 major non-cardiac surgical procedures between January 2011 and June 2013 were selected from the Premier research database. Twenty-six post-surgical complications were tabulated. Hospital costs, length of stay, and readmission rates were compared in patients with and without complications. Risk ratios reported by Pearse's meta-analysis were used to estimate the expected reduction in post-surgical morbidity with PGDT. Potential cost-savings were calculated from the actual and anticipated morbidity rates using the mean difference in total costs.

Results: A total of 204,680 patients met the search criteria, and 76,807 patients developed one or more post-surgical complications (morbidity rate $37.5 \%$ ). In patients with and without complications, hospital costs (including 30 days readmission costs) were $\$ 27,607 \pm 32,788$ and $\$ 15,783 \pm 12,282(\mathrm{p}<0.0001)$, median [Interquartile range] hospital lengths of stay (1st stay) were 7 [4-10] days and 4 [3-5] days $(\mathrm{p}<0.0001)$, and 30 day readmission rates were 17.2 and $11.9 \%(\mathrm{p}<0.0001)$, respectively. With PGDT, morbidity rate was anticipated to decrease to $26.6-31.1 \%$, yielding gross costs savings of $\$ 153 \mathrm{M}-\$ 263 \mathrm{M}$ for the study period, $\$ 61-\$ 105 \mathrm{M}$ per year, or $\$ 754-\$ 1,286$ per patient.

Conclusion: Postsurgical complications occurred in more than one third of our study population and had a dramatic impact on hospital costs, length of stay, and readmission rates. Potential cost savings with PGDT were \$754-\$1,286 per patient. These projections should help hospitals estimate the return on investment for implementation of PGDT

Disclosure of Interest: F. Michard Other: employee of Edwards, W. Mountford: None Declared, M. Krukas: None Declared, F. Ernst: None Declared, S. Fogel: None Declared.

\section{OR006}

\section{THE USE OF ARTIFICIAL NEURAL NETWORKS TO PREDICT ENHANCED RECOVERY OUTCOME FOLLOWING LAPAROSCOPIC COLORECTAL CANCER SURGERY}

Nader Francis ${ }^{*}{ }^{1}$, Lison Luther ${ }^{1}$, Emad Salib ${ }^{2}$, Lucy Allenby ${ }^{1}$, David Messenger $^{1}$, Andrew Allison ${ }^{1}$, Neil Smart ${ }^{3}$, Jonathan Ockrim ${ }^{1}$

${ }^{1}$ Surgery, Yeovil Hospital NHS Foundation Trust, YEOVIL,

${ }^{2}$ University of Liverpool, Liverpool, ${ }^{3}$ Surgery, Royal Devon \& Exeter NHS Foundation Trust, Exeter, United Kingdom

Objectives: Artificial neural networks (ANNs) can be used to develop predictive tools to enable the clinical decision-making process. This study aimed to investigate the use of an ANN in predicting the outcomes from enhanced recovery after colorectal cancer surgery.

Methods: Data was obtained from consecutive colorectal cancer patients undergoing laparoscopic surgery within the ERAS programme between 2002 and 2009 in a single centre. The primary outcomes assessed were delayed discharge and readmission within a 30 day period. The data was analysed using a Multi Layered Perceptron Neural Network (MLPNN), and a prediction tools were created for each outcomes. The results were compared with a conventional statistical method using Logistic regression analysis.

Results: A total of 275 cancer patients were included in the study. The median length of stay was 6 days (range: 2-49 days) with 67 patients $(24.4 \%)$ staying longer than 7 days. Thirty four patients $(12.5 \%)$ were readmitted within 30 days. Important factors predicting delayed discharge were related to failure in compliance with ERAS, particularly with the postoperative elements in the first 48 hours. The MLPNN for delayed discharge had an area under a receiver-operator characteristic curve (AUROC) of 0.817, compared with an AUROC of 0.807 for the predictive tool developed from logistic regression analysis. Factors predicting 30 day readmission included overall compliance with the ERAS pathway and receiving neoadjuvant treatment for rectal cancer. The MLPNN for readmission had an AUROC of 0.68 .

Conclusion: These results may plausibly suggest that ANN can be used to develop reliable outcome predictive tools in multi-factorial intervention such as ERAS. Compliance with ERAS can reliably predict both delayed discharge and 30 day readmission following laparoscopic colorectal cancer surgery.

Disclosure of Interest: None Declared. 


\section{PO001}

\section{ERAS PROGRAM ENHANCES FUNCTIONAL RECOVERY AFTER ROBOTIC GASTRECTOMY}

Shu Zhang ${ }^{*}{ }^{1}$, Gang Wang ${ }^{1}$, Zhi-Wei Jiang ${ }^{1}$, Kun Zhao ${ }^{1}$, Jie-shou Li $^{1}$

${ }^{1}$ Research Institute of General Surgery, Jinling Hospital, Medical School, Nanjing University, Nanjing, China

Objectives: Enhance Recovery After Surgery (ERAS) program have demonstrated advantages over traditional perioperative care after gastrectomies, and robotic surgeries also have shown minimal invasive advantages in gastric cancer patients; however, there is no investigation on Robotic Gastrectomy applied with ERAS program. This study was conducted to estimate the value of the combination of ERAS program and robotic surgery for gastric patients.

Methods: This is a randomized prospective controlled clinical trial. 177 consecutive patients underwent elective robotic gastrectomy between February 2012 and Dec 2014. 86 patients received ERAS program as ERAS group and 91 patients underwent traditional perioperative care as non-ERAS group. Postoperative hospital stay, return of gastrointestinal function, postoperative complications, 30-day readmission were recorded.

Results: Postoperative hospital stay was shorter in the robotic ERAS group, a mean duration of 4.9 days versus 6.1 days in the robotic nonERAS group $(p<0.01)$. Gastrointestinal functional recovery occurred 1 day earlier in ERAS group (passage of flatus after 2.2 days vs. 3.4 days, $\mathrm{p}<0.01$ ). There were no significant differences in complications within postoperative 30-day follow up.

\begin{tabular}{llll}
\hline & $\begin{array}{l}\text { ERAS } \\
\text { group } \\
\mathrm{n}=86\end{array}$ & $\begin{array}{l}\text { Non-ERAS } \\
\mathrm{n}=91\end{array}$ & group $P$ value \\
\hline $\begin{array}{l}\text { Walk on surgery day } \\
(\%)\end{array}$ & $10(12 \%)$ & $0(0 \%)$ & $\mathrm{P}=0.001$ \\
Walk on POD1 (\%) & $66(77 \%)$ & $21(23 \%)$ & $\mathrm{P}=0.000$ \\
Walk on POD2 (\%) & $78(91 \%)$ & $46(50 \%)$ & $\mathrm{P}=0.001$ \\
Walk on POD3 (\%) & $85(99 \%)$ & $64(71 \%)$ & $\mathrm{P}=0.001$ \\
$\begin{array}{l}\text { Days until flatus } \\
\text { Mean value, d (SD) }\end{array}$ & $2.2 \pm 2.0$ & $3.4 \pm 2.5$ & $\mathrm{P}=0.001$ \\
Median, d (range) & $2(1-6)$ & $3(1-8)$ & - \\
$\begin{array}{l}\text { Days of hospital stay } \\
\text { Mean value, d (SD) }\end{array}$ & $4.9 \pm 3.1$ & $6.1 \pm 4.8$ & - \\
Median, d (range) & $5(2-41)$ & $6(3-55)$ & $\mathrm{P}=0.001$ \\
\hline
\end{tabular}

Conclusion: When applied after robotic Gastrectomy, ERAS program is feasible, safe and may lead to accelerated functional recovery and reductions in postoperative hospital stay.

Disclosure of Interest: None Declared.

\section{PO002}

\section{ENHANCED RECOVERY IN THORACIC SURGERY: A RISK PREDICTION MODEL FOR DELAYED DISCHARGE IN PATIENTS UNDERGOING LUNG RESECTION}

Natasha A. Joshi ${ }^{* 1}$, Timothy J. P. Batchelor ${ }^{2}$, Neil J. Rasburn ${ }^{1}$, David E. Messenger ${ }^{3}$, Matthew K. Molyneux ${ }^{1}$, Kajan Kamalanathan ${ }^{1}$, Douglas G. West ${ }^{2}$, Gianluca Casali ${ }^{2}$, Rakesh Krishnadas ${ }^{2}$, Nicholas Wharton ${ }^{1}$, Rachel Craven ${ }^{1}$, Claudia Paoloni ${ }^{1}$, Lesley Wood ${ }^{1}$, Rebecca A. Leslie ${ }^{1}$, Anne Whaley ${ }^{1}$

${ }^{1}$ Department of Anaesthesia, ${ }^{2}$ Department of Thoracic Surgery,

${ }^{3}$ Department of Colorectal Surgery, University Hospitals Bristol NHS Foundation Trust, Bristol, United Kingdom

Objectives: Increased compliance with enhanced recovery after surgery (ERAS) protocols is associated with improved short term outcomes in colorectal cancer surgery. The impact of ERAS in thoracic surgery has never been formally evaluated. The objectives of this study were to determine which elements of an ERAS programme in thoracic surgery influenced length of stay (LOS) and to produce a risk prediction model for delayed discharge.

Methods: Prospective data was collected on consecutive patients undergoing lung resection from May 2012 to June 2014. All patients followed a standardized ERAS protocol incorporating 14 nationallydefined elements. Key data fields included pathway compliance, pathophysiological and operative factors. Delayed discharge was defined as any stay beyond the median LOS. Binary logistic regression was used to identify independent risk factors for delayed discharge from which a prediction model was derived.

Results: 752 patients underwent lung resection; median LOS was 4 days (range 1-94 days). There was an inverse relationship between ERAS protocol compliance and the proportion of delayed discharges ( $\mathrm{p}<0.001)$. Older age, COPD, pre-operative counselling, regional anaesthesia, an open surgical approach, targeted post-operative nausea and vomiting (PONV) control and delayed mobilization were independent predictors of delayed discharge (all $\mathrm{p}<0.05$ ). These factors were used to derive a scoring system: $(0.005 \times$ age in years $)+(0.237 \times \mathrm{COPD})+(0.120 \times$ surgical approach $)+(0.127$ $\times$ patient counselling $)+(0.117 \times$ regional anaesthesia $)+(0.165 \times$ PONV $)-(0.215 \times$ early mobilization $)-0.379$. A value $>0$ predicted delayed discharge. The area under the receiver-operator characteristic curve was 0.77 (95\% confidence interval $0.74-0.80$ ).

\begin{tabular}{lc}
\hline $\begin{array}{l}\text { Compliance with number } \\
\text { of ERAS elements }(\%)\end{array}$ & $\begin{array}{l}\text { Delayed } \\
\text { discharges }(\%)\end{array}$ \\
\hline $9(60.0)$ & $3(60.0)$ \\
$10(66.7)$ & $20(57.1)$ \\
$11(73.3)$ & $56(41.8)$ \\
$12(80.0)$ & $123(42.7)$ \\
$13(86.7)$ & $93(35.9)$ \\
$14(93.3)$ & $2(13.3)$ \\
\hline
\end{tabular}

Conclusion: Increased compliance with ERAS elements was associated with a reduction in delayed discharges for patients undergoing lung resection. COPD, open resections and delayed mobilization had the greatest impact on LOS.

Disclosure of Interest: None Declared. 


\section{PO003}

\section{THE SUCCESSFUL SPREAD AND SUSTAINABLE IMPLEMENTATION OF A SCOTTISH NATIONAL ERAS PROGRAMME IN ORTHOPAEDICS - FOUR YEARS ON, HOW ARE WE DOING?}

David A. McDonald ${ }^{*}$, 2 , Kate James ${ }^{3}$, Jane Campbell ${ }^{3}$, Jonathan Antrobus ${ }^{4}$ on behalf of Scottish National ERAS Steering Board

${ }^{1}$ Rehabilitation, Golden Jubilee National Hospital, ${ }^{2}$ Whole System Patient Flow Programme QuEST, Scottish Government, Glasgow, ${ }^{3}$ Scottish Orthopaedic Service Development Group, Scottish Government, Edinburgh, ${ }^{4}$ Anaesthetics, Borders General Hospital, Melrose, United Kingdom

Objectives: Following development of a local ERAS programmes in elective orthopaedics for total knee and hip arthroplasty surgery (McDonald et al., 2012), the Scottish Government funded a National Programme to support uptake of ERAS principles in Orthopaedics. A Multidisciplinary Steering Board was formed to support spread of the evidence base. Tailored support was offered to individual hospital teams including visits to exemplar sites, national events and online resources. The aim of this study was to investigate the impact of the National ERAS programme on uptake of ERAS programmes and clinical outcomes over a four year period across Scotland.

Methods: A National ERAS Audit was carried out each year (2010-2013) at all 22 hospital site over a twelve week period. All consecutive patients undergoing either primary THA or TKA $(n=2938)$ were included. Local Audit Co-ordinators collected data from patient case notes and patient information systems. Demographic data, whether the patient was managed as an ERAS patient, urinary catheterisation rates, blood transfusion rates, recommencement of diet and fluids, post-operative mobilisation, length of postoperative stay and readmissions were compared over the four year period.

Results: Average age/BMI, Comorbidities were similar between both groups. By 2013, a significant $(p<0.05)$ increase in patients were managed as part of an ERAS programme, resulting in a significant improvement in a number of factors known to increase morbidity and delay rehabilitation.

\begin{tabular}{lll}
\hline & $\begin{array}{l}2010 \\
(\mathrm{n}=1345)\end{array}$ & $\begin{array}{l}2013 \\
(\mathrm{n}=1593)\end{array}$ \\
\hline Patients managed as ERAS patients & $21 \%$ & $92 \%$ \\
$\begin{array}{l}\text { Blood Transfusion (Hips/Knee) } \\
\text { Urinary Catheterisation }\end{array}$ & $19 \% / 9 \%$ & $10 \% / 4 \%$ \\
$\begin{array}{l}\text { Restarting diet on day of surgery or }<12 \mathrm{hrs} \\
\quad \text { post-op }\end{array}$ & $60 \%$ & $16 \%$ \\
$\begin{array}{l}\text { Mobilisation of patients by end first day } \\
\text { post-op }\end{array}$ & $84 \%$ & $95 \%$ \\
$\begin{array}{l}\text { IV fluids stopped on day of surgery } \\
\text { or }<12 \text { hrs post-op }\end{array}$ & $22 \%$ & $70 \%$ \\
$\begin{array}{l}\text { Use of Patient Controlled Analgesia } \\
\text { Mean post-op length of stay (days) }\end{array}$ & $49 \%$ & $14 \%$ \\
\hline
\end{tabular}

Conclusion: The National ERAS Orthopaedic programme in Scotland has successful spread and shared good practise and resultantly improved known post-operative morbidly factors resulting in a shorter hospital stay with no increase in readmissions or complications.

Disclosure of Interest: None Declared.

\section{PO004}

\section{MEASURING THE IMPACT OF ENHANCED RECOVERY IN LIVER SURGERY USING PATIENT REPORTED FUNCTIONAL OUTCOMES}

Ryan W. Day ${ }^{*}{ }^{1}$, Vijaya Gottumukkala ${ }^{2}$, Thomas A. Aloia ${ }^{1}$

${ }^{1}$ Surgical Oncology, ${ }^{2}$ Anesthesiology and Critical Care, MD Anderson Cancer Center, Houston, United States

Objectives: To compare functional outcomes for liver surgery patients (pts) treated on conventional versus enhanced recovery (ERLS) pathways.

Methods: Demographic, clinicopathologic, operative and patient reported outcomes (PRO) data for 119 hepatectomy pts were recorded in realtime. PROs were collected using the validated MD Anderson Symptom Inventory (MDASI) administered once preoperatively and postoperatively at every outpatient visit until 31 days after surgery. The survey is comprised of questions scored on 0-10 Likert scales and compared in 4 domains: total, basic symptoms, GI symptoms and life interference. The ERLS protocol included patient education, narcotic sparing preoperative/intraoperative/postoperative anesthesia/analgesia, rapid diet advancement, goal-directed fluid therapy, early ambulation and avoidance of drains and tubes.

Results: The median age was 58 years-old and 55\% of pts were male. 66 ERLS pathway pts were demographically and clinically comparable to 53 pts treated simultaneously on a conventional pathway. Complication rates were similar and there were no mortalities. Compared to conventional pathway pts, ERLS pts experienced shorter hospital LOS ( 4.8 vs. 5.9 days, $\mathrm{p}=0.038)$ and were more likely to return to baseline (RTB) for total symptom score and life interference score (Table 1). After controlling for MIS approach, magnitude of hepatectomy and epidural, the only independent predictor of RTB for life interference was treatment on the ERLS pathway $(\mathrm{p}=0.001$, OR 3.73).

Table 1

\begin{tabular}{lccc}
\hline Factor & Conventional $(\mathrm{n}=53)$ & ERLS $(\mathrm{n}=66)$ & $\begin{array}{l}p- \\
\text { value }\end{array}$ \\
\hline Age (IQR) & $59(49-67)$ & $56(46-67)$ & 0.415 \\
Male (\%) & $26(49.1)$ & $39(59.1)$ & 0.275 \\
ASA 3/4 (\%) & $50(94.3)$ & $64(97.0)$ & 0.655 \\
Preop Chemotherapy (\%) & $41(77.4)$ & $50(75.8)$ & 0.838 \\
Major Complication (\%) & $7(13.2)$ & $6(9.1)$ & 0.474 \\
Epidural (\%) & $33(62.3)$ & $30(45.5)$ & 0.068 \\
Major Hepatectomy (\%) & $18(34.0)$ & $20(30.3)$ & 0.670 \\
Minimally Invasive (\%) & $7(13.2)$ & $24(36.4)$ & 0.004 \\
RTB Total (\%) & $8(15.1)$ & $27(40.9)$ & 0.002 \\
RTB Basic (\%) & $16(30.2)$ & $30(45.5)$ & 0.089 \\
RTB GI (\%) & $29(54.7)$ & $45(68.2)$ & 0.132 \\
RTB Life Interference $(\%)$ & $13(24.5)$ & $36(54.5)$ & 0.001 \\
LOS (IQR) & $4.7(3.0-6.0)$ & $5.8(4.0-7.0)$ & 0.047 \\
\hline
\end{tabular}

Conclusion: In addition to shorter LOS, ERLS facilitates more frequent and faster return to baseline performance status and functional outcome.

Disclosure of Interest: None Declared. 


\section{PO005}

\section{SYSTEMATIC REVIEW AND META-ANALYSIS OF THE USE OF POSTOPERATIVE CHEWING GUM TO PREVENT ILEUS}

Vaneesha Short ${ }^{*}$, Georgia Herbert ${ }^{1}$, Rachel Perry ${ }^{1}$, Charlotte Atkinson $^{1}$, Andy Ness ${ }^{1}$, Christopher Penfold ${ }^{1}$, Steven Thomas ${ }^{2}$, Henning K. Andersen ${ }^{3}$, Stephen Lewis ${ }^{4}$

${ }^{1}$ NIHR BRU in Nutrition, Diet and Lifestyle, University of Bristol, ${ }^{2}$ Head \& Neck Surgery, University Hospitals Bristol NHS Trust, Bristol, United Kingdom, ${ }^{3}$ Colorectal Cancer Group, Bispebjerg Hospital, Copenhagen, Denmark, ${ }^{4}$ Department of Gastroenterology, Derriford Hospital, Plymouth, United Kingdom

Objectives: Ileus commonly occurs after abdominal surgery, and is associated with increased length of hospital stay (LOHS). We explored whether postoperative chewing gum $(\mathrm{CG})$ reduces ileus by accelerating return of gastrointestinal (GI) function.

Methods: We searched six electronic databases and hand-searched for randomised-controlled trials (RCTs) on the use of postoperative CG in abdominal surgery patients. We split studies into colorectal surgery, caesarean section and other surgery subgroups. We assessed risk of bias (ROB) using an adapted Cochrane ROB tool. We assessed effects of CG on time to first flatus (TFF), time to bowel movement (TBM) and LOHS via random-effects model meta-analyses. We also considered the effect of CG on complications and conducted a sensitivity analysis in studies within an Enhanced Recovery After Surgery (ERAS) context.

Results: We found 81 eligible studies that recruited 9072 participants to include in our review. Many studies were at high or unclear ROB. There was statistical evidence that compared to controls, use of CG reduced TFF overall by 10.4 hours $(95 \%$ CI: $-11.9,-8.9)$, TBM by 12.7 hours $(95 \%$ CI: $-14.5,-10.9)$ and LOHS by 0.7 days $(95 \%$ CI: $-0.8,-0.5)$. There was statistical evidence of heterogeneity in all analyses, and visual inspection of the funnel plots indicated that reporting bias may be present for TBM and LOHS. Effect sizes were greatest in the colorectal surgery subgroup and smallest in the caesarean section subgroup. There was little difference between treatment groups in mortality, infection and readmissions. The ERAS sensitivity analysis (four studies) showed that compared to controls, use of CG reduced TFF by 6.2 hours $(95 \% \mathrm{CI}:-15.4,3.0)$ and TBM by 21.1 hours $(95 \% \mathrm{CI}:-33.1,-9.2)$, but did not affect LOHS (increase of 0.1 days, $95 \% \mathrm{CI}$ : $-0.4,0.5$ ).

Conclusion: We found some evidence for a benefit of postoperative $\mathrm{CG}$ on GI function recovery, although many trials were small and of poor quality. Many components of ERAS reduce risk of ileus, which may explain the apparent reduction in effectiveness of CG alongside ERAS programmes. Well-designed adequately-powered RCTs are needed to demonstrate the effectiveness of CG after surgery in contemporary populations in ERAS settings.

Disclosure of Interest: None Declared.

\section{PO006}

\section{INTRAOPERATIVE GOAL DIRECTED HEMODYNAMIC THERAPY IN NONCARDIAC SURGERY: A SYSTEMATIC REVIEW AND META ANALYSIS}

Javier Ripollés Melchor ${ }^{1}$, Ángel Espinosa ${ }^{2}$, Rubén Casans Francés ${ }^{3}$, Eugenio Martínez Hurtado ${ }^{1}$, Alberto Gutiérrez Martínez ${ }^{1}$, Alfredo Abad Gurumeta ${ }^{4}$, José Manuel Ramírez ${ }^{*}$ on behalf of GERM, José María Calvo Vecino ${ }^{1}$ on behalf of GERM on behalf of EAR Group. Evidence Anesthesia Review

${ }^{1}$ Anesthesia and Critical Care, Hospital Universitario Infanta Leonor, Madrid, Spain, ${ }^{2}$ Blekinge County Council Hospital, Kalskrona, Sweden, ${ }^{3}$ Anesthesia and Critical Care, Hospital Universitario Lozano Blesa, Zaragoza, ${ }^{4}$ Anesthesia and Critical Care, Hospital Universitario La Paz, Madrid, ${ }^{5}$ General Surgery, Hospital Universitario Lozano Blesa, Zaragoza, Spain

Objectives: The Goal directed hemodynamic therapy (GDHT) is an approach focused on the use of cardiac output (CO) and related parameters as end-points for fluids and drugs to optimize tissue perfusion and oxygen delivery. Primary aim: To determine the effects of intraoperative GDHT on postoperative complications rates.

Methods: Meta-analysis of the effects of GDHT in adult noncardiac surgery on postoperative complications and mortality using PRISMA methodology. A systematic search was performed in Medline PubMed, Embase, and the Cochrane Library (last update, October 2014). Inclusion criteria: Randomized clinical trials (RCTs) in which intraoperative GDHT was compared to conventional fluid management in noncardiac surgery. Exclusion criteria: Trauma and pediatric surgery studies and that using pulmonary artery catheter. End-points: Postoperative complications (primary) and mortality (secondary). Those studies that fulfilled the entry criteria were examined in full and subjected to quantifiable analysis, pre-defined subgroup analysis (stratified by type of monitor, therapy, and hemodynamic goal), and pre-defined sensitivity analysis.

Results: 51 RCTs were initially identified, 24 fulfilling the inclusion criteria. 5 RCTs were added by manual search, resulting in 29 RCTs in the final analysis, including 2,654 patients. A significant reduction in complications for GDHT was observed (RR: 0.70, 95\% CI: $0.62-$ $0.79, \mathrm{p}<0.001)$. No significant decrease in mortality was achieved (RR: $0.76,95 \%$ CI: $0.45-1.28, p=0.30$ ). Quality sensitive analyses confirmed the main overall results.

Conclusion: Intraoperative GDHT with minimally invasive monitoring decreases postoperative complications in no cardiac surgery, although was not able to show a significant decrease in mortality rate.

Disclosure of Interest: None Declared. 


\section{PO007}

\section{FACTORS PREDICTING 30 DAY READMISSION AFTER LAPAROSCOPIC COLORECTAL CANCER SURGERY WITHIN AN ENHANCED RECOVERY PROGRAMME}

Nader Francis* ${ }^{1}$, Jennifer Mason ${ }^{1}$, Emad Salib ${ }^{2}$, Lucy Allenby ${ }^{1}$, David Messenger ${ }^{1}$, Andrew Allison ${ }^{1}$, Neil Smart ${ }^{3}$, Jonathan Ockrim ${ }^{1}$

${ }^{1}$ Surgery, Yeovil Hospital NHS Foundation Trust, Yeovil, ${ }^{2}$ University of Liverpool, Liverpool, ${ }^{3}$ Surgery, Royal Devon \&Exeter NHS Foundation Trust, Exeter, United Kingdom

Objectives: Hospital readmission within 30 days of surgery has become a marker of poor quality patient care. This study aimed to investigate factors predictive of 30 day readmission after laparoscopic colorectal cancer surgery within an Enhanced Recovery After Surgery (ERAS) programme.

Methods: Consecutive patients undergoing laparoscopic surgery for colorectal cancer within an ERAS programme between 2002 and 2009 were included. Data were collected relating to patient demographic, neoadjuvant chemoradiotherapy, ERAS compliance, operative and post-operative outcomes. A logistic regression model was used to identify factors associated with readmissions, after adjusting for potential effect of co-variables simultaneously.

Results: A total of 268 cancer patients underwent laparoscopic colorectal surgery (108 rectal resections), of which 34 patients were readmitted $(12.5 \%)$. Bowel obstruction [29\%] and surgical site infection [18\%] were the most frequent reasons for readmission. The use of neoadjuvant therapy [Odds Ratio $=4.49$ (95\% Confidence Interval 1.41-14.3); $\mathrm{p}=0.011]$ and ERAS compliance above $93 \%$ $[\mathrm{OR}=0.38(95 \%$ CI $0.18-0.84) ; \mathrm{p}=0.016]$ were independent predictors of readmission.

Conclusion: Poor ERAS compliance and preoperative chemoradiotherapy were significant predictors of readmission following laparoscopic colorectal cancer surgery. Further research is required to expand the scope of ERAS beyond hospital discharge.

Disclosure of Interest: None Declared.

\section{PO008}

\section{COST-BENEFIT ANALYSIS OF THE IMPLEMENTATION OF AN ENHANCED RECOVERY PROTOCOL IN LIVER SURGERY}

Gaëtan-Romain Joliat ${ }^{*}{ }^{1}$, Ismaïl Labgaa ${ }^{1}$, Martin Hübner ${ }^{1}$, Anne-Claude Griesser ${ }^{2}$, Markus Schäfer ${ }^{1}$, Nicolas Demartines ${ }^{1}$

${ }^{1}$ Visceral Surgery, ${ }^{2}$ Medical Direction, University of Lausanne Hospital, Lausanne, Switzerland

Objectives: Enhanced recovery (ERAS) programs after surgery have shown to facilitate the postoperative recovery and to improve clinical outcomes for various types of surgery (1-3). Cost-effectiveness of ERAS was demonstrated for colorectal surgery (4). The aim of this study was to analyze the costs of the implementation of an ERAS program for liver surgery.

Methods: A dedicated ERAS protocol for liver surgery was implemented in our department in July 2013. From July 2013 to July 2014, all consecutive patients undergoing liver surgery were treated within this protocol and prospectively documented (ERAS group). They were compared in terms of costs (pre-, intra-, and postoperative costs) with a series of patients before ERAS implementation (pre-ERAS group). ERAS-specific costs included patient logbooks, carbohydrate drinks, costs for the audit system and quarterly meetings, and the salary of the ERAS-dedicated nurse. Cost means per patient were calculated using the bootstrap method and compared with a bootstrap independent T-test. A cost-minimization analysis was then performed. Results: Seventy-four patients in the ERAS group were compared with 100 patients in the pre-ERAS group. There was no statistically significant difference in terms of patient and operation characteristics between the two groups, except for the number of laparoscopy $(\mathrm{n}=18$ in the ERAS group, $\mathrm{n}=9$ in the pre-ERAS group, $p=0.011)$. In the ERAS group, 36 patients had a complication vs. 64 in the pre-ERAS group $(\mathrm{p}=0.046)$. The median length of hospital stay was shorter for the ERAS group ( 8 vs. 10 days, $p=0.006$ ). The total mean costs per patient were 33,053 euros and 36,241 euros for ERAS and pre-ERAS, respectively $(\mathrm{p}=0.042)$. The specific costs of ERAS were estimated to be 550 euros per patient. The costminimization analysis showed a total mean difference of costs per patient of 2,638 euros in favor of the ERAS group $(\mathrm{p}=0.423)$.

Conclusion: ERAS for liver surgery is cost-effective with a gain of 2,638 euros per patient included in the protocol. The cost savings can be partially attributed to a diminution of complications and hospital stay.

Disclosure of Interest: None Declared. 


\section{PO009}

\section{PERIOPERATIVE GOAL-DIRECTED HEMODYNAMIC THERAPY IN NONCARDIAC SURGERY: A SYSTEMATIC REVIEW AND META-ANALYSIS}

Javier Ripollés Melchor ${ }^{1}$, Rubén Casans Francés ${ }^{2}$, Ángel Espinosa ${ }^{3}$, Eugenio Martínez Hurtado ${ }^{1}$, Alberto Gutiérrez Martínez ${ }^{1}$, Alfredo Abad Gurumeta ${ }^{4}$, José Manuel Ramírez ${ }^{*}{ }^{5}$, José María Calvo Vecino ${ }^{1}$ on behalf of EAR Group. Evidence Anesthesia Review

${ }^{1}$ Anesthesia and Critical Care, Hospital Universitario Infanta Leonor, Madrid, ${ }^{2}$ Anesthesia and Critical Care, Hospital Universitario Lozano Blesa, Zaragoza, Spain, ${ }^{3}$ Anesthesia and Critical Care, Blekinge County Council Hospital, Kalskrona, Sweden, ${ }^{4}$ Anesthesia and Critical Care, Hospital Universitario La Paz, Madrid, ${ }^{5}$ General Surgery, Hospital Universitario Lozano Blesa, Zaragoza, Spain

Objectives: Goal-directed fluid therapy (GDHT) has been proposed as a method to reduce complications and mortality.

Methods: Meta-analysis of the effects of perioperative GDHT in adult noncardiac surgery on mortality and postoperative complications using PRISMA methodology. A systematic search was performed in Medline, PubMed, Embase, and the Cochrane Library (last update, October 2014). Inclusion criteria: Randomized clinical trials (RCTs) in which perioperative GDHT was compared to conventional fluid management in noncardiac surgery. Exclusion criteria: Trauma and pediatric surgery studies. Endpoints: mortality and number of patients with complications.

Results: 39 RCTs were initially identified, 8 fulfilling the inclusion criteria. 2 RCTs were added by manual search, resulting in 10 RCTs in the final analysis, including 1,527 patients. Those studies that fulfilled the entry criteria were examined in full and subjected to quantifiable analysis, pre-defined subgroup analysis (stratified by supraphysiological or physiological hemodynamic goal; and by time the intervention was carried out, perioperative or postoperative), and pre-defined sensitivity analysis. Main A significant reduction was observed in mortality associated with GDHT compared to conventional fluid therapy (RR: $0.63,95 \% \mathrm{CI}: 0.42-0.94, \mathrm{p}=0.02$ ). However, no differences were found in the number of patients with complications (RR: $0.75,95 \% \mathrm{CI}: 0.50-1.17, \mathrm{p}=0.21$ ), and the sensitivity analysis did not confirm the results.

Conclusion: This meta-analysis, with its limitations, shows that the use of perioperative GDHT may reduce postoperative mortality, but it is unable to show a reduction in the number of patients with complications.

Disclosure of Interest: None Declared.
PO010

\section{EFFECT OF A 4-WEEK MULTIMODAL PREHABILITATION PROGRAM ON LEAN BODY MASS IN CANCER PATIENTS UNDERGOING COLORECTAL RESECTION: RESULTS FROM A RANDOMIZED CONTROLLED TRIAL}

Sarah-Ève Loiselle ${ }^{1,2}$, Rashami Awasthi ${ }^{2}$, Julia Munden ${ }^{2}$, Celena Scheede-Bergdahl ${ }^{3}$, Alexander Sender Liberman ${ }^{4}$, Francesco Carli ${ }^{*}{ }^{1,2}$

${ }^{1}$ Dietetics and Human Nutrition, McGill University, ${ }^{2}$ Anesthesia, McGill University Health Center, ${ }^{3}$ Kinesiology and Physical Education, McGill University, ${ }^{4}$ Surgery, McGill University Health Center, Montreal, Canada

Objectives: In previous work we showed that a 4-week multimodal prehabilitation program (nutrition, exercise, relaxation) impacts positively on postoperative functional capacity. This study aims to determine the effect of a prehabilitation program initiated 4 weeks before surgery on lean body mass (LBM) in patients undergoing colorectal surgery for cancer, and to compare it with a similar rehabilitation program initiated immediately after surgery.

Methods: Fifty-two colorectal cancer patients were randomized to either prehabilitation (prehab; $\mathrm{n}=27$ ) or rehabilitation (rehab; $\mathrm{n}=25)$. A whey protein supplement (10-20 g/day) and a home-based aerobic and resistance exercise program were given either 4 weeks before surgery (prehab) or immediately after surgery (rehab) to be continued in both groups for 8 weeks post-operatively. Prehab also received one supervised exercise session every week before surgery. LBM, expressed as percent of body weight (\%LBM: LBM (kg)/body weight $\left.(\mathrm{kg})^{*} 100\right)$, was measured using a hand-to-foot bioelectrical impedance analyzer. Data was collected at baseline (BL), the day before surgery (preop), at 4 and 8 weeks post-surgery. Change in \%LBM among groups was tested using Mann-Whitney-Wilcoxon test.

Results: At baseline, groups were not different regarding gender, age $(71 \pm 9$ y), height, weight $(80.6 \pm 12.9 \mathrm{~kg}), \% \mathrm{LBM}(67.4 \pm 8.9 \%)$, percent body fat, functional walking capacity, physical activity and nutritional status. Median change in \%LBM from baseline to preop was $1.01 \%$ (IQR 0.38 to 2.58 ) for prehab versus $0.03 \%$ (IQR -0.26 to 1.62) for rehab $(\mathrm{p}=0.02)$. Overall, the prehab group achieved a greater median increase in \%LBM compared to rehab $(2.17 \%$ (IQR 0.92 to 4.36 ) versus $0.17 \%$ (IQR -0.29 to 1.74 ), respectively, $\mathrm{p}<0.01)$.

Conclusion: A multimodal program initiated 4 weeks before surgery improved \%LBM more than a similar program commenced immediately after surgery, suggesting that prehabilitation could positively affect body composition pre- and post-surgery, and therefore impact functional recovery.

Disclosure of Interest: None Declared. 


\section{PO011}

\section{CAN ENHANCED RECOVERY AFTER LAPAROSCOPIC COLORECTAL CANCER SURGERY IMPACT ON LONG TERM SURVIVAL?}

Nader Francis ${ }^{1}$, Laura Fraser* ${ }^{*}$, Emad Salib ${ }^{2}$, Lucy Allenby ${ }^{3}$, David Messenger $^{3}$, Andrew Allison ${ }^{3}$, Neil Smart ${ }^{4}$, Jonathan Ockrim ${ }^{3}$

${ }^{1}$ Surgery, Yeovil Hospital NHS Foundation Trust, Yeovil, ${ }^{2}$ University of Liverpool, Liverpool, ${ }^{3}$ Surgery, Yeovil Hospital NHS Foundation Trust, Yeovil, ${ }^{4}$ Surgery, Royal Devon \&Exeter NHS Foundation Trust, Exeter, United Kingdom

Objectives: The impact of Enhanced Recovery After Surgery (ERAS) and laparoscopic techniques for colorectal cancer on short term post-operative outcomes has been widely studied confirming its benefits to reduce stress response, length of hospital stay and postoperative complications. However, there has been little research into the long term impact of ERAS in terms of survival. This study aimed to investigate whether the combination of ERAS protocol and laparoscopic techniques for colorectal cancer impacts on disease free survival (DFS) and overall survival (OS) at 12 years and also to examine the factors that can predict survival.

Methods: Data was collected retrospectively on patients who received laparoscopic surgical treatment for colorectal cancer at Yeovil District Hospital NHS Foundation Trust between 2002 and 2009. DFS and OS were calculated using Kaplan-Meier methods. Cox regression model was used to evaluate the factors that predict survival. Statistical analysis of prognostic factors was carried out using SPSS statistical software.

Results: A total of 268 colorectal cancer patients who underwent laparoscopic surgery were included in the study. The median age was 70.5 years of which 139 patients were men (51.9\%). 203 patients (75.7\%) successfully followed ERAS protocol (set as over $80 \%$ compliance), with a median compliance with ERAS elements of $93 \%$. Long term survival showed mean DFS was $70.9 \%$ and OS was $74.3 \%$ at 11 years. Using Cox regression analysis, ERAS compliance was found to be significant for both DFS $(p=0.042)$ and OS $(p=0.005)$. However, in the final Cox regression model, only age $(\mathrm{p}=0.013)$, disease stage $(p=0.0003)$ and American Society of Anaesthesiologists (ASA) grade $(\mathrm{p}=0.03)$ were predictors of OS and age $(p=0.006)$, operative time $(p=0.001)$ and preoperative albumin $(\mathrm{p}=0.04)$ remained as significant predictors of DFS

Conclusion: Adoption of ERAS protocol after laparoscopic techniques for colorectal cancer resection may result in a favourable long term survival. Modifiable factors that significantly predict long term survival are operative time and preoperative albumin

\section{PO012}

\section{THE GREENIE BOARD: NAVAL INNOVATION TO IMPROVE INTRAOPERATIVE COMPLIANCE WITHIN OUR ERAS PROTOCOL}

Yvette Console $^{*}{ }^{1}$, Alexander Smirk ${ }^{1}$, Jonathan Nicholson ${ }^{1}$, Nicole Hunt ${ }^{1}$, Bernhard Riedel ${ }^{1}$

${ }^{1}$ Department of Anaesthesia \& Pain Medicine, Peter MacCallum Cancer Centre, Melbourne, Australia

Objectives: Despite the success of an ERAS bundle of care, many institutions have found that after implementing ERAS, with time compliance wanes, resulting in loss of any long-term benefits (1). Ongoing audit and personalised feedback methods have been reported to provide a basis for producing progressive improvement in compliance and clinical care (2). One such method of a continuous personalised feedback system is the 'Greenie Board' utilised by the USA navy to evaluate and improve aircraft carrier flight landings. Our objective is to implement and identify whether using the continuous personalised feedback system, the Greenie Board can improve compliance with anaesthesia components of our ERAS protocol.

Methods: Continuous and prospective clinical audit of ERAS anaesthesia components was compared in surgical patients allocated to the ERAS protocol. We compared protocol compliance 6-months before (Jan-Jun) and 6-months after (Jul-Dec) the introduction of the Greenie Board Feedback system. Anaesthetic records for all ERAS cases were reviewed and scored out of 7 (1 point for each of the 7 identified separate mandatory anaesthesia ERAS protocol components). A heat map was then created using a colour-coded system (6-7 points $=$ Green $; 5$ points $=$ Yellow; 4 points $=$ Amber $;<4$ points $=$ Red) and displayed on the anonymous Greenie Board within the operating room suite.

Results: 194 consecutive ERAS patients were included in the audit. Green cases improved from $33 \%$ to $74 \%$ of patients ( $p<0.0001$ ). Largest improvements were seen in the areas of antibiotic re-dosing ( $73 \%$ to $92 \%$ p0.004), use of appropriate anti-emetic prophylaxis (49\% to $70 \%$ p0.002), use of Goal Directed Therapy (69\% to $87 \%$ p0.006), and appropriate postoperative fluid restriction (27\% to $71 \%$ $\mathrm{p}<0.0001)$.

Conclusion: Implementing the ERAS Greenie Board in our department resulted in significantly improved adherence to basic anaesthetic components of our ERAS protocol. Further research is recommended to evaluate if this improvement in protocol adherence results in better patient outcomes.

Disclosure of Interest: None Declared.

Disclosure of Interest: None Declared. 


\section{PO013}

\section{RISK FACTORS AND SURVIVAL AFTER UPPER ABDOMINAL SURGERY. A PROSPECTIVE COHORT STUDY}

Eirik K. Aahlin ${ }^{*}{ }^{1}$, Gerd Tran $\varnothing^{2}$, Neil Johns ${ }^{3}$, Arild Horn ${ }^{4}$, Jon Arne Søreide ${ }^{5}$, Kenneth Fearon ${ }^{6}$, Arthur Revhaug ${ }^{1}$, Kristoffer Lassen ${ }^{1}$

${ }^{1}$ Department of GI and HPB Surgery, University Hospital of Northern Norway, Tromsoe, ${ }^{2}$ Department of Gastrointestinal Surgery, St. Olavs Hospital - Trondheim University Hospital, Trondheim, Norway, ${ }^{3}$ Clinical Surgery, University of Edinburgh, Royal Infirmary of Edinburgh, Edinburgh, United Kingdom, ${ }^{4}$ Dept. of Abdominal and Emergency Surgery, Haukeland University Hospital, Bergen, ${ }^{5}$ Department of Gastrointestinal Surgery, Stavanger University Hospital, Stavanger, ${ }^{6}$ Clinical Surgery, University of Edinburgh, Royal Infirmary of Edinburgh, Edinburgh, Norway

Objectives: Preoperative weight loss and abnormal serum-albumin have traditionally been associated with reduced survival [1]. More recently, the significance of the relative proportion of skeletal muscle, visceral and subcutaneous adipose tissue has been examined with conflicting results [2]. We aimed to investigate how preoperative body composition relates to overall survival after upper abdominal surgery and compare this to established predictors.

Methods: From 2001 to 2006, 447 patients were included in a Norwegian multicenter randomized controlled trial investigating food at will after major upper abdominal surgery [3]. Patients were now, six years later, analyzed as a single prospective cohort and overall survival was retrieved from the National Population Registry. Body composition indices were calculated from CT images taken within three months preoperatively.

Results: Preoperative serum-albumin $<35 \mathrm{~g} / 1 \quad(\mathrm{HR}=1.80$, $\mathrm{p}<0.001)$ and weight loss $>5 \%(\mathrm{HR}=1.48, \mathrm{p}=0.005)$ were independently associated with reduced survival. There was no association between any of the preoperative body composition indices and reduced survival.

Conclusion: Our study has confirmed the robust significance of the traditional indicators preoperative serum-albumin and weight loss. The body composition indices did not prove beneficial as global tools for risk assessment in upper abdominal surgery.

Disclosure of Interest: None Declared.

\section{PO014}

\section{A RANDOMISED FEASIBILITY STUDY TO ASSESS A NOVEL STRATEGY TO RATIONALISE FLUID IN PATIENTS FOLLOWING CARDIAC SURGERY}

Rachael Parke* 1, 2, 3, Shay McGuinness ${ }^{1,2,3}$, Eileen Gilder ${ }^{1}$, Lianne McCarthy ${ }^{1}$, Keri-Anne Cowdrey ${ }^{1}$

${ }^{1}$ Cardiothoracic and Vascular Intensive Care Unit, Auckland City Hospital, Auckland, ${ }^{2}$ Medical Research Institute of New Zealand, Wellington, New Zealand, ${ }^{3}$ Australian and New Zealand Intensive Care Research Centre, Department of Epidemiology and Preventive Medicine, Monash University, Melbourne, Australia

Objectives: Following cardiac surgery, patients receive large amounts of fluid in the Intensive Care Unit (ICU). Fluid volume strategies have been investigated in other ICU populations such as those with acute respiratory distress syndrome or sepsis however widespread practice variation exists. To date there have been no reported studies of peri-operative fluid management strategies in patients undergoing cardiac surgery. We plan to conduct a multicentre randomised controlled trial of a conservative fluid regime involving a novel use of advanced hemodynamic monitoring in patients following cardiac surgery, and report here the results of a feasibility study that evaluated the efficacy and safety of the proposed regime. This study aimed to test the hypothesis that a stroke volume variationbased algorithm would reduce the amount of intravenous fluid administered to patients after cardiac surgery.

Methods: Following ethical approval a single-centre, prospectively randomised interventional study was undertaken. Participants were randomised to either usual care or to a protocolised algorithm utilising stroke volume variation to restrict fluid administration to patients who were deemed to have an inadequate cardiac output and who were likely to be volume responsive. The study protocol lasted from ICU admission to de-sedation or 24 hours, whichever occurred first.

Results: We randomised 144 patients over 9 months. Less bolus fluid and less total overall fluid volume was administered in the intervention group as compared to the usual care group from ICU admission to extubation (median (IQR) $1625 \mathrm{mls} \mathrm{(500-3406)} \mathrm{vs.} 2525 \mathrm{mls}$ $(1438-5250) \mathrm{p}<0.001)$ and $2054 \mathrm{mls}(912-4276)$ vs. $2979 \mathrm{mls}$ (2070-6581), $\mathrm{p}=0.001$ respectively). There was no significant difference in incidence of acute kidney injury or the average amount of fluid administered to the usual care group at the beginning compared the end of the study.

Conclusion: This study demonstrates that it is both possible and safe to achieve a significant reduction in the amount of fluid administered to patients allocated to the conservative fluid protocol. These results suggest that a planned multi-centre study is both justified and feasible.

Disclosure of Interest: R. Parke Grant / Research support from: This work was supported by: the Health Research Council of New Zealand by way of a Feasibility Study award (HRC13/756) (RLP). Research in the CVICU is supported in part by an unrestricted grant from Fisher and Paykel Healthcare, New Zealand. Edwards Lifesciences provided the consumables for this study without charge and loaned the monitors for the life of the study. These sponsors had no input into the study design and no access to trial data. All analyses, reporting and decisions to publish have been made independent of the sponsors., S. McGuinness Grant / Research support from: Received research support and speaking fees for Edwards Lifescience and Baxter, E. Gilder: None Declared, L. McCarthy: None Declared, K.-A. Cowdrey: None Declared. 


\section{PO015}

\section{IMPLEMENTATION OF SYSTEMATIC COLORECTAL ENHANCED RECOVERY AFTER SURGERY PROGRAM (ERAS) HAS AN IMPACT ON THE OUTCOME IN NON ERAS PATIENTS AFTER LIVER RESECTION}

Ismail Labgaa $^{*}{ }^{1}$, Ghada Jarrar ${ }^{1}$, Gaëtan-Romain Joliat ${ }^{1}$, Pierre Allemann ${ }^{1}$, Sylvain Gander ${ }^{2}$, Catherine Blanc ${ }^{3}$, Martin Hubner ${ }^{1}$, Nicolas Demartines ${ }^{1}$

${ }^{1}$ Department of Visceral Surgery, University Hospital of Lausanne (CHUV), Lausanne, ${ }^{2}$ Department of Anaesthesiology, Hospital of Yverdon, Yverdon, ${ }^{3}$ Department of Anaesthesiology, University Hospital of Lausanne (CHUV), Lausanne, Switzerland

Objectives: Enhanced recovery pathways like ERAS have become standard of care in colorectal surgery. Recently, ERAS principles were extended to liver surgery. However, assessing the impact of ERAS in liver surgery via prospective randomized studies is very difficult. Indeed, ERAS colorectal patients may influence the behavior of liver patients in the same ward. In other words, comparing liver patients with or without ERAS protocol may be biased because the "control" group may be influenced by previous ERAS implementation. This study aims to analyze the collateral impact of an ERAScolorectal pathway on liver resections.

Methods: This retrospective study compared 3 consecutive cohorts of patients undergoing elective liver surgery: before any ERAS implementation (pre ERAS $n=50$ ), after colorectal ERAS implementation (intermediate $\mathrm{n}=50$ ), and after liver ERAS implementation (ERASliver $\mathrm{n}=74$ ). The study period was from June 2010 until July 2013. Compliance with the ERAS protocol was assessed in the 3 groups for the 3 phases of care (pre-, peri- and post-operative). Outcomes were functional recovery, complications, hospital stay, and readmissions within 30days after surgery.

Results: The 3 compared groups were similar for demographics; laparoscopy was more frequent in ERAS-liver patients $(\mathrm{p}=0.009)$. Preoperative compliance was enhanced by both implementations $(4.5 \%, 32.2 \%, 81.1 \%, \mathrm{p}<0.001 /<0.001)$. Perioperative and postoperative compliance rates also increased after both implementations, with no significant difference ( $p=0147 / 0.069$ ). As a result, ERASliver group displayed the highest compliance, with overall compliance of $73.8 \%$, compared to $39.9 \%$ and $57.4 \%$ for pre-ERAS and Intermediate groups $(\mathrm{p}=0.072 / 0.056)$. Comprehensive Complication Index showed a trend for reduced complications in ERAS-liver group $(20.9,20.9,8.7, \mathrm{p}=0.185)$. Hospital stay was reduced by more than two days $(10.5,8.5,8, \mathrm{p}=0.005)$ without increased readmissions $(\mathrm{p}=0.158)$.

Conclusion: These results suggest that the previous implementation of ERAS for colorectal surgery already led to a collateral impact on liver surgery. Moreover, these results strongly suggest that ERASliver pathway is applicable and beneficial in liver surgery.

Disclosure of Interest: None Declared.
PO016

\section{A MULTI-INSTITUTIONAL CONTROLLED STUDY ON SAFETY AND EFFICACY OF AN ENHANCED RECOVERY AFTER SURGERY (ERAS) PROTOCOL TO PATIENTS WITH COLON CANCER}

Hirofumi Ota ${ }^{*}{ }^{1}$, Masakazu Ikenaga ${ }^{2}$, Shu Okamura ${ }^{3}$, Yasuhiro Miyake $^{4}$, Yujiro Fujie ${ }^{5}$, Hirotoshi Kin ${ }^{6}$, Kimimasa Ikeda ${ }^{7}$, Ichro Takemasa $^{8}$, Masataka Ikeda ${ }^{9}$, Kohei Murata ${ }^{3}$, Junichi Hasagawa ${ }^{2}$, Tsunekazu Mizushima ${ }^{8}$, Hirofumi Yamamoto ${ }^{8}$, Mitsugu Sekimoto ${ }^{9}$, Riichiro $\mathrm{Nezu}^{4}$, Yuichiro Doki ${ }^{8}$, Masaki Mori ${ }^{8}$ on behalf of Clinical Study Group of Osaka University (CSGO), Colorectal Group on behalf of Clinical Study Group of Osaka University (CSGO), Colorectal Group

${ }^{1}$ Department of Digestive Surgery, Ikeda City Hospital, ${ }^{2}$ Department of Surgery, Osaka Rosai Hospital, ${ }^{3}$ Department of Surgery, Suita Municipal Hospital, ${ }^{4}$ Department of Surgery, Nishinomiya Municipal Hospital, ${ }^{5}$ Department of Surgery, NTT West Japan Hospital, ${ }^{6}$ Department of Surgery, Rinku General Medical Center, ${ }^{7}$ Department of surgery, Mino City Hospital, ${ }^{8}$ Department of Digestive Surgery, Osaka University Graduate School of Medicine, ${ }^{9}$ Department of Surgery, National Hospital Organization, Osaka National Hospital, Osaka, Japan

Objectives: The aim of this study was to evaluate safety and efficacy of an ERAS protocol in a multi-institutional controlled study.

Methods: Three hundred and twenty patients with ASA grade I or II were registered in this study. They underwent elective open or laparoscopic colonic resection or high anterior resection between April 2011 and January 2014 at six institutions, among which three hospitals implemented ERAS protocol (ERAS group, $n=159$ ), and the other three executed conventional cares (conventional group, $\mathrm{n}=161$ ). The primary outcome measures were surgical complications (surgical site infection, ileus, anastomotic leakage, intraperitoneal or anastomotic bleeding). Secondary outcome measures were non-surgical complications, length of hospital stay, mortality or re-admission rate within 30 days after surgery, and day on which the first flatus or stool was recognized, food intake started, or intravenous fluids discontinued.

Results: Most surgeries were performed laparoscopically in each group (ERAS group vs. conventional care group: $96.9 \%$ vs. $91.3 \%$ ). An incidence of surgical complications was $17.6 \%$ in the ERAS group versus $17.4 \%$ in the conventional care group, showing no statistically significant difference $(\mathrm{p}=0.842)$. A few non-surgical complications were recognized in conventional care group alone (respiratory disorders: $0.6 \%$, cardiovascular ones: $0.6 \%$, or hepatorenal ones: $0.6 \%$, delirium 1.9\%). Earlier oral food intake and discontinuation of intravenous fluids were implemented in the ERAS group (ERAS group vs conventional care group: median (range) 1 (1-31) vs. 3 (1-9) day (s); p $<0.001,1$ (1-11) vs. 5 (3-35) day (s); p $<0.001$, respectively). The first passage of flatus or stool occurred by one day earlier in the ERAS group (1 (1-5) vs. 2 (1-5) day (s); p < 0.001, 2 (1-6) vs. 3 (1-7) day (s); $<<0.001$, respectively). The median length of hospital stay was reduced by 5.5 days in the ERAS group $(\mathrm{p}<0.001)$. No mortality was seen in each group. A readmission rate was $1.3 \%$ in ERAS group vs. $0 \%$ in conventional care group $(\mathrm{p}=0.153)$.

Conclusion: In conclusion safety and efficacy on ERAS protocol was shown in a multi-institutional controlled study in Japan.

Disclosure of Interest: None Declared. 


\section{PO017}

\section{"THE LEARNING CURVE OF INTRODUCING A SUCCESSFUL ERAS PROGRAMME FOR ROBOTIC RADICAL CYSTECTOMY"}

Chris Jones $^{*}{ }^{1,2}$, Grigoris Athanasiadis ${ }^{3}$, Matthew Dickinson ${ }^{1,2}$, Michael Swinn ${ }^{3}$, Matthew Perry ${ }^{4}$, Krishna Patil ${ }^{3}$

${ }^{1}$ Anaesthesia, Royal Surrey County Hospital NHS Foundation Trust, ${ }^{2}$ SPACeR, Surrey Perioperative Anaesthesia and Critical Care Collaborative Research Group, ${ }^{3}$ Urology, Royal Surrey County Hospital NHS Foundation Trust, Guildford, ${ }^{4}$ Urology, St Georges Hospital, London, United Kingdom

Objectives: Radical cystectomy is the standard of care for patients with muscle-invasive bladder cancer. However this operation carries significant morbidity, mortality and a long hospital stay ((LoS) median 13 days in UK). We describe the implementation of an ERAS programme for a new regional cystectomy service in the UK.

Methods: An ERAS programme was devised using the following elements: patient education, pre-operative carbohydrate drinks, minimally invasive (robotic-assisted) surgery, spinal analgesia, goaldirected fluid therapy, early feeding (with nutritional supplementation) and intensive early mobilisation. This was a new service with surgeons having mentoring help from an experienced surgeon from a nearby centre. We describe two patient cohorts: 13 patients undergoing surgery in 2013 and 22 patients in 2014.

Results: There was no difference in the demographics of the two groups. Operating time and blood loss were both significantly reduced in the two cohorts (see Table 1). The median LoS was significantly reduced in the 2014 cohort when compared to the 2013 ( 5 days [4-15] versus 8 [6-29]). All patients underwent urinary diversion with ileal conduit, except for 3 (in the 2014 cohort) who had orthotopic neobladder procedures. There was one mortality in the 2013 cohort. Morbidity was $15 \%$ in the 2013 cohort (pelvic haematoma (ClavienDindo Classification -3a) and bowel obstruction (3b)) and $9 \%$ in the 2014 cohort (AKI (2) and DVT (2)). There was only one readmission in each cohort.

Table 1 Demographics, operating time, blood loss and Hospital LoS between cohorts

\begin{tabular}{llll}
\hline & $\begin{array}{l}2013 \text { cohort } \\
(\mathrm{n}=13)\end{array}$ & $\begin{array}{l}\text { 2014 cohort } \\
(\mathrm{n}=22)\end{array}$ & $\mathrm{p}$-value \\
\hline Age (mean-years) & 72.9 & 71.6 & 0.632 \\
$\begin{array}{l}\text { ASA (median) } \\
\begin{array}{c}\text { Operation Time } \\
\text { (mean - mins) }\end{array}\end{array}$ & 2 & 2 & 0.087 \\
$\begin{array}{c}\text { Blood loss } \\
\quad \text { (mean - mls) }\end{array}$ & 269 & 368 & $\mathbf{0 . 0 4 4}$ \\
$\begin{array}{c}\text { Hospital LoS } \\
\text { (median - days) }\end{array}$ & 8 & 177 & $\mathbf{0 . 0 2 8}$ \\
\hline
\end{tabular}

Conclusion: With close mentoring it shows that operating time and blood loss can be quickly reduced and together with a comprehensive ERAS programme, short LoS's can be achieved.

Disclosure of Interest: None Declared.

\section{PO018}

\section{PATIENTS WITH LOW BASELINE FUNCTIONAL CAPACITY BENEFIT FROM MULTIMODAL PREHABILITATION}

Rashami Awasthi ${ }^{1}$, Chelsia Gillis ${ }^{1}$, Julia Munden ${ }^{1}$, Sarah-Eve Loiselle $^{1}$, Celena Scheede-Bergdahl ${ }^{1,2}$, Francesco Carli ${ }^{*}{ }^{1,2}$

${ }^{1}$ Anesthesia, Montreal General Hospital, ${ }^{2}$ Kinesiology and Physical Education, McGill University, Montreal, Canada

Objectives: The purpose of this study is to determine which patients would benefit from the multimodal prehabilitation.

Methods: This study involved a reanalysis of data arising from a pilot (1) and a randomized trial (2) with the addition of a new randomized controlled study. All three investigations received ethics approval. The primary outcome measure was functional walking capacity measured by the validated Six-Minute Walk Test (6MWT), which was assessed at baseline when patients were admitted to the study and after an average of 4 weeks of multimodal prehabilitation. The program included a 4-week, home-based unsupervised intervention of moderate aerobic and resistance exercises, nutritional counseling with whey protein supplementation (Immunocal), and relaxation exercises. The patients were divided in two groups: group A included those patients whose baseline 6MWT was below $60 \%$ of the predicted value, and group B included patients whose predicted value was above $60 \%$. The change in $6 \mathrm{MWT}$ over the 4 -week prehabilitation period was compared between the two groups.

Results: Data from 106 patients were analyzed. There were 40 patients in group A and 66 in group B. Gender distribution among the two groups and BMI were similar. The average age of group A was $72 \pm 16$ years compared to group B $66 \pm 20$ years $(\mathrm{p}<0.01)$. There were more patients with ASA 3 and 4 in group A $(p<0.05)$. The increase in 6 MWT during the prehabilitation period in group A was $46 \pm 50 \mathrm{~m}$ while in group B was $22 \pm 43 \mathrm{~m}$ ( $\mathrm{p}<0.01)$. The proportion of patients whose 6MWT improved over $20 \mathrm{~m}$ was significantly greater in group A (70\%) compared with group B (43\%) $(\mathrm{p}<0.05)$.

Conclusion: These preliminary data indicated that patients whose predicted $6 \mathrm{MWT}$ is below $60 \%$ at baseline, tend to be older and with ASA of 3 and 4 . When these patients were enrolled in a multimodal prehabilitation program their functional walking capacity increased by an average $45 \mathrm{~m}$.

Disclosure of Interest: None Declared. 


\section{PO019}

\section{WHAT IS THE POTENTIAL EFFECT ON NATIONAL BED CAPACITY IF ERAS WAS APPLIED TO ALL FRACTURED NECK OF FEMUR PATIENTS?}

\author{
Thomas W. Wainwright ${ }^{*}{ }^{1}$, Robert G. Middleton ${ }^{1,2}$ \\ ${ }^{1}$ Orthopaedic Research Institute, Bournemouth University, \\ Bournemouth, ${ }^{2}$ Trauma and Orthopaedics, Poole Hospital NHS \\ Foundation Trust, Poole, United Kingdom
}

Objectives: ERAS has been shown in some UK hospitals to be successful at improving outcomes such as length of stay (LOS), mortality, and discharge destination when applied to fractured neck of femur (FNOF) pathways. The objective of this study was to model the potential effect of applying Enhanced Recovery principles to all FNOF patients across England.

Methods: Dr Foster software was used to interrogate Hospital Episode Statistic data. Nonelective admissions with a primary diagnosis of FNOF from Nov 2013 to Oct 2014 were analysed. Mean LOS for FNOF patients following an ERAS pathway at Poole Hospital was calculated, and compared to the national mean (all acute non-specialist hospitals). Modelling was applied to evaluate the number of bed days that could be saved if ERAS was introduced and practice was changed nationally to achieve the same LOS as at Poole Hospital.

Results: 63,802 FNOF hospital spells were identified and the national mean length of stay (LOS) for patients was 19.3 days. The mean LOS at Poole Hospital over the same time period was 12.3 days ( 983 spells). This was 6.8 days less than the case-mix adjusted expected LOS, and 7 days less than the national mean. If all of the acute non-specialist hospitals achieved the same LOS as Poole Hospital, then 439,733 bed days could be saved in the year.

Conclusion: FNOF is the most frequent emergency surgical pathway seen in most English hospitals. There is considerable variation in LOS between hospitals. The case-mixed adjusted data suggests that this is due to differences in practice and not patient case-mix. In addition to the likely improvement in quality of care for patients as seen in other surgical procedures, if ERAS was introduced to FNOF pathways nationally there would be highly significant economic and capacity benefits. This is of extreme importance at a time when financial pressures and the need for bed capacity have never been greater.

Disclosure of Interest: None Declared.

\section{PO020}

\section{POPULATION-BASED EVALUATION OF ERAS ADOPTION IN THE STATE OF MICHIGAN}

Scott E. Regenbogen ${ }^{*}{ }^{1}$, Lorraine Burgunder-Zdravkovski ${ }^{1}$, Greta Krapohl $^{1}$, Darrell A. Campbell ${ }^{1}$, Michael J. Englesbe ${ }^{1}$

\section{${ }^{1}$ Surgery, University of Michigan, Ann Arbor, United States}

Objectives: To understand obstacles to adoption of ERAS. ERAS is well described in highvolume specialty centers, but its uptake beyond such institutions has been limited. Within a statewide surgical collaborative, we have promoted ERAS in colon surgery for two years, but need better understanding of front-line providers' beliefs, and obstacles to implementation in order to target ongoing dissemination and implementation strategies.

Methods: We conducted an electronic survey of surgeon champions and surgical case review nurses from all 64 hospitals in the Michigan Surgical Quality Collaborative. We evaluated current use of ERAS, beliefs about its effects on perioperative outcomes, and perceived obstacles to adoption. Analysis was descriptive.

Results: We received 100 complete surveys to date (interim response 77\%) from 55 nurses and 33 surgeons representing 55 hospitals (12 did not identify a role or affiliation). Only $11 \%$ of respondents reported full ERAS implementation for colorectal surgery; $8 \%$ reported ERAS use for other operations. However, $42 \%$ reported use of selected ERAS practices without a formal protocol and $34 \%$ reported ERAS protocols in development. An additional 17\% reported active interest in ERAS in their institution. Only 3 respondents indicated their institution was not interested in ERAS. The vast majority believe ERAS improves outcomes, including: return of bowel function $(80 \%)$, pain scores $(78 \%)$, length of stay $(95 \%)$, complication rates $(80 \%)$, readmission $(73 \%)$, patient satisfaction $(88 \%)$, and cost $(84 \%)$. Only a small minority believe ERAS increases renal insufficiency $(3 \%)$, readmission $(7 \%)$, or post-discharge ancillary care costs $(5 \%)$. The most common reported obstacles to ERAS adoption were resistance from surgeon (41\%) or anesthesiologist (28\%), lack of consensus (41\%), time/logistics (66\%), and funding (57\%). Conclusion: Despite wide acceptance of the effectiveness of ERAS and piecemeal adoption of isolated components, there remain few hospitals in Michigan with fully implemented protocols. Wider adoption will require tailored, mentored implementation to surmount perceived resistance from physicians, and provide support to overcome the time, effort and cost required to initiate comprehensive ERAS protocols beyond select institutions.

\section{PO021}

\section{IMPLEMENTATION OF AN ENHANCED RECOVERY PROGRAM FOR PANCREAS HEAD RESECTION IS HIGHLY COST-EFFECTIVE: RESULTS OF A COST-BENEFIT ANALYSIS OF 161 PATIENTS}

Gaëtan-Romain Joliat ${ }^{*}{ }^{1}$, Ismail Labgaa ${ }^{1}$, David Petermann ${ }^{1}$, Martin Hübner ${ }^{1}$, Anne-Claude Griesser ${ }^{2}$, Nicolas Demartines ${ }^{1}$, Markus Schäfer ${ }^{1}$

${ }^{1}$ Visceral Surgery, ${ }^{2}$ Medical Direction, University of Lausanne Hospital, Lausanne, Switzerland

Objectives: Enhanced recovery (ERAS) programs have shown a decrease of complications and length of hospital stay after different types of surgery (1-3). Cost-effectiveness of ERAS programs was demonstrated mainly for colorectal surgery (4), but no data are yet available for pancreas surgery. The present study aimed to assess the economic aspects of an ERAS program for pancreatoduodenectomy (PD).

Methods: ERAS for pancreas surgery was implemented in our division in October 2012. From October 2012 to October 2014 all consecutive PD patients were recorded as ERAS group. They were compared in terms of costs to all PD performed between January 2010 and October 2012 (pre-ERAS group). Preoperative, intraoperative, and postoperative costs were collected for every patient via the hospital administration. They were compared between the two groups using a bootstrap independent T-test. Specific ERAS-related costs (i.e., ERAS database, full-time ERASdedicated nurse, ERAS meetings, carbohydrates drinks, ERAS logbooks) were calculated.

Results: Seventy-four ERAS patients matched well in terms of demographic and surgical details with 87 patients in the pre-ERAS group. Overall complication rate was 68\% (50/74) and $82 \%(71 / 87)$ in the ERAS and pre-ERAS groups, respectively $(\mathrm{p}=0.046)$. Median hospital stay was shorter for the ERAS group ( 15.5 vs. 19 days, $\mathrm{p}=0.029)$.

Specific ERAS-related costs were 922 euros per patient. Mean total costs per patient were 56,083 euros for the ERAS group and 63,821 euros for the pre-ERAS group $(\mathrm{p}=0.273)$. The mean intensive care unit (ICU) and intermediate care costs per patient were 9,139 euros and 13,793 euros for the ERAS and the pre-ERAS groups, respectively (mean difference: $-4,654$ euros, $\mathrm{p}=0.151$ ).

Conclusion: ERAS implementation for PD was cost-effective in our cohort. Savings can be explained by a reduction of postoperative complications and hospital stay. Furthermore, fewer patients in the ERAS group required an ICU stay, and the duration of the ICU stay was shorter.

Disclosure of Interest: None Declared.

\section{PO022}

\section{RANDOMIZED CLINICAL TRIAL OF AN ENHANCED RECOVERY PROTOCOL FOLLOWING PANCREATICODUODENECTOMY}

Kosei Takagi $^{*}{ }^{1}$, Ryuichi Yoshida ${ }^{1}$, Takashi Kuise ${ }^{1}$, Masashi Utsumi $^{1}$, Daisuke Nobuoka ${ }^{1}$, Yuzo Umeda ${ }^{1}$, Susumu Shinoura ${ }^{1}$, Takahito Yagi $^{1}$, Toshiyoshi Fujiwara ${ }^{1}$

${ }^{1}$ Department of Gastroenterological Surgery, Okayama University Graduate School of Medicine, Dentistry, and Pharmaceutical Sciences, Okayama City, Japan

Objectives: Comprehensive guidelines for perioperative care for pancreaticoduodenectomy (PD) are provided from the Enhanced Recovery After Surgery (ERAS) Society [1]. The aim of this study was to investigate the efficiency of an ERAS protocol compared with conventional perioperative management in patients following PD.

Methods: The elements of ERAS protocols according to ERAS guidelines [1] were preoperative factors (counselling, mobilization, immunonutrition, no bowel preparation, fluid and carbohydrate loading), intraoperative factors (fluid restriction, avoiding hypothermia, epidural and intravenous analgesia) and postoperative factors (early enteral tube feeding and oral intake, synbiotics, early mobilization, glycemic control, early removal of urinary catheter and drains at low risk, fluid restriction, anti-thrombotic prophylaxis). Primary endpoint was length of postoperative hospital stays. Secondary endpoints included postoperative clinical outcomes and compliance with components of the ERAS protocol. Results: From June 2014 to December 2014, 19 patients ( 8 female, 11 male, median age 63) who were scheduled to undergo PD were randomized to receive ERAS protocols (ERAS group; $\mathrm{n}=10$ ) or standard care (control group; $\mathrm{n}=9$ ). ERAS group had a shorter hospital stay ( 21.4 vs 34.2 days, $\mathrm{p}=0.041$ ). There were no differences in mortality, postoperative major complications and readmissions. The ERAS procedure shorten the duration of SIRS and led to earlier oral intake, faster gastrointestinal movements and earlier ambulation.

Conclusion: Multimodal ERAS protocols for PD can reduce postoperative hospital stay and not increase morbidity and mortality. The ERAS strategy can be safe and effective in improving postoperative outcomes.

Disclosure of Interest: None Declared. 


\section{PO023}

\section{USING PATIENT-REPORTED OUTCOMES TO MEASURE FUNCTIONAL RECOVERY AFTER THORACIC SURGERY}

Xin Shelley Wang ${ }^{*}{ }^{1}$, Qiuling Shi ${ }^{1}$, David Rice ${ }^{2}$, Ara Vaporciyan ${ }^{2}$, Keyuri Popat ${ }^{3}$, Charles Cleeland ${ }^{1}$

${ }^{1}$ Symptom Research, ${ }^{2}$ Thoracic Surgery, ${ }^{3}$ Anesthesiology \& Perioperative Medicine, MD Anderson Cancer Center, Houston, United States

Objectives: Measuring patient-reported outcomes (PROs) has become increasingly important for assessing quality of care and guiding patient management. However, PROs have yet to be integrated with traditional clinical outcomes (such as length of hospital stay) to evaluate perioperative care. This study aimed to utilize longitudinal PRO assessments to define the postoperative recovery trajectory in patients undergoing thoracic surgery for lung cancer. Methods: Newly diagnosed patients $(\mathrm{N}=60)$ with stage I or II non-small cell lung cancer who underwent either standard open thoracotomy or video-assisted thoracoscopic surgery (VATS) lobectomy reported multiple symptoms from presurgery to 3 months postsurgery using a validated PRO tool, the MD Anderson Symptom Inventory (MDASI). We conducted KaplanMeier analyses to determine when symptoms and functioning returned to presurgical levels and to mild severity levels during recovery.

Results: The most-severe postoperative symptoms were fatigue, pain, shortness of breath, disturbed sleep, and drowsiness. The median time to return to mild symptom severity for these 5 symptoms was shorter than return to baseline severity, with fatigue taking longer. Pain 5 covere more quickly for patints whor standard open 18 days, respectively; $P=022$ ). Functioning measures (the MDASI interference items walking, work, enjoyment of life, and relations with others) recovered significantly more quickly for patients who underwent VATS than for patients who underwent open thoracotomy (all $\mathrm{P}<.01$ ). Patients who had poor preoperative performance status or comorbidities reported higher postoperative symptom burden (all $P<.05$ ).

Conclusion: This study demonstrates that the MDASI is a sensitive tool for detecting differences in functioning by type of thoracic surgery, preoperative performance status, and comorbid conditions. Our results warrant further evaluation of routine measurement of postoperative symptoms and functioning from the patient's perspective as one effective strategy for evaluating functional recovery during perioperative care.

Disclosure of Interest: None Declared.

\section{PO024}

\section{INSTITUTING PERI-OPERATIVE FLUID GUIDELINES A QUALITY IMPROVEMENT PROJECT}

Sanjiv Patel ${ }^{*}{ }^{1,2}$, Kirstie McPherson McPherson ${ }^{1}$, Matthew Higham ${ }^{1}$, Monty Mythen ${ }^{2}$

${ }^{1}$ Anaesthesia, University College London Hospital, ${ }^{2}$ Anaesthesia, Centre of Anaesthesia, London, United Kingdom

Objectives: Fluid and haemodynamic management is a central to perioperative patient care and has a significant impact on postoperative morbidity and length of hospital stay [1]. Studies including a recent Cochrane review have brought clarity to the need for balanced salt solutions and recent trials have led to the removal of starches form the operating theatre in the United Kingdom [2]. Goal-directed fluid therapy (GDFT) has been shown to benefit patients, specifically those in high-risk groups. Adherence to GDFT is often neglected and formal stratification of risk is frequently omitted. Dat from our own institution shows a clear lack of translation of these recommendations

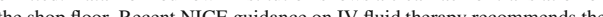
the shop floor. Recent NICE guidance on IV flid therapy recomments considered prescribing of fluids and facilitate translating research into clinical practise [3]

Methods: A formal literature search was performed using Medline, EMBASE and Cochrane review. This evidence was then distributed to stakeholders and a formal round table review was undertaken to produced local guidance.

Results: The algorithm requires patients to be risk stratified utilising tools such as P-POSUM o UK-SORT. Peri-operative fluid guidance was provided for low, intermediate and high risk groups including a goal directed fluid therapy algorithm. A survey of anaesthetists within UCLH indicated that the guidelines have enhanced perioperative fluid prescribing.

Conclusion: Development of this algorithm at UCLH has assisted clinicians in perioperative fluid prescribing. It has helped drive quality in care we and reduce postoperative complications. The guidelines are a working model of the recommendations made in the GIFTASUP guidelines, centred around recommendations made by the Enhanced Recovery after Surgery Society (ERAS).

Disclosure of Interest: None Declared.
PO025

\section{COMPARATIVE EFFECTIVENESS AND ANALYSIS OF POSTOPERATIVE OUTCOMES AFTER ENHANCED RECOVERY PROGRAM FOR ESOPHAGECTOMY}

Francesco Puccetti ${ }^{*}{ }^{1}$, Uberto Fumagalli Romario ${ }^{1}$, Matteo Porta ${ }^{1}$, Alessandra Melis ${ }^{2}$, Stefano de Pascale ${ }^{1}$, Riccardo Rosati ${ }^{2}$

${ }^{1}$ Esophago-gastric Surgery, IRCCS Humanitas, Rozzano (MI), ${ }^{2}$ Gastrointestinal Surgery, IRCCS San Raffaele Hospital, Milan, Italy

Objectives: The aim is to compare the post-operative outcomes after esophagectomy of patients following two clinical pathways: enhanced recovery after surgery (ERAS) criteria based group and previous standard program group. Results deriving from ERAS criteria were also analyzed to identify possible predicting factors.

Methods: Data of a group of consecutive patients with cancer of the esophagus or cardia who underwent esophagectomy with gastric pull up from March 2012 to March 2014 were prospectively collected. They were treated according to a care plan based on ERAS criteria (ERAS-pro group). Results were compared to those obtained in a group of consecutive patients who underwent the same procedures from March 2008 to March 2011 treated according to a standard care plan (OLD-pro group). The results of the 2 groups were compared in terms of morbidity and mortality rate also postoperative length of stay. Compliance to each ERAS item was considered and univariate analysis performed to evaluate its association with ERAS-pro group's morbidity.

Results: 90 patients entered the ERAS-pro group and 91 from the OLD-pro group. The 2 groups were homogeneous regarding their characteristics. There were no significant differences in terms of morbidity $(24.4 \%$ and $36.3 \%$ respectively) and mortality rates $(2.2 \%$ and $6.6 \%$ respectively). The postoperative length of stay was shorter in the ERAS-pro group (10 and 12 days respectively) but not statistically significant. The compliance of patients to the single items of the ERAS program was 60$97 \%$ while statistics analysis reported that only the use of epidural anaesthesia showed a significant association with a higher rate of postoperative complications.

Conclusion: Patients undergoing esophagectomy and treated with an ERAS perioperative care plan have a shorter length of stay, reduced mortality rate with lower postoperative morbidity. Epidura anaesthesia was significantly associated with postoperative complication rate but its role is controversial. Other ERAS items did not show significant associations to morbidity. A protracted evaluation on a larger number of patients is warranted; data on quality of life, and disease free survival should be part of the evaluation.

Disclosure of Interest: None Declared.

\section{PO026}

\section{POSTOPERATIVE HEALTH-RELATED QUALITY OF LIFE PREDICTS OVERALL SURVIVAL AFTER MAJOR UPPER ABDOMINAL SURGERY. A PROSPECTIVE COHORT} STUDY

Eirik K. Aahlin ${ }^{*}{ }^{1}$, Gerd Tran $\varnothing^{2}$, Neil Johns ${ }^{3}$, Arild Horn ${ }^{4}$, Jon Arne Søreide ${ }^{5}$, Kenneth Fearon ${ }^{6}$, Arthur Revhaug ${ }^{1}$, Kristoffer Lassen ${ }^{1}$

${ }^{1}$ Department of GI and HPB surgery, University hospital of Northern Norway, Tromsoe, ${ }^{2}$ Department of Gastrointestinal Surgery, St. Olavs Hospital - Trondheim University Hospital, Trondheim, Norway, ${ }^{3}$ Clinical Surgery, University of Edinburgh, Royal Infirmary of Edinburgh, Edinburgh, United Kingdom, ${ }^{4}$ Dept. of Abdominal and Emergency Surgery, Haukeland University Hospital, Bergen, ${ }^{5}$ Department of Gastrointestinal Surgery, Stavanger University Hospital, Stavanger, ${ }^{6}$ Clinical Surgery, University of Edinburgh, Royal Infirmary of Edinburgh, Edinburgh, Norway

Objectives: Major upper abdominal surgery is often associated with reduced health-related quality of life (HRQOL) and reduced survival [1]. Patients with upper abdominal malignancies often suffer from cachexia, represented by preoperative weight loss and sarcopenia (reduced amount of skeletal muscle) and this might affect both HRQOL and survival. We aimed to investigate how HRQOL is affected by cachexia and how HRQOL relates to long-term survival after major upper abdominal surgery. Methods: From 2001 to 2006, 447 patients were included in a Norwegian multicenter randomized controlled trial investigating food at will after major upper abdominal surgery [2]. In the presen study - six years later, these patients were analyzed as a single prospective cohort and survival data were retrieved from the National Population Registry. Cachexia was derived from data on patientreported preoperative weight loss and sarcopenia. Sarcopenia was evaluated from CT images taken within three months preoperatively. Self-reported HRQOL was assessed preoperatively at trial enrollment and eight weeks postoperatively with the HRQOL questionnaire Short Form 36 (SF-36). rollment and eight weeks postoperatively with the HRQOL questionnaire Short Form 36 (SF-36).
Results: There was a significant association between preoperative weight loss and reduced physical HRQOL. No association between sarcopenia and HRQOL was observed. Overall survival was significantly associated with physical HRQOL both pre- and postoperatively, and with postoperative mental HRQOL. The association with survival was particularly strong for postoperative physical HRQOL $(\mathrm{HR}=2.21, \mathrm{p}=0.001)$

Conclusion: There is a strong association between postoperative HRQOL and overall survival after major upper abdominal surgery. 


\section{PO027}

\section{THE IMPLEMENTATION OF ENHANCED RECOVERY AFTER SURGERY (ERAS) PROTOCOL IN GYNAECOLOGY DEPARTMENT - THE FOLLOW-UP AT 1 YEAR}

Agnieszka Biskup ${ }^{1}$, Aleksandra Taszarek ${ }^{1}$, Tomasz Nikodemski ${ }^{*}$, Anita Chudecka-Glaz ${ }^{1}$, Aneta Cymbaluk-Ploska ${ }^{1}$

${ }^{1}$ Pomeranian Medical University, ${ }^{2}$ SP ZOZ MSW, Szczecin, Poland

Objectives: To establish the progress of implementation process of ERAS pathway. We aim for implementation level for each task above $70 \%$.

Methods: Retrospective analysis of two sets of 100 consecutive medical records, patients treated before (PRE) and after (POST) introduction of the ERAS protocol. All patients underwent major gynaecological surgery.

Results: Not every aspect of introduced protocol was fulfilled. Cultural factors heavily hindered the introduction of the ERAS program. Patients in Poland strongly belief that lying in bed is essential to recover quicker. It is very difficult to encourage them to get up and have a meal at the table. In their opinion medical staff is indolent and not welling to help. Very often patients demand pre-med because in their opinion it is crucial to have one. They think that, they deserve it because "they have an insurance". Explanation made by medical team were not always convincing for the patients. Achievements of objectives, which depend to a greater extent on the medical staff, have been better completed. It confirms enthusiasm, dedication and extensive knowledge of our medical team. Patient in POST were discharged earlier and a complication rate was lower.

Conclusion: This research shows why expansion of the ERAS idea is so slow. More consultation with patients is mandatory. Information about ERAS protocol in media would facilitate patients' education among conservative society.

To introduce new, innovative methods of treatment, one has to take into account the cultural and ideological factors especially when the patient involvement is essential.

Table 1 Percentage achieved ERAS objectives

\begin{tabular}{|c|c|c|c|}
\hline $\begin{array}{l}\text { ERAS } \\
\text { protocol } \\
\text { goals }\end{array}$ & Pre & Post & $\begin{array}{l}\text { Evaluation } \\
\text { of progress }\end{array}$ \\
\hline No bowel prep & $3 \%$ & $15 \%$ & poor \\
\hline No fasting & $\begin{array}{l}\text { last meal - noon } \\
\text { day before } \\
\text { surgery }\end{array}$ & $\begin{array}{l}\text { last meal - evening } \\
\text { day before surgery }\end{array}$ & poor \\
\hline No pre-med & $5 \%$ & $30 \%$ & poor \\
\hline $\begin{array}{l}\text { Restrictive iv fluid } \\
\text { regime }\end{array}$ & $2 \%$ & $22 \%$ & poor \\
\hline $\begin{array}{l}\text { Laparoscopic } \\
\text { technic }\end{array}$ & $12 \%$ & $44 \%$ & satisfactory \\
\hline $\begin{array}{l}\text { Routine } \\
\text { mobilisation }\end{array}$ & $\begin{array}{l}\text { day of surgery - } \\
0 \%, \\
1 \text { post op day }-86 \%\end{array}$ & $\begin{array}{l}\text { day of surgery }-45 \% \text {, } \\
1 \text { post op day }-54 \%\end{array}$ & satisfactory \\
\hline $\begin{array}{l}\text { Non-opioid oral } \\
\text { analgesic }\end{array}$ & $20 \%$ & $88 \%$ & good \\
\hline $\begin{array}{l}\text { Prevention of } \\
\text { nausea and } \\
\text { vomiting }\end{array}$ & $56 \%$ & $86 \%$ & good \\
\hline $\begin{array}{l}\text { Early removal of } \\
\text { catheters } \\
\text { (in } 24 \mathrm{~h} \text { ) }\end{array}$ & $7 \%$ & $70 \%$ & good \\
\hline $\begin{array}{l}\text { Perioperative oral } \\
\text { nutrition }\end{array}$ & $\begin{array}{l}\text { day of surgery - } \\
1 \% \text {, } \\
1 \text { post op day }-8 \%\end{array}$ & $\begin{array}{l}\text { day of surgery }-13 \% \\
1 \text { post op day }-79 \%\end{array}$ & good \\
\hline
\end{tabular}

\section{PO028}

\section{IMPLEMENTATION OF AN ERAS PROTOCOL ACROSS A LARGE HEALTHCARE SYSTEM: THE ALBERTA ERAS COLORECTAL SURGERY EXPERIENCE}

Gregg Nelson $^{*}{ }^{1}$, Ellen T. Crumley ${ }^{2}$, Lawrence Kiyang ${ }^{2}$, Leah Gramlich $^{2}$

${ }^{1}$ Tom Baker Cancer Centre, Calgary, ${ }^{2}$ University of Alberta, Edmonton, Canada

Objectives: The implementation of most ERAS protocols has occurred primarily in standalone hospitals and institutions worldwide. In Alberta, Canada there is an initiative to implement system-wide protocols within a provincial governance structure. Our objective was to examine the effect of provincial implementation of the ERAS colorectal protocol in six sites. Methods: ERAS Implementation Programs for colorectal surgery were carried out systematically in six major hospitals (Peter Lougheed Centre and Grey Nuns Hospital 2013; Royal Alexandra Hospital, Misericordia Community Hospital, University of Alberta Hospital, and Foothills Medical Centre 2014). Data was extracted from the ERAS Interactive Audit System (EIAS). The main outcomes included the effect of ERAS implementation on length of stay (LOS), care element compliance, and complication rates.

Results: From September 2013 to December 2014, 1090 patients were evaluated across the six sites (350 patients pre-ERAS implementation, 740 patients post-ERAS implementation). Mean patient age was 62 years in both groups. For the 6 sites, the main outcomes were: reduction in length of stay from a baseline median of 6 days to a median of 4 days at 12 months of implementation, increase in care element compliance of $21.8 \%$

Table 1 Effect of ERAS implementation on length of stay, care element compliance, and complication rates

\begin{tabular}{llllll}
\hline $\begin{array}{l}\text { Time after } \\
\text { guideline } \\
\text { Implementation }\end{array}$ & $\begin{array}{l}\text { Compliance } \\
\text { (mean \%) }\end{array}$ & $\begin{array}{l}\text { LOS } \\
\text { (Median } \\
\text { number } \\
\text { of days) }\end{array}$ & $\begin{array}{l}\text { Wilcoxon } \\
\text { P value }\end{array}$ & $\begin{array}{l}\text { Complications } \\
\text { Rate Ratio } \\
\left(C^{*}\right)\end{array}$ & $\begin{array}{l}\text { Wald } \\
\text { Test } \\
P \\
\text { value }\end{array}$ \\
\hline Baseline & 39.6 & 6 & & & \\
ERAS Patients & 61.4 & 5 & $<.0001$ & $0.93(0.82-1.01)$ & 0.2591 \\
3 months & 63.7 & 5 & 0.0023 & $0.87(0.74-1.04)$ & 0.1209 \\
6 months & 62.8 & 5 & $<.0001$ & $1.00(0.83-1.20)$ & 0.9990 \\
9 months & 58.6 & 5 & 0.0444 & $0.89(0.74-1.09)$ & 0.2733 \\
12 months & 60.3 & 4 & $<.0001$ & $0.98(0.79-1.22)$ & 0.8664 \\
\hline
\end{tabular}

*Confidence Interval

from baseline, and a complications rate ratio of 0.93 for ERAS patients compared to baseline (Table 1).

Conclusion: Systematic implementation of an ERAS colorectal program across a large healthcare system results in benefits similar to those obtained in smaller standalone implementations. Future work includes identifying barriers/enablers to the implementation of ERAS in large healthcare systems and also comparing single-protocol vs. multiple-protocol implementation.

Disclosure of Interest: None Declared.

Disclosure of Interest: None Declared. 


\section{PO029}

\section{LAPAROSCOPY IMPROVES PERIOPERATIVE OUTCOMES AND COST IN ENHANCED RECOVERY COLORECTAL SURGERY}

Mohamed Abdelgadir Adam ${ }^{*}{ }^{1}$, Zhifei Sun ${ }^{1}$, Jina Kim ${ }^{1}$, Julie Thacker ${ }^{1}$

${ }^{1}$ Department of Surgery, Duke University Medical Center, Durham, United States

Objectives: Patients undergoing colorectal surgery in the setting of enhanced recovery protocols (ERP) are well optimized, with the various components of these protocols. In the U.S., laparoscopy is frequently used in the setting of ERPs; however, data are scarce regarding the cost-effectiveness of this technique and whether it provides significant improvement to offset the higher operative cost associated with laparoscopy. Our aim was to examine short-term outcomes and inflation-adjusted costs between laparoscopic vs. open colorectal surgery within an ERP.

Methods: Patients undergoing major, elective colorectal resections under an ERP were included from a prospectively maintained institutional database (2010-2013). Descriptive and multivariable statistics were employed to compare short-term outcomes, length of stay and costs from laparoscopic vs. open colorectal resections.

Results: A total of 675 patients were included; 391 had laparoscopic and 284 open surgery. Age, race, BMI, ASA scores, and diagnosis were similar between the two groups. Compared to open, laparoscopic surgery was associated with lower rates of postoperative ileus $(8 \% \mathrm{vs}$ $19 \%)$ and major complications (11\% vs $26 \%)$; shorter hospital length of stay (mean 5 vs 8 days), and reduced total hospital cost (mean $\$ 10,344$ vs $\$ 12,858$ ) also associated with laparoscopy (all $\mathrm{p}<0.01$ ). Operative cost was higher in the laparoscopic group (mean $\$ 5,912$ vs $\$ 5,834, \mathrm{p}=0.02)$. However, after adjustment for demographics, BMI, ASA scores, diagnosis, alvimopan and epidural use, and extent of resection, patient undergoing laparoscopic vs open surgery had a lower likelihood of having a major complication (OR $0.27,95 \% \mathrm{CI}$ $0.16-0.45, \mathrm{p}<0.0001)$ and had a shorter length of stay by -1 day $(\mathrm{p}<0.0001)$ and reduced total hospital cost by $-\$ 894(\mathrm{p}<0.0001)$. Conclusion: Use of laparoscopy for colorectal resections appears to confer additional improvement to enhanced recovery protocols, with improvement in postoperative morbidity and hospital length of stay. Laparoscopic colorectal surgery within an enhanced recovery protocol is cost-effective, offsetting the higher operative costs of laparoscopy by a significant margin. Laparoscopy may therefore be considered a component of enhanced recovery colorectal surgery protocols.

Disclosure of Interest: None Declared.
PO030

\section{IMPROVING THE APPLICATION OF INTRAOPERATIVE FLUID MANAGEMENT TECHNOLOGY AT UNIVERSITY COLLEGE HOSPITAL, LONDON}

Matthew Higham ${ }^{*}{ }^{1}$, Sanjiv Patel $^{1}$, Kirstie McPherson ${ }^{1}$, Monty Mythen $^{2}$

${ }^{1}$ Anaesthetics, University College Hospital, ${ }^{2}$ Centre for Anaesthesia, University College London, London, United Kingdom

Objectives: Evidence has shown that the use of intraoperative fluid management technology (IOFMT) can result in improved outcomes for patients and benefits for the health economy (1). More specifically, IOFMT used in a protocolised manner improves outcomes in high risk surgical patients (2), contributing to enhanced recovery. The NHS has identified the use of IOFMT in certain procedures as one of six high impact innovations that are requirements for incentive payments (3).

We aimed to audit the use of IOFMT at our institute, both before and after the introduction of local perioperative fluid guidelines, including an IOFMT algorithm.

Methods: We collected data for all surgical patients undergoing procedures in which the use of IOFMT is advised by the NHS (1). We collected the data over a three day period, recording which IOFMT was used and whether an algorithm for its use and interpretation was followed. The same process was repeated following local teaching and the introduction of perioperative fluid guidelines and IOFMT algorithm.

Results: 56 patients were included in the first round of data collection, and 47 in the second. 10 and 7 of these, respectively, underwent procedures for which IOFMT is encouraged with incentive payments by the NHS. Only $20 \%$ of patients from the first round had IOFMT used during their surgery, and none with an algorithm to guide its use. This increased to $43 \%$ in the second round, all of which were guided by the newly introduced algorithm.

Conclusion: We demonstrated an improvement in adherence to recommendations regarding use of IOFMT after an intervention of education alongside new perioperative fluid guidelines. There is clearly further improvement to be made, which will ultimately result in improved patient outcomes and benefits to the local health economy.

Disclosure of Interest: None Declared. 


\section{PO031}

\section{IMPLEMENTATION OF AN ENHANCED RECOVERY PROGRAM AT A TERTIARY CANCER CENTER}

Alpa Nick ${ }^{*}{ }^{1}$, Larissa Meyer ${ }^{1}$, Maria D. Iniesta ${ }^{1}$, Javier LaSala ${ }^{2}$, Melinda Harris ${ }^{1}$, Lindsey Palmer ${ }^{1}$, Terri W. Earles ${ }^{1}$, Katherine

E. Cain ${ }^{3}$, LaKisha Washington ${ }^{4}$, Mark Munsell ${ }^{5}$, Pedro T. Ramirez ${ }^{1}$

${ }^{1}$ Gynecologic Oncology and Reproductive Medicine,

${ }^{2}$ Anesthesiology \& Perioperative Medicine, ${ }^{3}$ Division of Pharmacy, ${ }^{4}$ Division of Nursing, ${ }^{5}$ Biostatistics, UT MD Anderson Cancer Center, Houston, United States

Objectives: To implement a multidisciplinary enhanced recovery program (ERP) for all patients undergoing exploratory laparotomy for gynecologic indications at a tertiary cancer center.

Methods: Interventions included allowing oral intake of fluids up to 2 hours before induction of anesthesia; pre-, intra-, and post-operative euvolemia as well as opioid-sparing analgesia (total intravenous anesthesia); and ambulation on the day of surgery. Consecutive patients managed under an ERP undergoing exploratory laparotomy between 11/4/2014 and 1/15/2015 were compared with historical controls (May to November 2014). Wilcoxon rank-sum and Fisher's exact tests were used for comparisons.

Results: A total of 58 enhanced recovery women in the case group were compared with women in the control group. Thus far, enhanced recovery has resulted in a 1-day reduction in hospital stay (median LOS pre-implementation: 4 days [2-27] vs. post-implementation: 3 days $[1-11], \mathrm{p}=0.001$ ) with stable readmission rates (pre-ERP: $11.7 \%$ vs. post-ERP: $12 \%, \mathrm{p}=1.00$ ). No differences were observed in rates of pre-operative and post-operative complications (GI: preERP $24 \%$ vs. post-ERP $15 \%, p=0.26$; GU: pre-ERP $6 \%$ vs. postERP $13 \%, p=0.22$; Neuro: pre-ERP $0.01 \%$ vs. post-ERP $0.02 \%$, $\mathrm{p}=1.0$; Hematologic: pre-ERP $6 \%$ vs. post-ERP $14 \%, \mathrm{p}=0.13$ ). Among those with advanced or recurrent ovarian cancer (pre-ERP $57 \%$; post-ERP $45 \%$ ), median length of return to intended oncologic therapy (RIOT) was 30 days (range 15-52) pre-ERP compared to 22 days (range 20-41) post-ERP ( $\mathrm{p}=0.08$ )

Conclusion: Implementation of an ERP at a tertiary cancer center is feasible. Early evaluation has already resulted in reduced length of stay with stable readmission and morbidity rates. Further study is warranted to determine impact on RIOT.

Disclosure of Interest: None Declared.

\section{PO032}

\section{ENHANCED RECOVERY PATHWAY FOR HEAD AND NECK CANCER FREE FLAP SURGERY AND RECONSTUCTION; EVALUATING THE USE AND NEED FOR PERIOPERATIVE CENTRAL VENOUS CATHETERS}

Kirstie Mcpherson ${ }^{*}{ }^{1}$, Gautam Kumar ${ }^{1}$, Irene Bouras ${ }^{1}$, Ed Burdett ${ }^{1}$, Monty Mythen ${ }^{2}$

${ }^{1}$ Anaesthetics, University College Hospital, London, ${ }^{2}$ Anaesthetics \& Critical Care, University College London, London, United Kingdom

Objectives: Our institution is one of the largest centres in the United Kingdom offering complex head and neck cancer free flap surgery. Operations are frequently long, complex, requiring microvascular reconstruction and the input of a skilled multidisciplinary team. The service has recently undergone change to incorporate many of the elements of enhanced recovery, and all steps of the patient's pathway have been interrogated to evaluate quality, streamlining and standardising delivery of care. An important element within this evaluation was the need for central venous catheters (CVC's), which until recently was de rigeur for the majority of patients undergoing long and complex surgery.

Methods: We retrospectively reviewed the case notes for all patients undergoing head and neck free flap surgery in our institution over a two year period (1. January 2012-1. January 2014 inclusive). Data collected from notes included presence of a CVC, length of stay in intensive care, time period to removal/exchange, and most importantly, use of CVC. Uses included administration of inotropes, parenteral nutrition, electrolytes and central venous pressure monitoring. All data was anonymysed and securely analysed using excel. This work represented service evaluation and did not require ethics approval.

Results:

\begin{tabular}{|c|c|c|c|c|c|c|c|c|}
\hline \multirow[t]{2}{*}{ Patient } & \multirow{2}{*}{$\begin{array}{l}\text { CVC } \\
\text { inserted } \\
\text { for } \\
\text { surgery }\end{array}$} & \multicolumn{4}{|c|}{ Perioperative CVC use } & \multirow{2}{*}{$\begin{array}{l}\text { CVC } \\
\text { duration } \\
\text { (mean } \\
\text { days) }\end{array}$} & \multirow{2}{*}{$\begin{array}{l}\text { ITU } \\
\text { LOS } \\
\text { (mean } \\
\text { days) }\end{array}$} & \multirow{2}{*}{$\begin{array}{l}\text { New } \\
\text { CVC } \\
\text { or } \\
\text { replaced }\end{array}$} \\
\hline & & $\begin{array}{l}\text { Inotro- } \\
\text { pes }\end{array}$ & TPN & $\mathrm{K}+$ & CVP & & & \\
\hline $\mathrm{n}$ & $35 / 46$ & $4 / 35$ & $0 / 35$ & $6 / 35$ & $3 / 35$ & 3.0 & 3.3 & $0 / 46$ \\
\hline$(\%)$ & $76.1 \%$ & $11.4 \%$ & $0 \%$ & $17.1 \%$ & $8.6 \%$ & $106 / 35$ & $154 / 46$ & $0 \%$ \\
\hline
\end{tabular}

$11 / 46(23.9 \%)$ did not receive a CVC prior to surgery. None of these patients went on to require one in ITU. All but one (34/35) CVCs were femoral.

Conclusion: Very few numbers of patients who received a CVC required its use perioperatively. In spite of this, CVCs remained in situ for a mean of 3 days. With an understanding of the morbidity of CVCs (e.g. infection and metastatic seeding), as well as awareness of the importance of preserving vasculature in the event of flap failure, our institution has adopted a policy in line with enhanced recovery maxims with both economic and patient-centred benefits. Unless specifically indicated, CVCs are not routinely required for head and neck free flap surgery.

Disclosure of Interest: None Declared. 


\section{PO033}

\section{FACTORS INFLUENCING POSTOPERATIVE PAIN IN PATIENTS UNDERGOING AUTOLOGOUS BREAST RECONSTRUCTION IN AN AMBULATORY SETTING}

Kathleen Armstrong ${ }^{*}{ }^{1}$, Kristen Davidge ${ }^{1}$, Mitchell Brown ${ }^{1}$, Pamela Morgan $^{2}$, Mary Li $^{3}$, Lisa Cunningham ${ }^{3}$, John Semple ${ }^{1}$

${ }^{1}$ Division of Plastic \& Reconstructive Surgery, Department of Surgery, ${ }^{2}$ Department of Anaesthesia, University of Toronto, ${ }^{3}$ Department of Anesthesia, Women's College Hospital, Toronto, Canada

Objectives: An increasingly complex and diverse number of procedures are performed in an ambulatory setting. Appropriate postoperative pain management is critical to preventing unnecessary readmissions and improving patient-reported satisfaction and postoperative quality of recovery. ${ }^{1,2}$ This study assesses factors that influence postoperative pain in patients undergoing autologous breast reconstruction in an ambulatory setting.

Methods: This study performs a secondary analysis of data collected in a prospective autologous breast reconstruction cohort at Women's College Hospital. Postoperative pain was reported using a $0-10$ numeric pain rating scale on postoperative day (POD) $1,2,4$ and 7 . Forty patients (160 observations) were included in this study. A random effect panel model was used to evaluate the impact of type of procedure, preoperative pain, body mass index (BMI), and age on postoperative pain.

Results: Results: Pain scores improved over the postoperative period. Patients who underwent a pedicled latissimus dorsi flap breast reconstruction reported higher pain scores than patients who underwent a pedicled transverse rectus abdominis flap breast reconstruction $(\mathrm{p}=0.026)$. Postoperative pain was positively correlated with preoperative pain $(\mathrm{p}=0.001)$. Postoperative pain was

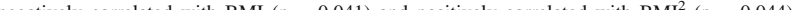
flecting a quadratic relationship in which patients with low and high BMIs report higher postoperative pain scores. Within the multivariate model, there was no statistically significant association between postoperative pain and age.

Conclusion: Several predictive factors of postoperative pain after ambulatory autologous breast reconstruction were identified in this study. These factors should be considered when planning postoperative analgesia.

Disclosure of Interest: None Declared.

\section{PO034}

\section{ENHANCED RECOVERY AFTER THORACIC SURGERY: OUTCOMES FOLLOWING IMPLEMENTATION OF A TAILORED ERAS PATHWAY IN A TERTIARY CENTRE}

Roxanna Zakeri $^{*}{ }^{1}$, Hetal Patel ${ }^{1}$, Cashel Norcliffe ${ }^{1}$, Andrew Middleton ${ }^{1}$, Jagan Rao ${ }^{1}$, John Edwards ${ }^{1}$, Alison Parnell ${ }^{2}$, Laura Socci $^{1}$

${ }^{1}$ Cardiothoracic Surgery, ${ }^{2}$ Anaesthesia, Sheffield Teaching Hospitals NHS Foundation Trust, Sheffield, United Kingdom

Objectives: Minimally-invasive video-assisted thoracoscopic surgery (VATS) and evolution of pulmonary rehabilitation services have enabled ERAS principles to be applied to thoracic surgery, aiming to reduce postoperative recovery time and complications. We present our tailored, multidisciplinary ERAS protocol and results for patients undergoing lung resection in a large, tertiarylevel centre.

Methods: Retrospective case-note analysis of 108 consecutive anatomical lung resections performed before and after implementation of our ERAS protocol (54 patients in each cohort) during the periods January-April 2014 and July-October 2014. A total of 32 key variables were analysed in 3 groups: preoperative optimisation (e.g. smoking cessation, carbohydrate loading, benzodiazepine avoidance), intraoperative factors (minimally-invasive technique, regional anaesthesia), postoperative outcomes (analgesia, complications, readmission).

Results: No significant differences in median age, surgical indication or staging were found between the cohorts. Compliance with ERAS preoperative measures was strong: in particular, use of benzodiazepine sedation fell significantly $(35.2 \%$ vs $5.6 \%, \mathrm{p}<0.001)$. More patients were admitted on the day of surgery $(3.7 \%$ vs $68.5 \%, \mathrm{p}<0.001)$. Minimally-invasive surgery increased by two-thirds to $51.9 \%$ and we have moved completely from epidural to regional anaesthesia with paravertebral and intercostal blocks $(13.0 \%$ vs $87.0 \%, \mathrm{p}<0.001)$. Postoperative cardiovascular complications markedly reduced (combined AF/MI/DVT/PE) $(24.1 \%$ vs $7.5 \%, \mathrm{p}=0.019)$, as did constipation rate $(33.3 \%$ vs $13 \%, \mathrm{p}=0.012)$. Decreases were also seen in chest and wound infection rates. Readmission to level-3 care halved (7.4\% vs 3.7\%). Median length of stay (LOS) with ERAS was 5 days, a reduction by 1 day. Notably, median LOS following pneumonectomy fell from 8 to 5.5 days. Conclusion: Our results demonstrate that ERAS can be applied to thoracic surgery patients with great success. Most common and major postoperative complications have been reduced with a resulting decrease in LOS.

Disclosure of Interest: None Declared.

\section{PO035}

\section{POSTOPERATIVE QUALITY OF RECOVERY SCALE - AN EFFICIENT AND OBJECTIVE TOOL FOR PERIOPERATIVE CARE ASSESSMENT IN CARDIAC SURGERY}

David Gelman $^{*}{ }^{1}$, Edmundas Sirvinskas ${ }^{1}$, Tadas Lenkutis ${ }^{1}$, Greta Kasputyte $^{2}$, Ieva Zujute ${ }^{2}$, Kestutis Petniunas ${ }^{2}$, Aurelija Lukoseviciute $^{2}$, Rimantas Benetis ${ }^{1}$

${ }^{1}$ Department of Cardiac, Thoracic and Vascular Surgery, ${ }^{2}$ Lithuanian University of Health Sciences, Kaunas, Lithuania

Objectives: We aimed to evaluate the feasibility of Postoperative Quality of Recovery Scale (PQRS) in on-pump cardiac surgery and to determine relevant points in the perioperative timeline for early, late and long-term recovery assessment.

Methods: An observational pilot study included 32 consequent patients undergoing elective on-pump cardiac surgery in one center. Original PQRS questionnaire was back translated into Lithuanian language and approved by local ethics committee. All researchers conducted the interviews according to the guidance available online. Given the nature of on-pump cardiac surgery (i.e. usual need of prolonged mechanical ventilation postoperatively) assessment timepoints were chosen as follows: baseline - on a day before surgery, immediate - not assessed, early - 24 hours after surgery with most patients extubated but all them still in ICU, late -7 days after surgery with most patients out of ICU but still in hospital and long term -1 month after surgery with most patients have completed ambulatory rehabilitation program. While in hospital patients had face-to-face interviews and for the long term timepoint they were telephoned. Recovery was defined as return to baseline values or better.

Results: 30 patients completed the study -1 refused to continue, 1 was lost due to incorrect contacts. None of the patients recovered by 24 hours (physiological recovery was $52 \%$, cognitive $-45 \%$ ). A week after surgery complete recovery was achieved in $30 \%$ of the patients - lowest recovery rate was observed in nociceptive (59\%) and cognitive (76\%) domains. By one month only $55 \%$ of the patient were fully recovered - nociceptive $(69 \%)$ and cognitive $(76 \%)$ domains remained the low scorers. Conclusion: With low patient refusal rate, easy to conduct and not time consuming questionnaire PQRS is a very efficient tool for recovery after surgery assessment. Our pilot study showed the (to achieve (a) extensive rehabilitation period cardiac surgery: 1-14 days before and 24 hours, 7 days and 1-3 months after the surgery.

Disclosure of Interest: None Declared.

\section{PO036}

\section{THE EVOLUTION AND IMPACT OF NATIONAL ERAS SOCIETIES: LESSONS TO LEARN FROM ERAS-UK}

Nader Francis ${ }^{*}{ }^{1}$, Mike Scott ${ }^{2}$, Tom Wainwright ${ }^{3}$, David McDonald ${ }^{4}$, Rachael Barlow ${ }^{5}$, Ken Fearon ${ }^{6}$, Olle Ljunggvist ${ }^{7}$, Fiona Carter $^{1}$

${ }^{1}$ Surgery, Yeovil Hospital NHS Foundation Trust, Yeovil, ${ }^{2}$ Anaethesia, Royal Surrey County Hospital NHS Foundation Trust, Guildford, ${ }^{3}$ Orthopaedics, The Royal Bournemouth Hospital, Bournemouth, ${ }^{4}$ Orthopaedics, Scottish Government, Glasgow, ${ }^{5}$ Clinical Academic Fellow, Cardiff University, Cardiff, ${ }^{6}$ Surgery, Edinburgh University, Edinburgh, United Kingdom, ${ }^{7}$ Department of Molecular Medicine and Surgery, University Örebro, Stockholm, Sweden

Objectives: With the growing adoption of Enhanced Recovery After Surgery (ERAS) principles in many countries, there is emerging interest in developing national networks or chapters for ERAS The aim of this study was to evaluate the development and sustainability of a national organisation (ERAS-UK) since it was established in 2010.

Methods: ERAS-UK was set-up as a non-profit network following the first international ERAS conference in Bristol (2010), as an outcome of a consensus process involving the UK stakeholders. This group agreed that setting up an organisation to share information and best practice would be very beneficial. Members have been asked to evaluate the society's activities from 2010-2014. Assessmen of the financial sustainability and the educational impact of this network have also been evaluated. Results: There are more than 480 multidisciplinary members representing several specialities (with an average of $70 \%$ annual increase in membership). ERAS UK attracts a diverse array of professionals across all specialities and the society's conferences are the only meetings that involve all disciplines on perioperative care in the UK. Five annual conferences have been conducted across the UK since 2010 with a total attendance of 1150 delegates representing 12 specialities and 15 disciplines. 110 abstracts have been presented with 30 prizes awarded. The members have had opportunities to vote on preferred hosts, venue and on the content for these events. More than $90 \%$ o participants either agreed or strongly agreed that each event would influence their future practice an more than $50 \%$ of delegates return to this event each year. ERAS-UK has worked successfully with 22 different healthcare companies to secure sponsorship and ensure that fees for delegates are as low as possible. The impact of networking on behavioural change in member's institutions will be presented at the conference.

Conclusion: There is a need for national ERAS societies to address local needs, tailored to national healthcare systems. These societies can promote networking across multiple disciplines and specialities and can be financially sustainable after 5 years. Structuring the framework of the relationships between national networks and the international ERAS society is required. 


\section{PO037}

\section{ESTIMATION OF THE RISK OF RESIDUAL RELAXATION AFTER NMB APLICATION IN LAPAROSCOPIC SURGERY}

\author{
Edward Nicolayenko $^{*}{ }^{1}$, Dimitry Kurenkov ${ }^{1}$, Svetlana Chizevskaya ${ }^{1}$
}

${ }^{1}$ Center of Anaesthesiology and Intensive Care Medicine, Research Clinical Center RRW, Moscow, Russian Federation

Objectives: to assess with objective quantitative NMT monitoring the residual NMB at the moment of tracheal extubation in patients undergoing laparoscopic surgery.

Methods: 30 patients, 53,5 $\pm 8,1$ years old, ASA I-III, underwent laparoscopic surgery (cholecystectomy, appendectomy) with routine NMB management. TOF monitoring of NMT during anesthesia has been hidden from attending anesthesiologists, who performed NMB administration and tracheal extubation in accordance with their daily practice. We assessed the degree of NMT recovery at routine extubation, and its time delay after end of surgery.

Results: in 21 patients the reversal of neuromuscular blockade has not been applied. In this group in 15 patients tracheal extubation has been performed in $10.55 \pm 3,39 \mathrm{~min}$ after the last stitch at the TOF $43-81 \%(65 \pm 18 \%)$ and only in 6 pts at the TOF $>90 \%$. Attending anesthesiologists applied sugammadex $(2 \mathrm{mg} / \mathrm{kg})$ for reversal in 9 patients, while the level of NMB was $41 \pm 6,5 \% \mathrm{TOF}$. Tracheal extubation in that cases has been performed in less than 4 minutes after the administration of sugammadex $(3,18 \pm 0,73)$ at the TOF $91 \pm 0,83 \%$.

Conclusion: subjective assessment of NMT recovery and making a decision to extubate based on clinical signs cannot completely eliminate the risks of residual paralysis and related adverse events. In the current practice even with short-acting aminosteroid NMB the objective monitoring of NMT and reversal medication (sugammadex) should be considered justified. Objective monitoring of NMT value required to determine the time of intubation, administration of maintenance doses of relaxants, for assessment of reversal efficacy and extubation possibility.

Disclosure of Interest: None Declared.

\section{PO038}

\section{EFFECTS OF PREOPERATIVE CARBOHYDRATES DRINKS ON POSTOPERATIVE OUTCOME AFTER LAPAROSCOPIC CHOLECYSTECTOMY}

Riza Haldun Gundogdu* ${ }^{1}$, Pamir E. Ersoy ${ }^{1}$, Soner Akbaba ${ }^{1}$, Omer Yazicioglu $^{1}$, Ogtay Nasrullayev ${ }^{1}$, Burak Ciftci ${ }^{1}$

${ }^{1}$ General Surgery, Ataturk Training and Research Hospital, Ankara, Turkey

Objectives: Postoperative nausea and vomiting is the most common cause for postoperative period of patients undergoing laparoscopic cholecystectomy. Overnight fasting changes patient metabolic state and influences their perioperative stress response. Preoperative carbohydrate loading may have accelerated recovery and better overall outcome after abdominal surgery. The aim of the study was to investigate the effects of preoperative carbohydrate-rich drinks on postoperative nausea and vomiting and pain after laparoscopic cholecystectomy.

Methods: A total of 100 patients posted for laparoscopic cholecystectomy were in cluded in the study and were randomized into two groups. Group A (experiment)receiving the carbohydrate-rich drink before surgery, group B (controls)-fasting from midnight before surgery. Postoperative nausea and vomiting and visual analogue score for pain were noted and analyzed for $24 \mathrm{~h}$.

Results: Mean score of nausea in 0-6 h in group A was significantly lower as compared to group B $(\mathrm{p}=0.001)$. Difference in mean score of nausea in $6-24 \mathrm{~h}$ between groups was not significant $(\mathrm{p}>0.05)$. Mean score of vomiting in $0-6$ and $6-24 \mathrm{~h}$ in group A was significantly less than group $B(p=0.001)$. Mean score of pain in group A was significantly less than group $B$ in $0-6 \mathrm{~h}(\mathrm{p}=0.001)$ and $6-24 \mathrm{~h}(\mathrm{p}=0.05)$.

Conclusion: Preoperative carbohydrate-rich drink has a beneficial effect in reducing the incidence of postoperative nausea, vomiting and pain in patients undergoing laparoscopic cholecystectomy. Consumption of oral carbohydrate drinks up to $2 \mathrm{~h}$ prior to surgery is not associated with additional complications.

Disclosure of Interest: None Declared.
PO039

\section{AN EVALUATION OF AN ENHANCED RECOVERY PROGRAM ESTABLISHED FOR PATIENTS UNDERGOING OPEN GYNECOLOGIC SURGERY}

Terri W. Earles ${ }^{*}{ }^{1}$, Melinda G. Harris ${ }^{1}$, Lindsey D. Palmer ${ }^{1}$, Larissa A. Meyer ${ }^{1}$, Maria D. Iniesta ${ }^{1}$, Javier D. Lasala ${ }^{2}$, Katherine E. Cain ${ }^{3}$, LaKisha D. Washington ${ }^{4}$, Pedro T. Ramirez ${ }^{1}$, Alpa M. Nick ${ }^{1}$

${ }^{1}$ Department of Gynecologic Oncology, ${ }^{2}$ Department of Anesthesia, ${ }^{3}$ Department of Pharmacy, ${ }^{4}$ Department of Nursing, The University of Texas MD Anderson Cancer Center, Houston, United States

Objectives: To evaluate the metrics implemented in the enhanced recovery program (ERP) after gynecologic surgery. To determine overall patient satisfaction with the ERP. Methods: Patients undergoing an exploratory laparotomy for gynecologic surgery were enrolled in an ERP. Following discharge from the hospital, a 10 question survey was conducted via telephone evaluating overall satisfaction ( 1 being least satisfied and 10 being highly satisfied), preoperative education, activity compliance, pain control (measured on a $0-10$ pain scale with ' 0 ' being no pain and ' 10 ' being severe pain), adherence to an established pain medication regimen, and need for oxycodone refill. With the exception of overall satisfaction and pain control, all questions were answered in a 'yes' versus 'no' format.

Results: A total of 58 enhanced recovery patients were called upon discharge from the hospital. $42(72.4 \%)$ patients participated in the telephone survey. The median scores were 10 (range $=2-10)$ and $5($ range $=0-10)$ for patient satisfaction and pain control, respectively $31 / 42(73.8 \%)$ patients reported receiving preoperative education; 35/42 (83.3\%) patients reported compliance with the activity requirements; $24 / 42(57.14 \%)$ patients reported adherence to the established pain medication regimen; and 10/42 (23.8\%) patients requested refills on oxycodone. Of note, the patients who answered 'no' when questioned about their adherence to the prescribed pain medication regimen reported having little to no pain. Conclusion: Overall patients were highly satisfied with our ERP. Strategies are needed to improve preoperative and discharge education materials.

Disclosure of Interest: None Declared.

\section{PO040}

\section{DEVELOPMENT OF A TOOL TO OPTIMIZE IMPLEMENTATION OF ENHANCED RECOVERY PATHWAYS}

Daniel Galante ${ }^{1}$, Deborah Hobson ${ }^{1}$, Anne Chin ${ }^{1}$, Hannah Bogen ${ }^{1}$, Andrew Benson ${ }^{2}$, Christopher Wu ${ }^{2}$, Sandy Fang ${ }^{1}$, Bashar Safar ${ }^{1}$, Susan Gearhart ${ }^{1}$, Jonathan Efron ${ }^{1}$, Peter Pronovost ${ }^{3}$, Elizabeth Wick ${ }^{1}$

${ }^{1}$ Department of Surgery, ${ }^{2}$ Department of Anesthesia and Critical Care Medicine, ${ }^{3}$ Armstrong Institute for Patient Safety and Quality, Johns Hopkins University, Baltimore, United States

Objectives: Enhanced Recovery Pathways (ERP) are emerging as one of the most effective strategies to improve the quality and value of surgical care. Successful implementation requires a coordinated team of providers including surgeons, anesthesia providers and nurses. Learning from defects is an important strategy for quality improvement. We hypothesized that detailed evaluation of patients with longer than expected length of stay (LOS) using a standardized outlier investigation tool would identify areas to optimize implementation.

Methods: We developed an ERP with best practices for the prevention of surgical site infection, venous thromboembolism and urinary tract infection and promotion of patien engagement, and then developed a tool to assess compliance. All patients undergoing elective colorectal surgery at Johns Hopkins Hospital from February 1 through September 16, 2014 with a LOS stay greater than 5 days were reviewed. Non-compliance with ERP process measures were surfaced and addressed by a multidisciplinary group of frontline team members. This data was compared to those patients with a LOS of less than 5 days. Results: The ERP resulted in a 2-day reduction in LOS as compared to the prior year. 191 patients $(72.6 \%$ ) had LOS less than 5 days. The remaining 72 patients with a LOS greater than 5 days had an average LOS of 11.1 days. Patients were grouped into one of six categories, based on a generalized reason for prolonged length of stay. The categories were: ileus (58\%), sepsis $(5.5 \%)$, post-operative complications $(5.5 \%)$, technical complications $(10 \%)$, comorbidities $(8 \%)$, and other $(13 \%)$. There were significant differences in pathway compliance in patients with a LOS greater than 5 days as compared to less than 5 days. Analysis of patients with a LOS greater than 5 days due to ileus revealed increased IV fluid intake and decreased post-operative mobility as compared to patients with a LOS less than 5 days.

Conclusion: An ERP is challenging to implement; despite a multidisciplinary team approach, many patients did not receive all of the bundled processes. Patients with a longer LOS were more likely to be non-compliant with key ERP processes. Standardized evaluation of outlier patients allowed the team to identify areas to target for enhanced compliance. 


\section{PO041}

\section{SPANISH SURVEY ON UNINTENDED PERIOPERATIVE HYPOTHERMIA}

Alberto Gutiérrez Martínez ${ }^{1}$, Antonio Domínguez Chaos ${ }^{1}$, Javier Ripollés Melchor ${ }^{1}$, Eugenio Martínez Hurtado ${ }^{1}$, Verónica Guilló Moreno $^{2}$, Rubén Casans Francés ${ }^{3}$, Alfredo Abad Gurumeta ${ }^{4}$, José Manuel Ramírez ${ }^{*}$, José María Calvo Vecino ${ }^{1}$ on behalf of GERM. Grupo Español de Rehabilitación Multimodal

${ }^{1}$ Anesthesia and Critical Care, Hospital Universitario Infanta Leonor, ${ }^{2}$ Anesthesia and Critical Care, Hospital Universitario Puerta de Hierro, Madrid, ${ }^{3}$ Anesthesia and Critical Care, Hospital Universitario Lozano Blesa, Zaragoza, ${ }^{4}$ Anesthesia and Critical Care, Hospital Universitario La Paz, Madrid, ${ }^{5}$ General Surgery, Hospital

Universitario Lozano Blesa, Zaragoza, Spain

Objectives: Hypothermia, defined as a core body temperature less than $36^{\circ} \mathrm{C}\left(96.8^{\circ} \mathrm{F}\right)$, is a relatively common occurrence in the unwarmed surgical patient. A mild degree of perioperative hypothermia can be associated with significant morbidity and mortality. Preventing intraoperative hypothermia is recommended with a high level of evidence by ERAS guidelines. Attempts should be made to avoid hyothermia because it increases the risk of perioperative complications. The aim of our study is to assess the degree of knowledge of Unintended Perioperative Hypothermia (UPH) in Spanish anaesthesiologists.

Methods: Between March and April 2013, we collected data from postoperative internet surveys of anaesthesiologists in our country. This survey was answered with their own knowledge and included data of prevention, monitoring, preoperative, intraoperative and postoperative measures and interventions. We excluded patients in whom the data collection form was not complete. Data were analyzed with Excel 2011 and SPSS 20.0. Tables and histograms were also completed with SPSS 20.0.

Results: 328 anaesthesiologists answered the survey. $48 \%$ of anaesthesiologists consider hypothermia in patients undergoing surgery, $42 \%$ usually monitor temperature in patients during surgery, $47 \%$ consider monitoring temperature important, $47 \%$ assess any type of prevention. Data about opinions were also collected.

Conclusion: Monitoring of body temperature and avoidance of unintended perioperative hypothermia through active and passive warming measures are the keys to preventing its complications. The survey showed the large unknowledge about the issue. We are continuing our study in order to determine specific interventions to awareness anaesthesiologists about it.

Disclosure of Interest: None Declared.

\section{PO042}

\section{ERAS IN COLORECTAL SURGERY, EFFECTIVENESS OF} REGIONAL ANALGESIA. OUR EXPERIENCE

Carolina Yepes ${ }^{*}{ }^{1}$, Luisa Martinez ${ }^{1}$, Gracia Cardenas ${ }^{1}$, Laura Martinez ${ }^{1}$, Pera Poch $^{1}$

${ }^{1}$ Anestesia, Hospital General Granollers, Barcelona, Spain

Objectives: Evaluate the effectiveness of two analgesic techniques in patients undergoing colorectal surgery within ERAS protocol.

Methods: Comparative retrospective study, by review of medical records. Population: patients undergoing colorectal surgery in our center during 2013. They are divided according received thoracic epidural analgesia (GP) or TAP block (GT). Variables studied: demographics, procedure, morphine requirements and EVA value during the first 6 postoperative hours, start sitting and intake by mouth, length of stay (LOS) and rates of readmission and reoperation. Results: We collect 40 cases in GT and 51 in GP. Mean age of 69 years $( \pm 12.2), 63 \%$ male and $96 \%$ were ASA $2-3$. Both groups were homogeneous for age, sex and BMI. Laparoscopy $37 \%$ in GT and $29 \%$ in GP. Morphine consumption during the first 6 hours after surgery was $1.7( \pm 3.1)$ and $6.79( \pm 5)$ mgr for GP and GT respectively. No statistically significant differences in relation to EVA in the immediate postoperative, LOS or in rates of hospital readmission and reoperation.

Conclusion: Both analgesic techniques have similar effectiveness. The LOS was not affected by the analgesic technique when ERAS program was used.

Disclosure of Interest: None Declared. 


\section{PO043}

\section{A 5-YEAR LENGTH OF STAY TREND ANALYSIS FOR SURGICAL PROCEDURES IDENTIFIED BY THE DEPARTMENT OF HEALTH AS SUITABLE FOR ERAS IN THE SOUTH WEST REGION OF ENGLAND}

Thomas W. Wainwright ${ }^{*} 1$

${ }^{1}$ Orthopaedic Research Institute, Bournemouth University, Bournemouth, United Kingdom

Objectives: In 2009/10 the Department of Health (DOH) launched the Enhanced Recovery Partnership Programme (ERPP). The programme identified colorectal, urology, gynaecology, and musculoskeletal surgical procedures. This paper presents the effect or ERAS introduction on aggregated mean length of stay (LOS) for the DOH identified procedures across 17 hospitals in the South West region of England.

Methods: Dr Foster software was used to interrogate Hospital Episode Statistic data. Elective spells from Jan 2009 until Oct 2014 were analysed, and aggregated mean LOS was calculated for each calendar year. The procedures chosen for analysis were those identified by the DOH ERPP, and included colorectal procedures, cystectomy, prostatectomy, hysterectomy, hip replacement, and knee replacement. OPCS codes and HRG codes detailed in DOH ERPP literature were used to construct the data queries.

Results: Over the 5-year time period from Jan 2009 until Oct 2014, across the region aggregated mean LOS reduced for all surgical procedures. In 2014, case-mix adjusted expected LOS figures show that the mean LOS for the colorectal procedures, cystectomy, hysterectomy, prostatectomy, and total hip replacement was between 0.2 and 1.4 days less than expected. Only total knee replacement had a longer LOS than expected for case-mix (0.3 days).

\begin{tabular}{lrrrrrc}
\hline Procedure & \multicolumn{6}{c}{ Mean Length of Stay (LOS) in days } \\
\cline { 2 - 7 } & 2009 & 2010 & 2011 & 2012 & 2013 & 2014 \\
\hline Colorectal & 9.9 & 8.9 & 8.1 & 7.9 & 8.1 & 7.7 \\
Cystectomy & 16.2 & 15.8 & 14.4 & 14.1 & 13.6 & 14 \\
Hysterectomy & 3.6 & 3.2 & 2.8 & 2.6 & 2.6 & 2.4 \\
Prostatectomy & 3.2 & 2.9 & 2.8 & 2.6 & 2.7 & 2.6 \\
Total Hip replacement & \multicolumn{1}{c}{5} & 6.4 & 6 & 5.7 & 5.6 & 5.5 \\
Total Knee replacement & 5.5 & 5.2 & 5.1 & 4.9 & 5.1 & 4.6 \\
\hline
\end{tabular}

Conclusion: The introduction of ERAS within the UK has helped to reduce mean LOS for a range of surgical procedures over the last 5 years in the South West region. Given that mean LOS was lower than the national case-mix adjusted LOS in the last year for 5 of the 6 procedures, it indicates that there is significant scope for further reduction in LOS across the region and country when the results of international exemplar ERAS sites are compared. The data may suggest that ERAS adoption has not been as complete as previously thought and reported, both within individual hospitals and across hospitals.

Disclosure of Interest: None Declared.

\section{PO044}

13 YEARS OF OPEN AORTIC SURGERY AND LARYNGEAL MASK: A CONVINCING SUPPORT TO VASCULAR ERAS PATHWAY?

Luca Gramaglia $^{*}{ }^{1}$, Alessandra Renghi ${ }^{1}$, Francesco Della Corte ${ }^{1}$

${ }^{1}$ Anaesthesia and Intensive Care I, Novara University Hospital, Novara, Italy

Objectives: Since ProSeal laryngeal mask arway (PSLMA) ${ }^{1}$ has been presented, our anesthesiologists have been evaluated its use in open abdominal aortic surgery, aiming to reduce perioperative stress, preserving airway safety and fast-track pathway ${ }^{2}$.

Methods: A series of open abdominal aortic surgery was recorded to examine effectiveness, difficulties and side effects of PSLMA. Emergent/urgent aortic diseases and endovascular procedures were excluded. After premedication, thoracic epidural catheter was performed if possible. Then correct position of PSLMA was supported by capnography and tidal volume. Spontaneous breathing or assisted ventilation was preserved and curare drug administered only on surgical demand shifting to controlled ventilation as long as required. Recurring hemogasanalysis were performed. At the end of surgery patient was awakened and monitored in recovery room, therefore transferred to surgical ward.

Results: 800 of 1014 patients scheduled were ASA III and IV; PSLMA was used in 943; endotracheal tube in 70; 1 case of tracheostomy cannula. Median $\mathrm{SpO}_{2}(\%)$ : preoperative 97,5; end of surgery 97,3 . Median $\mathrm{ETCO}_{2}\left(\mathrm{cmH}_{2} \mathrm{O}\right)$ : beginning of surgery 35,42 ; end of surgery 36,87 . Intermittent positive pressure ventilation for all time: $12 \%$; only during clamping phase: $32 \%$; spontaneous breathing/ assisted ventilation for all time: $55 \%$. Median $\mathrm{pH}$ preoperative: 7,38 ; end of surgery: 7,33. Median $\mathrm{PCO}_{2}(\mathrm{mmHg})$ preoperative: 43 ; end of surgery: 49. Median $\mathrm{PO}_{2}(\mathrm{mmHg})$ preoperative: 92 ; end of surgery: 95. No one episode of laringospasm after PSLMA removal. Pulmonary complications (patients): 3. Death (patients): 17.

Conclusion: If an anaesthesiologist is already experienced LMA, the PSLMA is easier to use. From our own experience, this device allows anaesthesia management as well as with endotracheal tube. Nevertheless avoiding perioperative stress, reducing amount of muscle relaxant drugs, maintaining airway safety with effective ventilation and fast awakening could contribute to an ERAS programme for major vascular surgery.

Disclosure of Interest: None Declared. 


\section{PO045}

\section{LAPAROSCOPIC AND OPEN RIGHT-SIDED COLONIC RESECTION IN DAILY ROUTINE PRACTICE. A PROSPECTIVE MULTICENTRE STUDY WITHIN AN ERAS PROTOCOL}

Daniel Asklid ${ }^{*}{ }^{1}$, Marit Tiefenthal ${ }^{2}$, Fredrik Hjern ${ }^{1}$, Peter Matthiessen $^{3}$, Ulf Gustafsson ${ }^{1}$

${ }^{1}$ Dep of Surgery, Danderyd University Hospital Department, Department of Clinical Sciences, Danderyd Hospital, Karolinska Institutet, Stockholm, Sweden, ${ }^{2}$ Department of Surgery, Ersta Hospital, Department of Molecular Medicine and Surgery, Karolinska Institutet, Stockholm, Sweden, Stockholm, ${ }^{3}$ Dept of Surgery, Örebro University Hospital, Örebro University, Örebro, Sweden, Örebro, Sweden

Objectives: This study investigates outcomes in laparoscopic compared to open surgery in daily practice when performed in a strict Enhanced Recovery after surgery (ERAS) environment (1).

Methods: In three Swedish ERAS-centres, 292 consecutive patients operated electively for cancer or adenoma in the right colon, in the period $1^{\text {st }}$ of January 2011 until $31^{\text {th }}$ of December 2012, were prospectively registered in a web-based ERAS-database. Peri-operative data were collected from the database and patient charts. Primary outcomes were postoperative recovery and morbidity. Secondary outcome was preoperative selection of patients in relation to the choice of laparoscopic or open technique.

Results: 123 (42\%) patients were selected for laparoscopic surgery. The overall preoperative ERAS-compliance rate was $87 \%$ and no significant difference was seen between surgical techniques. In multivariate analysis, patients operated with laparoscopy had significantly earlier pain control $2.4 \pm 3.2$ days vs. $4.2 \pm 5.9(\mathrm{p}=0.016)$ and shorter length of hospital stay (LOS) 4 vs. 6 days ( $p=0.002)$ compared to open surgery. There was no significant difference in complication rate $18.7 \%$ vs. $21.3 \%$, OR $1.0(0.5-2.0)$, number of removed lymph nodes or rate of R0-resection between laparoscopic and open surgery respectively. Tumours selected for laparoscopy were generally smaller, had a lower T-stage and were predominantly situated in the caecum and the ascending colon compared to patients selected for open surgery.

Conclusion: The use of laparoscopy in routine right-sided colectomy in an ERAS environment, with data on outcome corrected for selection bias, results in faster recovery compared to open surgery.

Disclosure of Interest: None Declared.

\section{PO046}

\section{UTILITY OF ACTIVITY LOGS IN AN ENHANCED RECOVERY PATHWAY IN GYNECOLOGIC SURGERY}

Maria D. Iniesta ${ }^{*} 1$, Maria G. Patrono ${ }^{2}$, Alpa M. Nick ${ }^{1}$, LaKisha D. Washington ${ }^{3}$, Kenia E. Ramos ${ }^{3}$, Terri W. Earles ${ }^{1}$, Javier D. Lasala ${ }^{4}$, Pedro T. Ramirez ${ }^{1}$, Larissa A. Meyer ${ }^{1}$

${ }^{1}$ Department of Gynecologic Oncology and Reproductive Medicine, MD Anderson Cancer Center, Houston, United States, ${ }^{2}$ Departamento de Ginecologia, Hospital Italiano de Buenos Aires, Buenos Aires, Argentina, ${ }^{3}$ Department of Nursing, ${ }^{4}$ Department of Anesthesiology and Perioperative Medicine, MD Anderson Cancer Center, Houston, United States

Objectives: To evaluate the compliance and efficacy of the use of activity logs in patients undergoing exploratory laparotomy for gynecologic indications being treated under the enhanced recovery program (ERP) in our center. Many ERP encourage patients to eat all meals in chair, minimize bed rest, and ambulate frequently in the immediate postoperative setting. It is not clear what "best practices" are to assess compliance with early mobilization goals.

Methods: A non-randomized, quality improvement initiative is ongoing at our center. Since November 2014, all consecutive patients undergoing open gynecologic surgery are treated under the enhanced recovery pathway. Our protocol for early mobilization prescribes being out of bed (OOB) for at least 2 hours and walking at least once on the day of surgery, and increasing this to being OOB for 8 hours and ambulating at least 8 times starting on postoperative day one. In order to assess patient compliance with their physical activity goals, activity $\log$ s were given to the patients upon arrival to the floor by nursing staff. Every morning patients were asked to complete the log and self-report the amount of time spent OOB (sitting included) and walking.

Results: A total of $28(49.1 \%)$ out of 57 patients returned their activity logs. Only one patient completed her activity log every day. Activity on day of surgery was reported by half of the patients. Of these, only $6 / 14(42.9 \%)$ patients met the activity goals for day of surgery. The highest compliance was on first day after surgery, when $67.9 \%$ of the patients completed their activity logs. Over a third of those patients $(35.7 \%)$ self-reported ambulating 8 times and staying OOB for 8 hours.

Conclusion: At this time, activity logs are not a useful method to assess patient activity after surgery. Further ongoing engagement of hospital staff and patients to complete the activity logs may increase compliance. Alternative strategies to track patient activity that do not rely on patients self-report may increase our ability to monitor compliance with physical activity.

Disclosure of Interest: None Declared. 


\section{PO047}

\section{ERAS PROTOCOLS FOR COLORECTAL SURGERY SHOULD BE SUSPENDED AFTER SIGNIFICANT COMPLICATIONS}

Mark Hartney ${ }^{1}$, Sonya Pease ${ }^{*}{ }^{2}$, Yazhou Liu ${ }^{3}$, Rachel A. Karlnoski ${ }^{2}$, Vic Velanovich ${ }^{1}$, Devanand Mangar ${ }^{2}$, Enrico M. Camporesi ${ }^{2}$

${ }^{1}$ Surgery, University of South Florida, ${ }^{2}$ Anesthesiology, Team Health Anesthesia Institute, ${ }^{3}$ Industrial \& Management Sys Engineering, University of South Florida, Tampa, United States

Objectives: To study the link between complications and 30-day readmissions following colorectal surgery using conditional inference trees (CTREES). ${ }^{1}$

Methods: Study exemption was granted by the University of South Florida Institutional Review Board to conduct this analysis. Using deep surgical site infection and return to operating room as potential risk factors and a limited, random subsampling of paired readmissions to non-readmissions, we used CTREE predictive modeling to analyze 2012 ACS-NSQIP colorectal surgical readmission data. Twentyseven variables, including age, ASA classification, total operation time, return to operating room, length of stay, and deep surgical site infection, were analyzed as potential risk factors for readmission.

Results: We reviewed 36445 records. Of these, 3778 were readmitted after colorectal surgery resulting in a readmission rate of $10.4 \%$. The 3778 readmissions were randomly paired with 3778 non-readmissions for the program analysis. CTREES predicted a 98\% risk of readmission within 30 days in patients with deep infections that were discharged $\leq 10$ days after surgery (Table 1 ). Furthermore, a $94 \%$ risk of readmission was predicted for patients who returned to the operating room and were discharged $\leq 9$ days after surgery (Table 1 ).

Table 1 Important variables for 30-day readmission of colorectal surgery patients as determined by CTREES; a subsample of 3778 randomly matched records

\begin{tabular}{lll}
\hline & $\begin{array}{l}\text { Postoperative } \\
\text { duration of } \\
\text { stay }\end{array}$ & $\begin{array}{l}\text { Probability of } \\
\text { Readmission } \\
\text { within 30 days }\end{array}$ \\
\hline Return to & $\leq 9$ days & $94 \%$ \\
Operating Room & $>9$ days & $55 \%$ \\
Organ/Space Surgical & $\leq 10$ days & $98 \%$ \\
Site Infection & $>10$ days & $60 \%$ \\
\hline
\end{tabular}

Conclusion: This retrospective review of colorectal surgery utilizes the CTREES modeling to accurately predict 30 -day readmission rates after development of a deep surgical site infection or after returning to the operating room. Colorectal fast-track protocols are designed to reduce the length of stay following surgery but may fall short in the presence of these 2 complicating factors. A prospective study will be required to actively predict complication risks.

Disclosure of Interest: None Declared.

\section{PO048}

\section{CHOICE OF INTRAVENOUS FLUIDS IN THE EMERGENCY DEPARTMENT}

Kirstie Mcpherson ${ }^{*}{ }^{1}$, Sanjiv Patel ${ }^{1}$, Robert Schulman ${ }^{2}$, Helen Frinta ${ }^{2}$, Monty Mythen ${ }^{3}$

${ }^{1}$ Anaesthetics, ${ }^{2}$ Pharmacy, University College Hospital, London,

${ }^{3}$ Critical Care \& Anaesthesia, University College London, London, United Kingdom

Objectives: There have been significant changes to the infrastructure of emergency departments (EDs) in the United Kingdom. Introduction of the European Working Time Directive, and initiatives to increase consultant presence in EDs have altered traditional working dynamics. Management of sepsis and evidence, specifically from the military has modified behavior in management of haemorrhage. In light of changes in the way in which care is delivered in EDs, we were keen to investigate prescribing practices for intravenous fluid at University College London Hospital, with approximately 120,000 patient attendances per annum.

Methods: We analysed intravenous fluid (colloids and crystalloids) prescribed in our ED over a seven year period (2006-2013) inclusive. Data was collected from our pharmacy database PIMS and analysed using Excel. Variables including type of fluid and mean volume per patient were analysed. Changes to these variables were compared over the time period. Blood products were excluded from our evaluation.

Results: $0.9 \%$ saline was consistently the most commonly prescribed intravenous fluid in the ED; $70 \%$ (mean), $62.6 \%>75.4 \%$ (range). Other intravenous fluids were used in relatively small amounts, Hartmann's solution and Gelatin being the next most common, comprising $20.7 \%$ and $4.0 \%$ (mean) respectively. Total volume of intravenous fluid prescribed per patient was $451 \mathrm{ml}$ (mean), 400$538 \mathrm{ml}$ (range) over the seven year period.

Conclusion: $0.9 \%$ saline remains the fluid of choice in our ED, despite evidence that crystalloids with high sodium and chloride load place strain on the excretory mechanisms of the kidney and can contribute to hyperchloraemic acidosis. This has been supported by literature endorsing more balanced salt solutions. Given its widespread use in critical care and surgery, we are surprised that balanced crystalloid solutions, are used less frequently in ED. Use of colloid is decreasing and is likely to reflect concerns over the safety of starches and the lack of evidence to support their superiority in the management of sepsis. In line with national recommendations, [1] we advocate ongoing teaching and education in intravenous fluid prescribing practices for all clinicians.

Disclosure of Interest: None Declared. 


\section{PO049}

INCIDENCE OF ACUTE KIDNEY INJURY (AKI)

FOLLOWING ELECTIVE PRIMARY TOTAL HIP REPLACEMENT (THR) IN PATIENTS ON AN ENHANCED RECOVERY PROGRAMME (ERP)

Hari Arunachalam ${ }^{*}{ }^{1}$, Bessie Ayres ${ }^{2}$, Cathryn Eitel $^{1}$

${ }^{1}$ Anaesthetics, ${ }^{2}$ ER Data Analyst, St Richard's Hospital, Western Sussex NHS Foundation Trust, Chichester, United Kingdom

Objectives: ERPs are commonplace in elective primary THR. However, AKI with its associated implications remains a significant postoperative complication with quoted incidences of less than $2 \%$ to $15 \%^{1}$. We sought to determine our incidence of AKI and identify factors associated with increased risk of AKI.

Methods: From January to December 2014, all patients undergoing elective primary THR were enrolled into a protocolised ERP. Demographic, peri- and post-operative data were prospectively collected and analysed using SPSS (v.19). AKI was defined using Kidney Disease Improving Global Outcomes (KDIGO) criteria: serum creatinine increase of $\geq 26.5 \mu \mathrm{mol} / 1$ or $1.5-2$ fold increase from baseline within $48 \mathrm{hrs}$. Factors associated with AKI were determined using multiple logistic regression.

Results: 532 patients (62\% female) underwent THR. 33 patients (6.2\% of total THR, $54 \%$ female) developed AKI. AKI patients had a longer mean LOS (6.06 vs 4.70 days). On considering multiple factors (Table 1), only age was found to have a statistically significant impact on the risk of developing AKI (adjusted odds ratio $1.054,95 \% \mathrm{CI}$ $1.002,1.109, \mathrm{p}<0.05)$.

Factors analysed for association with AKI

\begin{tabular}{|c|c|c|c|c|}
\hline & & $\begin{array}{l}\text { Total THR } \\
\mathrm{n}=532(\%)\end{array}$ & $\begin{array}{l}\text { No AKI } \\
\mathrm{n}=499 \\
(\%)\end{array}$ & $\begin{array}{l}\text { AKI } \\
\mathrm{n}=33 \\
(\%)\end{array}$ \\
\hline \multirow[t]{4}{*}{ Age* } & Under 60 & $67(13)$ & $65(13)$ & $2(6)$ \\
\hline & $60-69$ & $134(25)$ & $130(26)$ & $4(12)$ \\
\hline & $70-79$ & $202(38)$ & $189(38)$ & $13(39)$ \\
\hline & Over 80 & $129(24)$ & $115(23)$ & $14(42)$ \\
\hline \multirow{4}{*}{$\begin{array}{l}\text { Blood Loss } \\
\quad(\mathrm{ml})\end{array}$} & $<500$ & $296(56)$ & $275(55)$ & $21(64)$ \\
\hline & $500-1000$ & $90(17)$ & $87(17)$ & $3(9)$ \\
\hline & $>1000$ & $6(1)$ & $4(1)$ & $2(6)$ \\
\hline & Unknown & $140(26)$ & $133(27)$ & $7(21)$ \\
\hline \multicolumn{2}{|c|}{ NSAIDs postop } & $350(66)$ & $328(66)$ & $22(67)$ \\
\hline \multicolumn{2}{|c|}{ Hypotension postop } & $195(37)$ & $186(37)$ & $9(27)$ \\
\hline \multicolumn{2}{|l|}{ CKD preop } & $28(5)$ & $23(5)$ & $5(15)$ \\
\hline \multicolumn{2}{|l|}{ HTN preop } & $276(52)$ & $254(51)$ & $22(67)$ \\
\hline \multicolumn{2}{|c|}{ Diabetes all types preop } & $64(12)$ & $57(11)$ & $7(21)$ \\
\hline \multicolumn{2}{|l|}{ IHD preop } & $88(17)$ & $81(16)$ & $7(21)$ \\
\hline
\end{tabular}

Conclusion: Our AKI incidence of $6.2 \%$ is well within reported rates and these patients had longer mean LOS. While older patients had an increased risk of developing AKI, other factors such as NSAID use had no statistically significant effect. Further analysis and comparison of THR data prior to the introduction of ERP is required to determine the impact of ERP on the incidence of AKI and its consequent implications.

Disclosure of Interest: None Declared.
PO050

\section{THE EFFECT OF EARLY MOBILIZATION ON POSTOPERATIVE OUTCOMES FOLLOWING THORACIC AND ABDOMINAL SURGERY: A SYSTEMATIC REVIEW}

Tanya Castelino ${ }^{*}$, Julio F. Fiore Jr. ${ }^{1}$, Petru Niculiseanu ${ }^{1}$, Berson Augustin $^{2}$, Liane S. Feldman ${ }^{1}$

${ }^{1}$ General Surgery, ${ }^{2}$ Anesthesia, McGill University, Montreal, Canada

Objectives: The negative effects of prolonged immobilization are well described, and early postoperative mobilization is recommended as a component of enhanced recovery pathways. However there is low evidence regarding the relative contribution of early mobilization to patient recovery. This systematic review aims to summarize the evidence regarding the impact of early mobilization on postoperative outcomes after thoracic and abdominal surgery in comparison to standard care.

Methods: Studies were identified from a comprehensive search of 8 electronic databases. Studies were included if they compared a group of adult patients receiving a specific protocol of early mobilization (with out-of-bed activities starting no later than postoperative day one) to a control group receiving no structured mobilization protocol. Outcomes of interest included complications, length of stay (LOS), pulmonary and gastrointestinal (GI) function, performance-based functional tests, and patient-reported outcomes (PROs).

Results: Six studies in abdominal surgery (3 randomized controlled trials and 3 observational studies) and one study in thoracic surgery (observational) met the inclusion criteria. The overall methodological quality of the studies was low. A wide variety of protocols were employed with some including supervised exercises and others providing education and clear goal-setting. Outcomes reported were variable. In abdominal surgery, two of the four studies evaluating LOS reported a significantly shorter stay in patients who received the mobilization protocol. One study reported faster recovery of GI function. None of the studies reported differences in overall postoperative complications, performance-based tests or PROs. In the study involving thoracic surgery the authors reported that early mobilization was safe, but did not reduce complications or LOS.

Conclusion: Very few comparative studies evaluated the impact of early mobilization on outcomes after abdominal and thoracic surgery. The quality of these studies was generally poor and results were conflicting. Further studies of high methodological quality must be done to contribute evidence about the importance of early mobilization in postoperative care.

Disclosure of Interest: None Declared. 


\section{PO051}

\section{LIPOSOMAL BUPIVACAINE IMPROVES OUTCOMES IN ILEOSTOMY REVERSAL SURGERY AND MAY BE EFFECTIVE IN MORE COMPLEX ENHANCED RECOVERY PROCEDURES}

Aaron Skolnik ${ }^{*}{ }^{1}$, Julie Thacker ${ }^{1}$, Lacey Lee ${ }^{1}$, Timothy Miller ${ }^{1}$, Christopher Mantyh ${ }^{1}$, Tong Gan ${ }^{2}$

${ }^{1}$ Duke University Health System, Durham, ${ }^{2}$ Stony Brook University Hospital, Stony Brook, United States

Objectives: The efficacy of liposomal bupivacaine $\left(\right.$ Exparel $\left.^{\circledR}\right)$, an extendedrelease formulation of the local anesthetic bupivacaine, has been demonstrated in patients undergoing bunionectomy and hemorrhoidectomy. The present study evaluates the efficacy of liposomal bupivacaine (LB) in elective ileostomy reversal surgery.

Methods: Forty-three patients undergoing loop ileostomy closure were treated with liposomal bupivacaine $(266 \mathrm{mg})$ via subcutaneous and suprafascial injection. Primary outcomes included total opioid consumption during the first 24 hours postoperatively and for the duration of the patient's hospitalization. Secondary outcomes included: length of hospital stay, maximum pain score (010 on the Numerical Rating Scale [NRS]), postoperative nausea, and administration of antiemetic medication. The data were compared retrospectively with a similar cohort $(\mathrm{n}=35)$ who received $10 \mathrm{~mL}$ of plain bupivacaine $0.25 \%$ for analgesia. Student's t-test and two-proportion z-test were used for statistical analysis. A p-value $<0.05$ was considered statistically significant. Results:

\begin{tabular}{|c|c|c|c|}
\hline & $\begin{array}{l}\text { Liposomal } \\
\text { bupivacaine } \\
\text { group } \\
(\mathrm{n}=43)\end{array}$ & $\begin{array}{l}\text { Control } \\
\text { group } \\
\text { (bupivacaine } \\
\mathrm{HCl}) \\
(\mathrm{n}=35)\end{array}$ & P-value \\
\hline $\begin{array}{l}24 \text { h postoperative opioid } \\
\text { consumption, IV morphine- } \\
\text { equivalents (mg), mean (SD) }\end{array}$ & $15.2(15.1)$ & $29.0(28.0)$ & $P=0.01^{*}$ \\
\hline During entire hospitalization & $22.4(22.3)$ & $79.9(146.1)$ & $\mathrm{P}=0.03^{*}$ \\
\hline Length of stay (days), mean (SD) & $1.3(1.2)$ & $3.2(3.2)$ & $\mathrm{P}=0.002$ \\
\hline $\begin{array}{l}24 \text { h postoperative maximum pain } \\
\text { score }(0-10 \mathrm{NRS}), \text { mean }(\mathrm{SD})\end{array}$ & $6.3(3.4)$ & $6.9(2.4)$ & $P=0.39$ \\
\hline$>24 \mathrm{~h}$ postop & $4.7(3.5)$ & $7.0(2.7)$ & $\mathrm{P}=0.009^{*}$ \\
\hline $\begin{array}{l}\text { Number of patients reporting } \\
\text { nausea during entire } \\
\text { hospitalization }\end{array}$ & $13(30.2 \%)$ & $15(42.9 \%)$ & $\mathrm{P}=0.25$ \\
\hline $\begin{array}{l}\text { Number of patients receiving } \\
\text { antiemetic medication during } \\
\text { entire hospitalization }\end{array}$ & $16(37.2 \%)$ & $17(48.6 \%)$ & $\mathrm{P}=0.31$ \\
\hline Cost of hospitalization, mean (SD) & $\$ 19,888.10$ & & \\
\hline$(\$ 3,516.70)$ & $\$ 25,130.60$ & & \\
\hline$(\$ 11,956.50)$ & $\mathrm{P}=0.02 *$ & & \\
\hline
\end{tabular}

Conclusion: For patients undergoing ileostomy reversal, liposomal bupivacaine is associated with decreased opioid consumption, pain scores, length of stay, and cost. If added to an enhanced recovery program, liposomal bupivacaine could impart similar outcome improvements in more complex surgeries with comparable incision length (e.g. laparoscopic colectomy).

Disclosure of Interest: A. Skolnik: None Declared, J. Thacker: None Declared, L. Lee: None Declared, T. Miller: None Declared, C. Mantyh: None Declared, T. Gan Grant / Research support from: Pacira Pharmaceuticals, Inc.

\section{PO052}

\section{IMPLEMENTATION OF ENHANCED RECOVERY AFTER SURGERY IN COLORECTAL PATIENTS: THE IMPACT ON NUTRITION CARE}

Lisa Martin $^{1}$, Marlis Atkins ${ }^{2}$, Carlotta Basualdo-Hammond ${ }^{2}$, Lawrence Kiyang ${ }^{2}$, Gregg Nelson ${ }^{*}{ }^{3}$, Leah Gramlich ${ }^{2}$

${ }^{1}$ University of Alberta, ${ }^{2}$ Alberta Health Services, Edmonton, ${ }^{3}$ University of Calgary, Calgary, Canada

Objectives: ERAS improves nutritional care of surgical patients by monitoring adherence to guidelines. ERAS guidelines include pre-operative (PO) malnutrition screening, reduced length of PO fasting combined with carbohydrate loading, and early PO oral intake. Prior to ERAS, these nutrition interventions were not standard of care for surgical patients in Alberta Health Services, Canada. This study describes nutrition risk in elective colorectal surgical patients, compliance with nutrition care interventions after ERAS implementation, and to identifies opportunities for improvement with ERAS nutrition care interventions.

Methods: ERAS was implemented in elective colorectal surgical patients in 6 hospitals in Alberta, Canada. Data related to nutrition care (malnutrition screening, carbohydrate loading, and early oral intake) were analyzed for compliance and completeness of data reporting. The Malnutrition Screening Tool (MST) assessed risk of malnutrition, carbohydrate loading was assessed using 'yes' or 'no', early oral intake was assessed by days to tolerating solid food, and energy intake from ONS on PO days 0-3.

Results: 708 ERAS patients were included. $71 \%$ of patients were screened using MST: $2 \%$ high risk, $14 \%$ moderate risk, and $84 \%$ at low risk of malnutrition; $9 \%$ had missing data. Carbohydrate loading occurred in $52 \%$ of patients; $8 \%$ had missing data. Data for early oral intake are summarized in Table 1; the mean energy intake per day is lower than the daily target of 600 Kcal.

Table 1 Summary of post-operative energy intake from oral nutrition supplementation (ONS)

\begin{tabular}{lcccc}
\hline & POD 0 & POD 1 & POD 2 & POD 3 \\
\hline $\begin{array}{l}\text { Total Kcal } \\
(\text { mean } \pm \text { S.D. })\end{array}$ & $\begin{array}{c}133.1 \pm \\
147.1\end{array}$ & $\begin{array}{c}334 \pm \\
243.9\end{array}$ & $\begin{array}{c}307 \pm \\
246.7\end{array}$ & $\begin{array}{c}228 \pm \\
235.21\end{array}$ \\
$\begin{array}{l}\text { ONS taken } \\
\quad \% \text { reported })\end{array}$ & 37 & 53 & 49 & 37 \\
$\begin{array}{l}\text { ONS not taken } \\
(\% \text { reported })\end{array}$ & 31 & 19 & 21 & 24 \\
\begin{tabular}{l} 
Missing data $(\%$ total $)$ \\
\hline
\end{tabular} & 32 & 28 & 30 & 39 \\
\hline
\end{tabular}

$P O D=$ Post-operative day

Conclusion: ERAS supported the adoption of standard nutrition care for elective colorectal surgery patients. Improvements in nutrition care have occurred, however gaps (e.g. poor compliance, missing documentation) exist 1 year after ERAS implementation. Emphasize on the importance of nutrition care and documentation to monitor progress is needed. Barriers to compliance will be improved through team collaboration, and strategies at the patient, provider and system level.

Disclosure of Interest: None Declared. 


\section{PO053}

\section{ERAS PROTOCOLS FOR URGENT COLORECTAL SURGERY}

Riza Haldun Gundogdu ${ }^{*}{ }^{1}$, Soner Akbaba ${ }^{1}$, Ogtay Nasrullayev ${ }^{1}$, Pamir E. Ersoy ${ }^{1}$, Omer Yazicioglu ${ }^{1}$, Bahadir O. Bozkirli ${ }^{2}$

${ }^{1}$ General Surgery, Ataturk Training and Research Hospital, ${ }^{2}$ General Surgery, Ankara Training and Research Hospital, Ankara, Turkey

Objectives: The use of enhanced recovery (ERAS) protocols in colorectal surgery allows a significant reduction of postoperative complications and hospital stay. However, these principles have not yet been reported for urgent operations. We aimed to assess our experience with urgent colorectal operations performed within an established ERAS program.

Methods: We included 102 colonic resections, which were performed according to ERAS protocols between January 2010 and December 2014. Urgent operations were compared with the elective procedures with regards to baseline characteristics, compliance with ERAS items, and outcome.

Results: Patients $(n=23)$ requiring urgent colonic resection were included and compared with patients undergoing elective colectomy $(n=79)$. Overall compliance with the protocol was $69.5 \%$ for the urgent compared with $86 \%$ for the elective procedures $(\mathrm{p}<0.05)$. But, the intra-operative compliance was $79 \%$ versus $84 \%(\mathrm{p}>0.05)$. Overall, 15 urgent cases (65.2\%) and 41 elective patients $(51.8 \%)$ developed postoperative complications $(\mathrm{p}>0.05)$. Median postoperative length of stay was 10 days in the urgent setting compared with 7 days in the elective setting $(\mathrm{p}<0.05)$.

Conclusion: Many of the ERAS items can also be applied to urgent colonic operations without significant adverse effect. ERAS protocols can therefore be considered for patients undergoing urgent colorectal surgery.

Disclosure of Interest: None Declared.

\section{PO054}

\section{CURRENT STATE OF NUTRITIONAL ERAS ELEMENTS IN} SPAIN. A NATIONAL SURVEY

Javier Ripollés Melchor ${ }^{1}$, Rubén Casans Francés ${ }^{2}$, Eugenio Martínez Hurtado $^{1}$, Alfredo Abad Gurumeta ${ }^{3}$, Alejandro Suarez de la Rica ${ }^{3}$, José Manuel Ramírez ${ }^{*}$, José María Calvo Vecino ${ }^{1}$ on behalf of GERM. Grupo Español de Rehabilitación Multimodal

${ }^{1}$ Anesthesia and Critical Care, Hospital Universitario Infanta Leonor, Madrid, ${ }^{2}$ Anesthesia and Critical Care, Hospital Universitario Lozano Blesa, Zaragoza, ${ }^{3}$ Anesthesia and Critical Care, Hospital Universitario La Paz, Madrid, ${ }^{4}$ General Surgery, Hospital Universitario Lozano Blesa, Zaragoza, Spain

Objectives: The aim of this study was to survey the interest, the knowledge of ERAS protocols, and compliance with perioperative care items.Particularly those items related to nutrition.

Methods: Free access survey was conducted professionals, hosted on the websites of the Spanish society of anesthesiology and critical care (SEDAR); Spanish association of surgeons (AEC) and Spanish society of enteral and parenteral nutrition (SENPE) and ERAS Spain (GERM) between September 2014 and December 2014.

Results: Survey was answered by 256 professionals, (anesthetists $43 \%$, general surgeons $44 \%$ ) from 110 hospitals. 58\% had experience in ERAS protocols, $85 \%$ knew ERAS protocols, whereas only $52 \%$ knew the ERAS recommendations $71 \%$ of respondents say ERAS protocols are performed in their hospitals, mainly in colorectal $(68 \%)$ laparoscopic surgery $(55 \%)$ and based on ERAS recommendations in. $56 \%$ think there is a good implementation of ERAS protocols, while $56 \%$ think that are satisfactory. $94 \%$ are interested in development of multidisciplinary Spanish guidelines; and, with the development of these, $87 \%$ start to perform these. $47 \%$ perform preoperative nutritional assessment, although there is no universal malnutrition screening method (36\%); and is based mainly on weight loss (18\%). Usually there is not a protocol for referral to dietician (35\%), neither preoperative nutritional support is indicated in cases of severe malnutrition (44\%); and, when performed, only in 39\% of cases is performed with immunomodulating formulations. In a minority of cases preoperative loading with carbohydrate drinks is carried out $(44 \%)$. Nasogastric tube and drainage are avoided (64\%), prophylaxis of postoperative nausea and vomiting (57\%), goal directed fluid therapy (57\%) are performed. In most cases early feeding (73\%) is performed and in cases of malnutrition postoperative nutritional support is carried out $(69 \%)$. The leading causes of protocol failure are postoperative nausea and vomiting (31\%) and ileus (40\%).

Conclusion: Conducting fast track protocols is known in Spain, however, not seem to exist a consensus, nor are performed according to guidelines. Exists a deficit in the perioperative nutritional management.

Disclosure of Interest: None Declared. 


\section{PO055}

\section{USE OF AN INTRAVENOUS ELASTOMERIC PUMP AS POSTOPERATIVE ANALGESIA IN A COLORECTAL SURGERY ERAS PROGRAM. ONE FURTHER STEP TOWARDS MINIMAL INVASION}

Marta Cuadrado $^{*}{ }^{1}$, Ignasi Camps ${ }^{1}$

${ }^{1}$ General and Digestive Surgery, Hospital Universitari Germans Trias i Pujol, Badalona (Barcelona), Spain

Objectives: Achieve effective analgesia with a much less invasive technique.

Methods: Since January $1^{\text {st }} 2014$, we introduced in all elective colorectal surgery ERAS program the use of an intravenous elastomeric pump (EP) (Dexketoprofen + tramadol + droperidol) as postoperative analgesia. This period is compared with a 2013 retrospective series, in which analgesia was performed with epidural catheter (EC) PCA pump (fentanyl + bupivacaine). Both methods of analgesic infusion were used during the first $36 \mathrm{~h}$ and after were subsequently replaced by conventional IV analgesia (metamizol + paracetamol). We have analyzed postoperative pain scores, tolerate regular diet, complications and length of stay.

Results: There are no significant differences in the type of patient, surgical technique, ASA score and surgical complications. There is also no difference in analgesia achieved and toleration of a regular diet. However, while all patients with EP can get up from bed at 6 hours after surgery, we found that almost $5 \%$ of patients with CE have some kind of functional impairment in the lower limbs that difficult early mobilization. The average stay is 24 hours less in 2014 (7.2 days vs. 6'2).

Conclusion: In the absence of more conclusive studies, we believe that the elastomeric pump is a good analgesic technique, much less invasive, avoiding anxiety, risk of patient complications and preoperative less time consuming.

Disclosure of Interest: None Declared.
PO056

\section{POSTOPERATIVE 4 DAY DISCHARGE OF ROBOTIC GASTRECTOMY FOR GASTRIC CANCER PATIENTS APPLIED WITH ERAS PROGRAM, A PROSPECTIVE} CLINICAL TRIAL

Shu Zhang ${ }^{*}{ }^{1}$, Gang Wang ${ }^{1}$, Zhi-Wei Jiang ${ }^{1}$, Kun Zhao ${ }^{1}$, Jie-Shou Li ${ }^{1}$

${ }^{1}$ Research Institute of General Surgery, Jinling Hospital, Nanjing University, Nanjing, China

Objectives: The aim of this study was to determine the influence and safety of robotic surgery applied with ERAS protocol in oncological gastrectomy on early outcomes especially for patients discharged from Hospital within 4 days.

Methods: We Prospective analyzed 40 patients (10 female and 30 male) with gastric cancer who underwent robotic gastrectomy with standard D2 lymphnodes resection between Jun 2014 and Dec 2014. Perioperative care was based on ERAS principles. The postoperation complications and Length of hospital stay, postoperative morbidity and mortality were analyzed as well as the 30 -days readmission rates. A series of clinical and laboratory examination were also recorded an improvement from postoperation day 1 (POD1) to postoperation day 3 (POD3), such as the SPO2, Heart Rate (HR), C-reactive protein (CRP), hemoglobin (HB), hemoglobin (ALB), prealbumin (PA), transferrin $(\mathrm{TF})$.and also an comparison was conducted between $<4 \mathrm{~d}$ Group to $>4 \mathrm{~d}$ Group in VAS, sleep time and postoperation walk steps on POD1, POD2 and POD3.

Results: There was no conversion to open surgery in the gastrectomy group. All patients were encouraged to walk on the morning of POD1. Oral glucose fluids were introduced on POD1. Full diet was started on the regain of bowl movement in all patients. No postoperative complication requiring reoperation was noted. Not only is there an improvement from postoperation day 1 (POD1) to postoperation day 3 (POD3), but also an improvement in the comparison of $<4 \mathrm{~d}$ Group to $>4 \mathrm{~d}$ Group, in laboratory findings and clinical findings such as the SPO2, Heart Rate (HR), C-reactive protein (CRP), hemoglobin (HB), hemoglobin (ALB), prealbumin (PA), transferrin (TF). The $<4 \mathrm{~d}$ Group is of the same quality and safety concerning that the statistics shows no significant difference between in the two group. The $<4 \mathrm{~d}$ Group is of no more risk of postoperative complications or 30 day's re-admission.

Conclusion: The Robotic Gastrectomy with Standard D2 Lymphnodes Resection with ERAS protocol can improve postoperative care quality, shorten of hospital stay, and might be a opitmal choice for gastric cancer patients.

Disclosure of Interest: None Declared. 


\section{PO057}

\section{IMPLEMENTING ERAS WITHIN THE INTEGRATED HEALTHCARE DELIVERY SYSTEM OF KAISER PERMANENTE NORTHERN CALIFORNIA (KPNC)}

Mengfei Huang ${ }^{*}{ }^{1}$, Alexander Mustille ${ }^{1}$, Efren Rosas ${ }^{1}$, Judith Hwang ${ }^{1}$, Vincent Liu ${ }^{1}$, Anne Foss-Durant ${ }^{1}$, Stephen Parodi ${ }^{1}$

${ }^{1}$ Kaiser Permanente, Oakland, United States

Objectives: KPNC aimed to improve peri and postoperative patient outcomes through implementation of an ERAS program across 21 medical centers. The scope of this program included elective and emergent colorectal surgery and hip fractures.

Methods: A KPNC team developed ERAS protocols after an exhaustive literature review and consideration of internal operational requirements. The protocols were piloted at 2 medical centers with a plan for continuous performance improvement and refinement of the workflows. Areas assessed included: multimodal analgesia, early mobility, and optimal nutrition. A measurement strategy retrieved from the electronic medical record (EMR) and standardized using NSQIP guidelines consisted of: (1) Implementation metrics; (2) Process metrics; (3) Patient outcomes. A dashboard was provided to each facility's local ERAS implementation teams to maximize interdisciplinary collaboration and leadership alignment.

Results: Comprehensive KPNC ERAS implementation began July 2014 with a regional educational summit attended by 385 frontline staff and local leadership. As of November 2014, all 21 medical centers had implemented the colorectal and hip fracture pathways, impacting the workflow of several hundred colorectal surgeons, anesthesiologists, and orthopedic surgeons, several thousand nurses, and many other groups, including pharmacists, emergency room physicians, physical therapists, nutritionists. 13 ordersets were implemented within the EMR to provide real-time decision support. In this early phase of implementation, data from $>1200$ patients demonstrates significantly decreased length of stay as well as increases in early feeding and ambulation. Ongoing qualitative patient and staff assessments align with these early findings.

\begin{tabular}{ll}
\hline Process Metrics & $\begin{array}{l}\text { Outcome/Balancing } \\
\text { Metrics }\end{array}$ \\
\hline Last Liquids \& Solids & Length of Stay \\
Multimodal Analgesia & ED to Surgery Time \\
Morphine Equivalents & Mortality \\
Early Feeding \& Ambulation & Readmissions \\
& Pneumonia \\
& UTI \\
& Transfusion \\
& DVT/PE \\
\hline
\end{tabular}

Conclusion: Through the collaborative efforts of thousands of clinicians and staff, the KPNC ERAS program was implemented across 21 hospitals in 9 months from pilot to regionwide implementation, rapidly analyzing new interdisciplinary collaboration and significantly improving patient care.

Disclosure of Interest: None Declared.

\section{PO058}

\section{RISK REDUCTION IN COLORECTAL SURGERY: PERIOPERATIVE MANAGEMENT OF CARDIOVASCULAR MEDICINES}

Matthew T. Royds ${ }^{*}{ }^{1}$, Jonathan Wedgwood ${ }^{1}$, Angie Balfour ${ }^{2}$, Hugh Patterson $^{2}$

${ }^{1}$ Anaesthesia, ${ }^{2}$ Colorectal Surgery, Western General Hospital, Edinburgh, United Kingdom

Objectives: Withdrawal of certain cardiovascular medicines, particularly aspirin, statins and beta-blockers may be associated with increased risk of adverse cardiac events (ACE) in the perioperative period $^{1}$.

We evaluated the perioperative management of these medications in patients suffering ACE after elective colorectal surgery, with the aim of identifying potential areas for quality improvement.

Methods: Patients suffering ACE after elective colorectal surgery were identified using the Lothian Colorectal Database. This contains prospectively collected data on all patients undergoing colorectal surgery in our hospital. Data were collected between January 2010 and May 2013. Notes were reviewed for these patients to establish whether they were usually on aspirin, statin, or beta-blocker and whether these medications had been administered or withheld in the perioperative period.

Results: There were 1504 patients in the Colorectal Database of whom $102(6.8 \%)$ experienced postoperative ACE. Details of the perioperative management of aspirin, statins and beta-blockers in the subgroup suffering ACE are shown in the table.

\begin{tabular}{llcll}
\hline Medication & $\begin{array}{l}\text { Number of } \\
\text { patients on } \\
\text { medication }\end{array}$ & $\begin{array}{l}\text { Medication } \\
\text { administered }\end{array}$ & $\begin{array}{l}\text { Medication } \\
\text { omitted }\end{array}$ & $\begin{array}{l}\text { Mean } \\
\text { number } \\
\text { days } \\
\text { omitted }\end{array}$ \\
\hline Aspirin & 36 & $9(25 \%)$ & $27(75 \%)$ & 4 \\
Statin & 45 & $24(53 \%)$ & $21(47 \%)$ & 3 \\
Beta-blocker & 30 & $16(53 \%)$ & $14(47 \%)$ & 3 \\
\hline
\end{tabular}

Conclusion: We have identified that a significant proportion of patients suffering ACE after elective colorectal surgery in our institution had their chronic aspirin, statin or beta-blocker medication withheld in the postoperative period. Current evidence suggests that acute withdrawal of these medications in the perioperative period may be associated with an increased risk of ACE, and therefore this represents a potential area for improvement in our current practice. In order to address this, we have developed a simple handbook that has now been introduced at ward level. Reducing perioperative risk in colorectal surgery: An introductory guide for all staff provides accessible evidence-based perioperative guidance for nurses and junior doctors, including on the management of chronic cardiovascular medication. We hope that the introduction of this handbook will reduce harmful variation and encourage best evidence-based practice.

Disclosure of Interest: None Declared. 


\section{PO059}

\section{ORGANIZATIONAL CULTURE CHANGES RESULTS IN IMPROVEMENT IN PATIENT-CENTERED OUTCOMES: IMPLEMENTATION OF AN INTEGRATED RECOVERY PLAN FOR SURGICAL PATIENTS}

Deborah Hobson $^{*}{ }^{1}$, Elizabeth Wick ${ }^{1}$, Daniel Galante ${ }^{1}$, Andrew Benson $^{2}$, Ken Lee ${ }^{3}$, Renee Demski ${ }^{3}$, Peter Pronovost ${ }^{3}$, Christopher $\mathrm{Wu}^{2}$

${ }^{1}$ Department of Surgery, ${ }^{2}$ Department of Anesthesia and Critical Care Medicine, ${ }^{3}$ Armstrong Institute for Patient Safety and Quality, Johns Hopkins University, Baltimore, United States

Objectives: The goal of quality improvement is to partner with patients, their loved ones and others to end preventable harm, improve patient outcomes and experience, and eliminate waste yet few improvement programs have successfully worked on all of these elements in concert. Improvement programs should aspire to address all of these elements in an integrated, holistic approach.

Methods: We designed a study to evaluate the impact of the implementation of an integrated recovery pathway to improve patient outcomes, value and experience in colorectal surgery. Eleven months of pre- and post-pathway intervention outcomes (length of stay (LOS), surgical site infection (SSI), venous thromboembolism (VTE) and urinary tract infection (UTI), patient experience and variable direct costs) were compared. Pathway implementation expanded on the pre-existing hospital comprehensive unit based safety program infrastructure and used a trust based accountability model at each level from senior leaders to frontline staff to support the initiative.

Results: 310 patients underwent surgery in the baseline period, the mean LOS was 7 days and the mean SSI rate was 18.8\%. 330 patients underwent surgery on the pathway. After commencing the pathway, the LOS was 5 days and the SSI rate was $7.3 \%$ for the subsequent 11 months. Concurrently, improvement in the patients experience was observed as measured through the Hospital Consumer Assessment of Healthcare Providers and Systems (HCAHPS) survey. The interventions included enhanced pre-operative education, mechanical bowel preparation with oral antibiotics, chlorhexidine bathing, multimodal analgesia, restricted intravenous fluids protocol, early mobilization and resumption of oral intake.

Conclusion: An organizational model of collaborative leadership combined with trust based accountability model that included both senior hospital leadership and frontline providers provided an enabling structure to rapidly implement an integrated recovery pathway and quickly improve the outcomes, value and experience of patients undergoing colorectal surgery. The study findings have significant implications for spreading surgical quality improvement work.
PO060

\section{WHAT EFFECT HAS THE INTRODUCTION OF ERAS HAD ON LENGTH OF STAY AFTER TOTAL HIP AND KNEE REPLACEMENT IN THE SOUTH WEST REGION OF ENGLAND?}

\author{
Thomas W. Wainwright ${ }^{*}{ }^{1}$, Robert G. Middleton ${ }^{1}$ \\ ${ }^{1}$ Orthopaedic Research Institute, Bournemouth University, \\ Bournemouth, United Kingdom
}

Objectives: To determine the effect or ERAS introduction on aggregated mean length of stay (LOS) for hip and knee replacement across 17 hospitals in the South West region of England.

Methods: Dr Foster software (Practice and Provider Monitor tool) was used to interrogate Hospital Episode Statistic data. Data from Q42007 to Q4-2014 was retrieved for elective hip and knee replacement spells from hospitals within the South West region of England. Aggregated mean LOS was then calculated per quarter.

Results: 56,074 knee replacements and 58,399 hip replacements performed in 17 hospitals were included in the analysis. Over the time period from Q4-2007 to Q4-2014, across the region mean LOS reduced by 2.1 days from 7.2 days to 5.1 days for hip replacement, and by 1.7 days from 6.9 days to 5.2 days for knee replacement. Within this reduction of total LOS, over the same time period the pre-operative LOS reduced by 0.4 days ( 0.5 to 0.1 for hip replacement, and 0.4 to 0.0 for knee replacement).

Conclusion: The introduction of ERAS within the UK has helped to reduce mean LOS for hip and knee replacement in the South West of England over the last 7 years. However, when aggregated data with no exclusions is analysed across the region there is still significant scope for further reduction in LOS when the results of international exemplar ERAS sites are compared. The data may suggest that ERAS adoption has not been as complete as previously thought and reported. This highlights the very important need for further high profile ERAS awareness raising, and on-going support to help hospitals successfully implement ERAS for all of their hip and knee replacement patients.

Disclosure of Interest: None Declared.

Disclosure of Interest: None Declared. 


\section{PO061}

\section{IMPLEMENTATION OF AN ENHANCED RECOVERY AFTER GYNECOLOGIC ONCOLOGY SURGERY PATHWAY: IMPROVING COMPLIANCE WITH KEY ELEMENTS OF PREOPERATIVE CARE}

Javier D. Lasala ${ }^{*}{ }^{1}$, Larissa A. Meyer ${ }^{2}$, Alpa M. Nick ${ }^{2}$, Pedro Ramirez ${ }^{2}$, Gabriel E. Mena ${ }^{1}$, Juan P. Cata ${ }^{1}$, Vijay Gottumukkala ${ }^{1}$, Piotr Kwater ${ }^{1}$, Shannon Hancher-Hodges ${ }^{1}$, Katherine Cain ${ }^{3}$, Melinda Harris $^{2}$, Lindsey Palmer ${ }^{2}$

${ }^{1}$ Anesthesiology, ${ }^{2}$ Gynecologic Oncology and Reproductive Medicine, ${ }^{3}$ Pharmacy Clinical Programs, University of Texas MD Anderson Cancer Center, Houston, United States

Objectives: To describe a process change and evaluate compliance with use of preventive analgesia and deep venous thromboembolic (DVT) prophylaxis in an ERP pathway. One of the key elements of the Enhanced Recovery after Gynecologic Surgery program at MD Anderson Cancer Center is preventive analgesia and perioperative multi-modal opioid sparing strategies.

Methods: The ERP at MD Anderson was initiated in the Department of Gynecologic Oncology in November 2014. We used the following medications for preventive analgesia; pregabalin (neuropathic pain), celecoxib (non-steroidal anti-inflammatory), and tramadol (mu-opioid receptor, synthetic opioid). To assure compliance an order set was developed to include these medications in all patients undergoing gynecologic surgery provided there were no contraindications. All patients were also written in the order set to receive subcutaneous heparin 5,000 units subcutaneously administered on arrival to holding area. The order set is completed at the time of surgical posting by a member of the gynecologic oncology team. Medications are administered upon arrival in the preoperative holding area by the nursing staff. Training on the new order set was provided prior to ERP rollout in November to members of the gynecologic oncology team as well as to the nurses in the preoperative holding area. To improve adherence to the process change, a repeat training was performed for the clinic staff 6 weeks after implementation.

Results: A total of 60 patients were included in the analysis. Since the implementation of our program and the availability of the order set our compliance for pre-emptive analgesia and subcutaneous heparin orders in the surgical posting period has been $87 \%$ for the first 60 patients (52/60). All 100\% of these patients (60/60) had the order set complete by the time they arrived in holding, as anesthesiologists verify the order set being present before midnight of the day of surgery, if not present it is ordered at that time.

Conclusion: Creation of specific order sets can be helpful to improve compliance with ERP. However, as with any new process change, audits of process flow can identify areas for improvement.

Disclosure of Interest: None Declared.

\section{PO062}

\section{PERIOPERATIVE ALLOGENEIC BLOOD TRANSFUSION IN ELECTIVE COLORECTAL SURGERY}

Matthew T. Royds $^{*}{ }^{1}$, Jonathan Wedgwood ${ }^{1}$, Clair Baldie ${ }^{1}$

${ }^{1}$ Anaesthesia, Western General Hospital, Edinburgh, United Kingdom

Objectives: Perioperative allogeneic blood transfusion (ABT) is independently associated with adverse outcomes in terms of mortality, complications and cancer recurrence following surgery ${ }^{1}$. We have examined transfusion practice in our elective colorectal surgical population in order to identify potential areas for quality improvement.

Methods: We retrospectively identified all patients undergoing elective colorectal resection in our institution during 2013 using theatre management software. Cross-referencing with Blood Transfusion Service (BTS) records we identified all cases receiving ABT. Where ABT occurred we noted the timing relative to surgery, the number of units transfused, and the post-transfusion haemoglobin (Hb) level.

Results: There were 550 elective colorectal resections undertaken in the study period. Overall $83(15 \%)$ received ABT. We classified ABT as minor if transfusion was two units or less of packed red-cells (PRC). Intra-operative ABT occurred in 54 (10\%) patients, of which $34(6 \%)$ were minor transfusions. Median (IQ) post-transfusion $\mathrm{Hb}$ was $105(97,112) \mathrm{g} / \mathrm{l}$ in the intra-operative subgroup. Post-operative $A B T$ (within 48 hours of surgery) occurred in $29(5 \%)$ patients of which 24 (4\%) were minor. Median (IQ) post-transfusion Hb was 98 $(92,105) \mathrm{g} / \mathrm{l}$ in the post-operative subgroup. Median (IQ) pre-transfusion $\mathrm{Hb}$ measured prior to post-operative ABT was $79(76,86) \mathrm{g} / 1$. Conclusion: The ABT rate is $15 \%$ in our cohort. A significant proportion of this comprises minor transfusions of two units or less PRC. There appears to be a trend toward more liberal transfusion thresholds in our current practice, with the majority of cases having post-transfusion $\mathrm{Hb}>100 \mathrm{~g} / \mathrm{l}$. Given the adverse effects of perioperative ABT on outcome, and the lack of evidence to support a liberal versus restrictive transfusion straregy ${ }^{2}$, this could represent an area for potentially improved evidence-based practice. We believe that the introduction of a more restrictive transfusion policy, and in particular avoiding minor (and thus possibly discretionary) transfusions, coupled with preoperative anaemia correction could lead to significant risk-reduction in our patient population.

Disclosure of Interest: None Declared. 


\section{PO063}

\section{ENHANCED RECOVERY AFTER SURGERY PROVIDES HIGH LEVEL OF PATIENT SATISFACTION IN LAPAROSCOPIC AND OPEN GYNAECOLOGICAL SURGERY}

Joanne A. L. Lee ${ }^{*}{ }^{1}$, Arun Nair ${ }^{2}$, Victoria White ${ }^{1}$, Catherine Brocklehurst $^{1}$, Ian Lyons ${ }^{2}$, Sian Davies ${ }^{2}$, Martyn Traves ${ }^{2}$, Anish Bali ${ }^{1}$

${ }^{1}$ Obstetrics and Gynaecology, ${ }^{2}$ Anaesthesia, Royal Derby Hospital, Derby, United Kingdom

Objectives: Enhanced recovery after surgery (ERAS) has shown to improve patient care within gynaecology. No previous studies have compared patient's recovery and satisfaction within ERAS program after undergoing major gyneaecological laparoscopic or open surgery. This prospective study investigates if there is a difference in patient's experience in these two groups.

Methods: Consecutive cases undergoing surgery within ERAS program were included from April to September 2014. Vulva and pelvic floor surgery were excluded. We examined the patient's ability to achieve early oral intake, mobilisation and their satisfaction with pain and nausea control, hospital stay and care provided. Questionnaires were given on admission and returned prior to discharge.

Results: There were 263 cases with $48 \%$ having a total laparoscopic hysterectomy and $52 \%$ a laparotomy for simple hysterectomy, complex surgery or cytoreduction with or without bowel resection. 35\% were oncology cases and $65 \%$ benign. $54 \%$ of patients responded $(n=144)$. Half were performed by laparoscopy the remainder by open procedure. In recovery there was better pain and nausea control in the laparoscopic group (LG) with $80 \%$ reporting no or mild pain and nausea versus $54 \%$ in the open group (OG). By day 1 post-op, over $90 \%$ in both groups (LG \& OG) had had oral fluids and eaten solid food demonstrating compliance with ERAS parameters with no significant difference. Majority of patients (76\% LG and 82\% OG) had seen the physiotherapist and achieved mobilization to 60 meters by day 1 with similar percentages sitting in a chair over 2 hrs. There was high patient satisfaction with $73 \%$ satisfied with pain control, $96 \%$ felt they were treated with dignity, $89 \%$ felt informed of their treatment and $86 \%$ felt involved in their decision of care. $98 \%$ patients were either very satisfied or satisfied with the care received from the healthcare team.

Conclusion: Overall, patient's experience and satisfaction between laparoscopic and open groups is high. The aspect of care that can be improved is the pain and nausea control in the immediate postoperative care of the open group.

Disclosure of Interest: None Declared.
PO064

\section{WHAT ISSUES MUST WE ADDRESS IF WE ARE TO FURTHER ACCELERATE RECOVERY AND SHORTEN LENGTH OF STAY IN TOTAL HIP AND KNEE REPLACEMENT ERAS?}

Thomas W. Wainwright ${ }^{*}$, David A. McDonald ${ }^{2}$

${ }^{1}$ Orthopaedic Research Institute, Bournemouth University, Bournemouth, ${ }^{2}$ Directorate of Health Workforce and Performance, Scottish Government, Glasgow, United Kingdom

Objectives: UK ERAS pathways are well established but LOS is still longer than international exemplar sites. This survey asked staff what they felt were the issues that needed to be addressed in order to reduce LOS further.

Methods: Attendees at a multi-disciplinary meeting (Enhanced Recovery in Orthopaedic Surgery Conference, Glasgow, Scotland, 20/ $01 / 15$ ) were surveyed.

Results: 58 respondents from 23 hospitals in England, Scotland, and Ireland, were made up of Nurses (27), Physiotherapists (18), Anaesthetists (7), Occupational Therapists (3), Surgeons (2), and a Pharmacist. 54 respondents had 2 or more years' experience working ERAS. The staff felt that the most important factors for a short LOS within their own hospitals were; providing effective pre-operative patient education and expectation management (45), ensuring effective and adequate post-operative analgesia (41), and ensuring that patients were mobilised early (20). 18 respondents cited the importance of multidisciplinary team working, and 10 cited the importance of improved therapy access. When the staff were asked which issues they felt they needed to improve within their own hospitals to further reduce LOS, 35 felt that better post-operative analgesia was required and 29 felt better pre-operative patient education and expectation management was required. There were 3 other major themes, managing the side effects of anaesthesia ( 22 felt that dizziness, nausea and vomiting needed to be addressed), facilitating early mobilisation ( 25 thought that more physiotherapy access and earlier mobilisation was needed), and ensuring a smooth discharge process (13 thought that better social and community support was required, and 12 highlighted the need for better planning).

Conclusion: Experienced ERAS staff cited pre-operative patient education and post-operative analgesia most frequently as key factors helping to reduce LOS, and also identified these two steps as the ones needing to be improved. Managing the side effects of anaesthesia, improved physiotherapy access, and greater social and community support post discharge were also deemed important.

Disclosure of Interest: None Declared. 


\section{PO065}

\section{BILATERAL INTRAPLEURAL REGIONAL ANAESTHESIA FOR PAIN CONTROL AND ENHANCED RECOVERY FOLLOWING BREAST SURGERY}

Tomasz Nikodemski ${ }^{*}$, Katarzyna Ostrowska-Clark ${ }^{2}$, Jeremy Clark ${ }^{3}$, Arkadiusz Kazimierczak ${ }^{3}$, Leszek Sagan ${ }^{3}$, Rafał Rojek ${ }^{3}$

${ }^{1}$ Centrum Medyczne, Dobra, ${ }^{2}$ Medimel, ${ }^{3}$ Pomeranian Medical University, Szczecin, Poland

Objectives: Non-opioid based pain treatment, is an essential component of enhanced recovery after surgery (ERAS) protocols. The technique of intrapleural analgesia was described 30 years ago, but we have found no publication concerning application of this method for bilateral breast surgery in the literature.

Methods: A case series of 121 patients that underwent either breast augmentation (Group A, $\mathrm{n}=96$ ) or reduction mammoplasty (Group $\mathrm{R}, \mathrm{n}=25$ ) are presented. All patients were given bilateral intrapleural block using $10 \mathrm{ml}$ of $0.5 \%(\mathrm{~m} / \mathrm{v})$ Bupivacaine. The patient's pain intensity was evaluated using the Numeric Rating Scale (NRS). Any complications that occurred during anaesthesia or recovery were also recorded.

Results: Only ten $(8.3 \%)$ patients had a NRS pain score above three and none had a pain score above five at any time during the 15 hours of postoperative observation. No patients reported a severe pain at all. All patients were fully mobile and were able to eat and drink within 1 hour after surgery. Postoperative nausea and vomiting (PONV) was recorded in 36 patients (29\%) and was significantly associated with morphine consumption. There were no side effects related to intrapleural block. Stanley et al., in a systematic review, concluded that (after taking into account study limitations), the best method of nonnarcotic pain relief following breast augmentation involves pocket irrigation with bupivacaine and ketorolac. (1) Our data suggest that bilateral intrapleural block provides even better pain relief.

Conclusion: Dravid et al., in a review article published in Anaesthesia in 2007, described unilateral breast surgery as an indication for intrapleural anaesthesia. (2) Our experiences suggest that intrapleural anaesthesia can be safely and effectively used in bilateral breast surgery in ERAS pathway.

Disclosure of Interest: None Declared.

\section{PO066}

\section{DECREASED POSTOPERATIVE OPIOID CONSUMPTION FOLLOWING ENHANCED RECOVERY AFTER GYNECOLOGIC SURGERY}

Katherine E. Cain ${ }^{*}{ }^{1}$, Javier D. Lasala ${ }^{2}$, Alpa M. Nick ${ }^{3}$, Larissa A. Meyer $^{3}$, Maria Iniesta-Donate ${ }^{3}$, Terri W. Earles ${ }^{3}$, Mark Munsell ${ }^{4}$, Pedro T. Ramirez ${ }^{3}$

${ }^{1}$ Pharmacy, ${ }^{2}$ Anesthesiology \& Perioperative Medicine, ${ }^{3}$ Gynecologic Oncology and Reproductive Medicine, ${ }^{4}$ Biostatistics, MD Anderson Cancer Center, Houston, United States

Objectives: To determine differences in morphine equivalents in the postoperative period with an enhanced recovery program (ERP) following gynecologic surgery.

Methods: We examined 48 consecutive cases (47 cases were included) post-implementation of our enhanced recovery program and compared these patients to 27 (26 cases were included) historical controls. Patients receiving scheduled opioids prior to surgery at time of anesthesia preoperative assessment were excluded. Pain management for the historical controls varied but included epidurals $(15 \%)$ or patient controlled analgesia (PCA) pumps $(81 \%)+/-$ local injection of liposomal bupivacaine $(15 \%)$. Preemptive analgesia and multimodal pain management strategies were utilized with the primary goal of reducing perioperative opioid administration in our ERP. Preoperatively patients were administered pregabalin, celecoxib, tramadol and acetaminophen. Postoperatively patients were administered scheduled pregabalin, ibuprofen and acetaminophen. Breakthrough pain was treated with oral oxycodone or intravenous hydromorphone. Morphine equivalents were calculated for the first three postoperative days (POD). Wilcoxon rank sum and Fisher exact tests were used for comparison.

Results: The median length of hospital stay post-implementation of our ERP was 3 days. All pre-ERP patients required opioid breakthrough pain medication compared to $81 \%$ in ERP $(p=0.02)$. In total morphine equivalents were reduced by $81 \%$ with implementation of an enhanced recovery program (median $156.5 \mathrm{mg}$ [range 7.5$2534.6 \mathrm{mg}]$ pre-ERP vs. $30 \mathrm{mg}$ [0-230 mg] post-ERP, $\mathrm{p}<0.001)$. When evaluating daily differences (TABLE 1), the greatest reduction in morphine equivalents was noted on POD0 (85\% reduction: preERP $51.7 \mathrm{mg}$ [range $7.5-637.5 \mathrm{mg}$ ] vs. $7.5 \mathrm{mg}$ [range $0-72.5 \mathrm{mg}$ ], $\mathrm{p}<0.001)$.

\begin{tabular}{lccll}
\hline & $\begin{array}{l}\text { Pre-ERP } \\
\text { (Median } \\
\text { [range] })\end{array}$ & $\begin{array}{l}\text { Post-ERP } \\
\text { (median } \\
\text { [range] })\end{array}$ & $\begin{array}{l}\% \\
\text { reduction }\end{array}$ & p-value \\
\hline POD 0 & $51.7[7.5-637.5]$ & $7.5[0-72.5]$ & $85 \%$ & $<0.001$ \\
POD 1 & $57[0-843]$ & $12.5[0-100]$ & $78 \%$ & $<0.001$ \\
POD 2 & $37.85[0-752.6]$ & $7.5[0-72.5]$ & $80 \%$ & $<0.001$ \\
POD 3 & $37.85[0-753]$ & $7.5[0-80]$ & $80 \%$ & $<0.01$ \\
\hline
\end{tabular}

Conclusion: Enhanced recovery effectively reduces postoperative opioid administration in gynecologic surgery patients undergoing exploratory laparotomy. Reductions in opioid related adverse events would be expected to improve perioperative outcomes.

Disclosure of Interest: None Declared. 


\section{PO067}

\section{STRATEGIES TO REDUCE FASTING TIMES IN ENHANCED RECOVERY SURGERY}

Kirstie McPherson ${ }^{*}{ }^{1}$, Sanjiv Patel ${ }^{2}$, Matthew Higham ${ }^{2}$, Monty Mythen ${ }^{2,3}$

${ }^{1}$ Anaesthetics, University College London, ${ }^{2}$ Anaesthetics, University College Hospital, London, ${ }^{3}$ Centre for Anaesthesia, University

College London, London, United Kingdom

Objectives: Enhanced Recovery After Surgery (ERAS) programmes focus on expediting good quality recovery. Preoperatively, avoiding excessive fasting, and carbohydrate loading has been shown to maintain nitrogen balance, reduce postoperative insulin resistance, improve patient wellbeing, immunity and nutrition following surgery $[1,2]$. We were keen to explore patients interpretation of ER fasting recommendations in our own institution, and the impact of local teaching and a didactic perioperative fluid algorithm to support health care workers. We hypothesized that in spite of recommendations, patients still fast excessively.

Methods: Perioperative fluid and hydration status data was collected for all adult patients undergoing general anaesthesia for enhanced recovery surgery over a three day period. This data collection was repeated after a fortnight, following the institution of a local perioperative fluid management strategy and algorithm. Data collection included preoperative fasting times, ingestion of carbohydrate drinks and sips of up to $30 \mathrm{ml}$ of water up to an hour before surgery. Excel was used for analysis of data.

Results:

\begin{tabular}{|c|c|c|}
\hline & Pre algorithm & Post algorithm \\
\hline Data sets $(\mathrm{n})(\%)$ & $56 / 59(95 \%)$ & $47 / 55(85.5 \%)$ \\
\hline $\begin{array}{l}\text { Mean fasting time } \\
\text { (food) (hours) }\end{array}$ & 13.6 & 11.0 \\
\hline $\begin{array}{l}\text { Mean fasting time } \\
\text { (clear fluid) (hours) }\end{array}$ & 6.5 & 4.4 \\
\hline $\begin{array}{l}\text { Carbohydrate drink consumed } \\
\quad \text { (number) }(\%)\end{array}$ & $4 / 56(7.1 \%)$ & $3 / 47(6.4 \%)$ \\
\hline $\begin{array}{l}30 \mathrm{ml} \text { aliquot of } \\
\text { water pre-operatively } \\
\text { (number) }(\%)\end{array}$ & $21 / 56(37.5 \%)$ & $33 / 47(70.2 \%)$ \\
\hline $\begin{array}{l}\text { Drinking in recovery } \\
\text { (number) }(\%)\end{array}$ & $49 / 56(87.5 \%)$ & $39 / 47(83.0 \%)$ \\
\hline PONV (number) $(\%)$ & $3 / 56(5.4 \%)$ & $4 / 47(8.5 \%)$ \\
\hline
\end{tabular}

Conclusion: Fasting times are excessive in spite of ER programmes. Reasons are multifactorial and may be related to anxiety and misinterpretation and communication of information. A more didactic approach may support clinicians and empower patients to actively engage with preoperative fasting guidelines.

Disclosure of Interest: None Declared.

\section{PO068}

\section{THE LONG ROAD TO RECOVERY BUT HOW FAR? ONE CENTRE'S EXPERIENCE OF EARLY MOBILISATION IN ELECTIVE COLORECTAL PATIENTS}

Baseem Choudhry ${ }^{*}{ }^{1}$, Kelvin Wang ${ }^{1}$, Damian Bragg ${ }^{1}$, Charles-Maxwell Armstrong ${ }^{1}$ on behalf of Nottingham University Hospitals Trust

${ }^{1}$ Colorectal Department, Queens Medical Centre, Nottingham, Nottingham, United Kingdom

Objectives: Enhanced recovery after surgery (ERAS) protocol is generally hailed as a progressive policy to expedite recovery. Although early mobilisation is recommended, there is no evidence to support an optimal mobilisation distance or duration to aid recovery. The aim of this study was to assess if there is a distance patients should aim to achieve and whether that impacts on hospital length of stay (LOS).

Methods: We conducted a retrospective observational study on patients who were admitted for an elective colorectal procedure in a large teaching hospital. All patients had undergone preoperative education as per local ERAS policy. A schematic diagram of the ward was created, and was publicised on the ward for the physiotherapists to use and document the distance patients mobilised in the medical notes. This data was then correlated with LOS and complications, and analysed using Student's t-test.

Results: We observed 50 patients who had undergone elective colorectal surgery. Surgical patients who were ambulant on day 1 had a shorter LOS and fewer complications. Furthermore, patients mobilising greater than 50 metres in the first 3 days were discharged sooner and also had fewer complications.

Conclusion: This study confirms that early mobilisation is associated with enhanced recovery. Patients who mobilised $>50$ metres by day 3 were more likely to be discharged sooner and have fewer complications. However, this study was an observational cohort study and as such cause and effect cannot be established. Furthermore, we did not confound for patients' pre-operative exercise tolerance and further work is required to see if this is a patient factor or a resources/staffing issue.

Disclosure of Interest: None Declared. 


\section{PO069}

\section{A QUALITATIVE STUDY EXPLORING EXPERIENCES OF PERIOPERATIVE NUTRITION IN PEOPLE UNDERGOING COLORECTAL SURGERY WITHIN AN ENHANCED RECOVERY AFTER SURGERY PROGRAMME}

Vaneesha Short ${ }^{*}{ }^{1}$, Eileen Sutton ${ }^{1}$, Andy Ness ${ }^{1}$, Steven Thomas ${ }^{2}$, Charlotte Atkinson

${ }^{1}$ NIHR BRU in Nutrition, Diet and Lifestyle, University of Bristol, ${ }^{2}$ Head \& Neck Surgery, University Hospitals Bristol NHS Trust, Bristol, United Kingdom

Objectives: Nutrition is a key component of Enhanced Recovery After Surgery (ERAS) programmes. Studies have previously indicated that nutrition is an important area of ERAS requiring improvement according to patients. This project aimed to explore experiences of perioperative nutrition $(\mathrm{PN})$ in people undergoing colorectal surgery, to identify potential barriers and facilitators to the uptake of PN practices within an ERAS programme.

Methods: Sixteen individuals scheduled for elective colorectal resection participated in a semistructured interview between postoperative day three and hospital discharge. The topic guide was piloted in four participants and developed iteratively throughout the study. Topics included preoperative counselling, carbohydrate loading, fasting, and postoperative nutrition. Demographic and medical data were collected to contextualise experiences. Data were analysed using an inductive thematic approach, and the constant comparison technique was employed during coding. Four transcripts were double coded to ensure coding scheme validity, and a reflexive approach was used throughout the research process by means of a research diary to acknowledge the potential involvement of the researcher, setting and participant characteristics on the data.

Results: Four key themes were observed: preparation, autonomy, relationship with food and the hospital environment. From these themes, barriers to PN practices were identified including lack of information provision, negative experiences of nutritional drinks, coping with changes in perceptions of food due to chemotherapy, operative consequences and stoma placement, and aspects of the hospital structure. Facilitators included the ability of patients to be actively involved in their recovery, staff support, and accessibility and choice of food.

Conclusion: Perceived barriers and facilitators to the uptake of PN practices within an ERAS programme were identified. Changes in practice that target these could improve patients' experiences of nutrition and ensure that the nutrition components of ERAS programmes are implemented.

Disclosure of Interest: None Declared.

\section{PO070}

COLLABORATIVE NON-OPIOID ANESTHETIC PROTOCOL FOR LIMB SALVAGE SURGERY IN COMPLEX MEDICAL PATIENTS - A CASE SERIES OF THREE PATIENTS

Joseph Myers $^{*}{ }^{1}$, Caitlin S. Garwood ${ }^{2}$, Jennifer D. Pukish ${ }^{1}$, John S. Steinberg ${ }^{2}$, Karen K. Evans ${ }^{2}$, Christopher E. Attinger ${ }^{2}$

${ }^{1}$ Anesthesiology, ${ }^{2}$ Plastic Surgery, MedStar Georgetown University Hospital, Washington DC, United States

Objectives: Addressing the current wide variability of anesthetic care, we implemented the use of a protocol which provides general anesthesia with rapid emergence and effective postoperative analgesia for patients undergoing lower limb surgery. Often exhibiting morbid obesity, a requirement for supplemental oxygen, or lengthy procedures, limb salvage patients provide challenging anesthetic management.

Methods: Members of the Departments of Anesthesiology and Plastic Surgery collaborated on a propofol/sevoflurane general anesthetic protocol which required a checklist-driven discussion to formulate a multimodal non-opioid analgesic plan. To be administered before emergence from anesthesia, ketorolac, acetaminophen, and peripheral nerve blocks were considered. These blocks were performed by the surgical team using bupivacaine $0.5 \%$ or bupivacaine liposomal injectable suspension (Exparel) with direct visualization of the nerves in the surgical field. We present three patients who underwent a lower extremity limb salvage procedure (i.e. above knee amputation, calcanectomy, and free flap) using our anesthetic protocol.

Results: After agreement of the surgical and anesthesia teams, each patient received ketorolac $30 \mathrm{mg}$ and acetaminophen $1000 \mathrm{mg}$ IV. Two received peripheral nerve blocks. No opioids were required intraoperatively and all three patients arrived in the PACU with initial numeric pain scores of zero. Opioid requirements during their PACU stay ranged from fentanyl $100 \mathrm{mcg}$ to oxycodone $1 \mathrm{mg}$. One patient received no opioids throughout their perioperative course. No complications were noted. Conclusion: We believe that our study suggests a role for an opioid-free general anesthetic protocol to enhance recovery and improve comfort and safety. Utilizing a collaborative checklistdriven strategy for postoperative analgesia, consistency and efficacy are improved. Particularly useful for patients with multiple comorbidities, a team discussion of bleeding concerns, liver or kidney disease, and the feasibility or risk of a peripheral nerve block are invaluable. Simple to reproduce, our non-opioid anesthetic protocol can be taught to those with limited experience but is effective for the most challenging patients.

\section{PO071}

\section{ERAS PERIOPERATIVE CARE PROTOCOL IN ESOPHAGECTOMY}

Marianna Raevskaya* ${ }^{1}$, Vadim Sizov ${ }^{2}$, Vladimir Kazennov ${ }^{1}$, Sergey Ilyin $^{2}$, Dmirtry Ruchkin ${ }^{3}$ on behalf of Prof. Dr Ruchkin Dmirtry, Sergey Kamnev ${ }^{2}$ on behalf of ERAS A.V. Vishnevsky Institute of Surgery

${ }^{1} \mathrm{ICU},{ }^{2}$ Anesthesiology Department, ${ }^{3}$ Thoracic Depart, A.V. Vishnevsky Institute of Surgery, Moscow, Russian Federation

Objectives: There is evidence of improvement of outcomes after the implementation of Enhance Recovery After Surgery (ERAS) protocols in the gastrointestinal surgery. In our opinion enhancement of outcomes of patients undergoing esophagectomy requires the implementation of such protocol. Although there are no ERAS society recommendations for esophagectomy. Methods: A total of 54 patients (37 males and 17 females) undergoing esophagectomy (McKeown 23; Transhiatal 31) from December 2012 to December 2014. The median age was 56 [48; 65, interquartile range, IQR] years. Surgeries were performed for esophageal cancer, benign stenosis and neuromuscular disease in 31,13 and 7 cases respectively. Management was according to our enhance recovery protocol based on ERAS society principles. The protocol includes preoperative, intraoperative and postoperative approaches. Preoperative: a) routinely counseling by ERAS team b) no prolonged fasting; c) nutrition; d) incentive spirometry education of patients; f) fluid and carbohydrates loading. Intraoperative: a) avoidance of salt and water overload; b) judicious use of vasopressors; c) protective lung ventilation; d) high-thoracic epidural anesthesia/analgesia; d) maintenance of normothermia. Postopearative: a) immediate or early extubation; b) avoidance of salt and water overload; c) daily weight measurement; d) multimodal analgesia; d) early mobilization; e) stimulation of gut motility; f) audit of compliance and outcomes.

Results: The median of ICU stay, postoperative hospital length of stay were $0,8[0,7 ; 1,2]$ and 9 days $[7,5 ; 10], 24$ patients were extubated immediately in the operation room with 2 cases of reintubation. The median of ICU ventilation time was $165 \min [120 ; 325]$. There was one lethal case from embolic stroke. A total 30-day mortality was $1,85 \%$, morbidity $46,3 \%$ There were 13 cases of atelectasis, 6 cases of pleural effusion that required drainage, 3 cases of $\mathrm{PaO}_{2} / \mathrm{FiO}_{2}<300$ $\mathrm{mmHg}$ on the first postoperative day and 5 cases of pneumonia (different cases).

Conclusion: Presented enhance recovery protocol for esophagectomy promotes good outcomes and low mortality rate compared to other centers.

Disclosure of Interest: None Declared.

\section{PO072}

\section{THE EFFECT OF PREOPERATIVE CARBOHYDRATE LOADING ON POSTOPERATIVE RECOVERY FOLLOWING TOTAL KNEE AND HIP ARTHROPLASTY}

\author{
Katie Lyon ${ }^{*}{ }^{1}$, Alice Young ${ }^{2}$, David McDonald ${ }^{1}$, Mike Gill ${ }^{3}$
}

${ }^{1}$ Rehabilitation, ${ }^{2}$ Clinical Nutrition \& Dietetics, ${ }^{3}$ Department of Anaesthetics, Golden Jubilee National Hospital, Glasgow, United Kingdom

Objectives: Enhanced Recovery Programmes (ERP) are established evidence based pathways fo improving clinical outcomes after joint replacements. Pain, dizziness and general weakness remain an issue post-operatively (1). Orthostatic intolerance (2) or vasovagal syncope is another issue which delays early mobilisation. Carbohydrate $(\mathrm{CHO})$ preloading is suggested to improve insulin resistance associated with protein breakdown and muscle loss (3). Hence by providing a $\mathrm{CHO}$ preload, these issues may be addressed. However there is little evidence to support $\mathrm{CHO}$ preloading in elective orthopaedics (4). The objective was to determine the effect of preoperative CHO loading on postoperative recovery in primary total knee or hip replacement.

Methods: A retrospective audit was carried out between July-September 2012. A consecutive series $(n=134)$ undergoing primary THR or TKR were reviewed. 58 patients under two surgeons received three $12.5 \%$ carbohydrate Preload (Vitaflow ${ }^{\mathrm{TM}}$ ) mixed with $400 \mathrm{ml}$ of water the nigh before and morning of surgery (CHO Group). 76 patients under two other surgeons were in the standard care group.

Results: 3 patients in the $\mathrm{CHO}$ group experienced a vasovagal syncope on first mobilisation compared to nil in the standard care group $(\mathrm{p}<0.05)$. Postoperative catheterisation rates were higher in the $\mathrm{CHO}$ group $(10.3 \%$ vs. $5.3 \%)$. Fewer $\mathrm{CHO}$ group patients experienced nausea and vomiting on theatre night and following day. No difference was noted in analgesic requirements, VRS scores and length of stay were similar for both groups (4.4 vs. 4.0 days).

Conclusion: Preoperative CHO loading has no effect on incidence of vasovagal, postoperative analgesia or length of stay yet lower nausea and vomiting scores. Preoperative CHO intake resulted in higher rates of urinary catheterisation - this may be linked with the $1200 \mathrm{mls}$ fluid intake. Preoperative $\mathrm{CHO}$ loading for joint arthroplasty does not improve postoperative recovery or reduce incidence of vasovagal syncope as part of an enhanced recovery programme.

Disclosure of Interest: None Declared

Disclosure of Interest: None Declared. 


\section{PO073}

\section{RISK OF ANASTOMOTIC LEAKAGE WITH NON- STEROIDAL ANTI-INFLAMMATORY DRUGS WITHIN AN ENHANCED RECOVERY PROGRAM}

Nathalie Bakker ${ }^{*}{ }^{1}$, Jort D. Deelder ${ }^{1}$, Milan C. Richir ${ }^{1}$, Hamit Cakir ${ }^{1}$, Hiëronymus J. Doodeman ${ }^{1}$, Wilhelmina H. Schreurs ${ }^{1}$, Alexander P. Houdijk ${ }^{1}$

${ }^{1}$ Surgery, Medical Center Alkmaar, Alkmaar, Netherlands

Objectives: Anastomotic leakage is a serious complication after colorectal resection. Recent studies suggest that non-steroidal antiinflammatory drugs may increase the risk of anastomotic leakage. ${ }^{1}$ The aim of the present study was to investigate the relation between postoperative use of NSAIDs and the occurrence of anastomotic leakage (AL) in an enhanced recovery population.

Methods: Patients undergoing an elective colon or rectal resection with primary anastomosis because of malignancy and treated within our enhanced recovery program were included. Uni- and multivariable logistic regression analysis was used to determine the association between NSAIDs and other risk factors on the occurrence of AL.

Results: Between 2006 and 2013, 856 colorectal resections met the inclusion criteria. Overall the AL rate was significantly higher in the group that received NSAIDs compared to patients who did not receive NSAIDs: $9.0 \%$ vs. $5.3 \%, \mathrm{p}=0.047, \mathrm{OR}=1.77$. This higher rate was only seen in patients that recieved diclofenac: $\mathrm{AL}$ in colonic resections: $11.8 \%$ vs. $6.0 \%, \mathrm{OR}=2.09, \mathrm{p}=0.016$, $\mathrm{AL}$ in rectal resections: $11.9 \%$ vs. $0 \%, p=0.030$. Only male sex (OR 2.33, $\mathrm{p}=0.003$ ) was also independently associated with AL.

Conclusion: The results of this study are in line with other comparable studies in the literature, showing an increased risk for AL with COX-2 selective NSAIDs and NSAIDs with a high COX-2 selectivity like diclofenac. The use of diclofenac in colorectal surgery can no longer be recommended. Alternatives for postoperative analgesia need to be explored within an enhanced recovery program.

Disclosure of Interest: None Declared.

\section{PO074}

\section{A SUCCESSFUL IMPLEMENTATION OF ERAS PROGRAM}

Astrid Langenes ${ }^{1}$, Helena $\AA^{\prime}$ lev $^{*}{ }^{1}$ on behalf of ERAS Group of Danderyds Hospital

${ }^{1}$ Surgery, Danderyds sjukhus, Stockholm, Sweden

Objectives: Fast-track surgery, (Kehlet) was implemented at Danderyd's Hospital in the early twentieth century but the guidelines were not performed fully. In 2003, the ERAS care program was put into practice, but the compliance was not optimal. In autumn 2009 a quality improvement project was initiated in order to increase compliance to the ERAS program. A multidisciplinary group from different departments at the hospital was set up. The goal of the project was to systematically reintroduce ERAS in order to establish best treatment and gain control of the improvement and outcome. In connection with the formation of the working group, we learned about the ERAS implementation program. This group was initiated and led by a professor at the Department of Surgery at the University Hospital in Örebro Olle Ljungkvist. The program included assistance to identify the parts of the ERAS program, which was not functioning optimally and the possibility of registering patients in the ERAS Interactive Audit System. The proportion of laparoscopic surgeries increased gradually during this period, which led to a new pain management strategy. New guidelines and checklists were developed and introduced to the rest of the staff. The group hired a nurse to follow up the program.

Methods: A prospective registration of perioperative data of all patients passing the program between 2010 and 2013.

Results: Follow-up and evaluation was retrieved from ERAS interactive audit system; Analysis \& Reports. It showed earlier postoperative mobilization of the patients. Compliance to ERAS parameters increased from $64 \%$ to $72 \%$ and the median length of stay (LOS) decreased with one day after rectum and colon surgery. Postoperative complications: wound infections, pain, nausea and vomiting were reduced with $30-40 \%$.

Conclusion: The increased adherence to the treatment program is believed to be due to a number of success factors such as; ERAS nurse, regular multidisciplinary meetings with focus on improvement. Increasing proportion of TAP blocades to the laparoscopic colorectal resections led to reduced pain and increased ability to mobilize and reduced LOS.

Disclosure of Interest: None Declared. 


\section{PO075}

\section{BUNDLED PAYMENT AND ENHANCED RECOVERY AFTER SURGERY (ERAS)}

Jeffrey Huang* 1

${ }^{1}$ Winnie Palmer Hospital, Orlando, United States

Objectives: This review is to prove that ERAS is the essential practice model for bundled payment for surgical procedures.

Methods: Literature reviews.

Results: ERAS and Bundled payment system share many common grounds.

\begin{tabular}{|c|c|c|}
\hline & Bundled payment & ERAS \\
\hline goal & $\begin{array}{l}\text { higher quality, more } \\
\text { coordinated care at a } \\
\text { lower cost }\end{array}$ & $\begin{array}{l}\text { Improve surgical quality } \\
\text { with lower cost }\end{array}$ \\
\hline Care Team & $\begin{array}{l}\text { coordinated, } \\
\text { interdisciplinary }\end{array}$ & $\begin{array}{l}\text { coordinated, } \\
\text { interdisciplinary }\end{array}$ \\
\hline Protocols & Standardized & standardized \\
\hline $\begin{array}{l}\text { Monitoring } \\
\text { Metris }\end{array}$ & $\begin{array}{l}\text { standard adverse event } \\
\text { reporting measures, } \\
\text { reoperation, and } \\
\text { readmission rates, } \\
\text { HCAPS scores }\end{array}$ & $\begin{array}{l}\text { Length of stay, } \\
\text { complications, } \\
\text { readmission rates, } \\
\text { patient satisfaction }\end{array}$ \\
\hline Practice & Evidence based & Evidence based \\
\hline Audit system & Required & required \\
\hline $\begin{array}{l}\text { Continuous } \\
\text { improvement }\end{array}$ & Required & required \\
\hline Procedures & Listed procedures & $\begin{array}{l}\text { ERAS protocols for major } \\
\text { procedures included all } \\
\text { listed procedures in } \\
\text { bundled payment }\end{array}$ \\
\hline $\begin{array}{l}\text { Outcomes } \\
\text { (clinical and } \\
\text { economic) }\end{array}$ & Unknown & Proven by many RCTs \\
\hline Model & Payment & Practice \\
\hline
\end{tabular}

Conclusion: ERAS has most elements of requirements for successful implementation of bundled payment for surgical procedures. Evidence from randomized controlled trials (RCTs), systematic reviews, and meta analyses has demonstrated that ERAS can improve healthcare quality with lower cost. ERAS society has published many clear guidelines for all members of the clinical team (anesthetists, surgeons, physiotherapists, dietitians, and nursing staff). Successful implementation of ERAS pathways have been reported following a brief preparatory period, emphasizing the need for staff and patient education $(1,2)$. Therefore ERAS is the essential practice model for bundled payment for surgical procedures.

Disclosure of Interest: None Declared.
PO076

\section{DEVELOPMENT OF A COLORECTAL BUNDLE TO PREVENT SURGICAL SITE INFECTIONS: CONSENSUS BUILDING AND IMPLEMENTATION STRATEGIES FOR A SUCCESSFUL TEAM APPROACH}

Stephen Brandstetter ${ }^{*}{ }^{1}$, Mark Horattas ${ }^{1}$

${ }^{1}$ General Surgery, Akron General Medical Center, Akron, United States

Objectives: Bundles have been created at numerous institutions to standardize certain aspects of perioperative care in surgical practice. Institutional Surgical Site Infection (SSI) rates at our institution were unacceptably high. A multi-departmental team created a "colorectal bundle" implemented in the fourth quarter of 2014. We encountered several unanticipated difficulties in both creation and implementation of a Preventative Colorectal SSI Bundle at our institution.

Methods: From October 1 to December 1, 2014, members from multiple departments created a bundle care system pertaining to minimize risks of infection including aspect of preoperative care, anesthesia, operating room, post-anesthesia and post-operative care for colorectal procedures. We also surveyed caregivers at all levels of care to assess other issues found during this process.

Results: The feedback of care-givers has given our group insight into more efficient implementation strategies. We have developed a very successful program with widespread support, and have found ways to improve the timing and the perception of a bundled care rollout.

Conclusion: The management of preventable SSI requires the effort and cooperation of all hospital staff involved in preoperative, intraoperative, and post-operative care of patients. The hurdles and obstacles encountered and resolved at our institution offer many lessons to others developing bundles to decrease their rate of SSI after colorectal surgery with a comprehensive team approach.

Disclosure of Interest: None Declared. 


\section{PO077}

\section{A FOUR-YEAR EXPERIENCE WITH THE APPLICATION OF AN ERAS PROGRAM IN PATIENTS UNDERGOING MAJOR INTRA-ABDOMINAL SURGICAL PROCEDURES}

Vasileios Alivizatos ${ }^{*}$, Pavlos Athanasopoulos ${ }^{2}$, Fotis Kadjianis ${ }^{2}$, Nikolaos Apostolopoulos ${ }^{2}$

${ }^{1}$ Surgery, Artificial Nutrition and Intestinal Failure Unit, ${ }^{2}$ Surgery, "St. Andreas" General Hospital, Patra, Greece

Objectives: Major abdominal operations lead to suppression of the immune functions and increased susceptibility to postoperative complications and longer length of hospital stay. The aim of this study was to present our 4-year experience with the application of an ERAS program and to evaluate whether it is associated, when compared to traditional care, with decreased morbidity and improved outcome in patients undergoing major intra-abdominal surgery.

Methods: All consecutive patients with an ASA grade $\leq 3$ undergoing elective major intraabdominal surgical procedures during a time period of 4 years (Sept. 2010-Aug. 2014) and treated according to an ERAS regime were prospectively studied and compared with patients treated with the conventional perioperative care during a 6-month period (Sept. 2010-Febr. 2011). The outcome measures were incidence of postoperative complications, length of postoperative hospital stay, number of relaparotomies, number of readmissions and mortality within 30 days. The protocol of the study has been approved by the Scientific and Ethical Committee of our Hospital and it has been registered through the NIH trials registration system (ClinicalTrials.gov NCT01400711). Data analysis was done by the use of Fisher's exact test and Student's t-test. Results: Sixty one patients treated according to the ERAS program were compared with 38 controls who had conventional perioperative care. The two groups were similar with respect to age, gender, ASA grade, type of disease and type of surgery. There was a significantly lower incidence of postoperative complications in the ERAS group compared to the control group $(13.1 \%$ vs $42.1 \%$ respectively, $p=0.001)$. Length of stay was also significantly shorter in the ERAS group compared to the control group $(6.09 \pm 3.9$ days vs $12.2 \pm 5.9$ days respectively, $\mathrm{p}<0.0001$ ). There was no difference between the ERAS and the control group for readmission rate, relaparotomies and mortality.

Conclusion: The results of this study suggest that, in selected patients undergoing major intraabdominal surgery, the application of an ERAS program is associated with significantly lower incidence of postoperative complications and with significantly shorter length of hospital stay.

Disclosure of Interest: None Declared.

\section{PO078}

\section{ERAS OPTIMIZES OUTCOMES OF OPEN RADICAL CYSTECTOMY FOR BLADDER CANCER - EFFECTIVENESS OF A PILOT STUDY}

\author{
Yongwei Lim ${ }^{*}{ }^{1}$, Christopher W. S. Cheng ${ }^{1}$, Lui Shiong Lee ${ }^{1}$ \\ ${ }^{1}$ Urology, Singapore General Hospital, Singapore, Singapore
}

Objectives: Radical cystectomy may be associated with morbidity, which may be attenuated with enhanced recovery after surgery (ERAS) protocols. This study looks at the effectiveness of implementing ERAS following open radical cystectomy, and the perioperative outcomes. Methods: ERAS protocol were implemented from $1^{\text {st }}$ January 2013 to $31^{\text {st }}$ December 2014. Suitable patients on conventional surgery protocol (CS) were identified between $1^{\text {st }}$ January 2011 to 31 st December 2012 as historical controls. The outcomes compared between the CS and ERAS cohort include operative complications, pathological outcomes, estimated blood loss (EBL), time of ileus (tolerance of solid food), and length of stay (LOS). The elements of ERAS include permitting clear feeds up to two hours before surgery, no mechanical bowel preparation, early removal of nasogastric tube, prevention of post-operative nausea and vomiting, early feeding, deep vein thrombosis prophylaxis and early ambulation.

Results: There were $\mathrm{n}=20(\mathrm{CS})$ and $\mathrm{n}=8$ (ERAS) patients respectively. The compliance to ERAS was $100 \%$. The pathological stage of CS patients was pTcis $10 \%(\mathrm{n}=2 / 20)$, pTa $5 \%$ $(\mathrm{n}=1 / 20)$, pT1 10\% $(\mathrm{n}=2 / 20)$, pT2 35\% $(\mathrm{n}=7 / 20)$, pT3 25\% $(\mathrm{n}=5 / 20)$ and $\mathrm{pT} 415 \%$ $(\mathrm{n}=3 / 20)$. The ERAS patients were pT1 25\% $(\mathrm{n}=2 / 8)$, pT2 25\% $(\mathrm{n}=2 / 8)$, pT3 38\% $(\mathrm{n}=3 / 8)$ and pT4 $12 \%(\mathrm{n}=1 / 8)$. The median EBL was $600 \mathrm{ml}(\mathrm{CS})$ and $800 \mathrm{ml}$ (ERAS) (mean 726 (CS), 1112 (ERAS) and range 200-2000 (CS), 400-3000 (ERAS)). The complication rate (Clavien-Dindo III) was $\mathrm{n}=2 / 20(10 \%)(\mathrm{CS})$ and $\mathrm{n}=1 / 8(12.5 \%)$ (ERAS). The median time of ileus was 6 days (CS) and 4 days (ERAS) (mean 5.8 (CS), 4.3 (ERAS) and range 3-9 (CS), 3-6 (ERAS) $(\mathrm{p}=0.64))$. The median length LOS was $10(\mathrm{CS})$ and 7.5 (ERAS) days (mean 13 (CS) and 8.6 (ERAS), range 6-39 (CS) and 6-16 (ERAS ( $\mathrm{p}=0.08$ ). Readmission rate within 30 days was $30 \%(n=6 / 20(C S)$ and $12.5 \%(n=1 / 8$ (ERAS). Readmission after CS were for poor intake $(n=3 / 20)$, ileus $(n=1 / 20)$, pyelonephritis $(n=1 / 20)$ and pneumonia $(n=1 / 20)$. Readmission after ERAS was for poor intake $(n=1 / 8)$.

Conclusion: Although it is a small study with a non-randomised comparison, ERAS is feasible and possibly associated with shorter ileus time and LOS
PO079

\section{REGIONAL ANAESTHESIA WITHIN AN ENHANCED RECOVERY PROTOCOL FOR GYNAECOLOGICAL SURGERY IS OPIOID-SPARING AND PROVIDES HIGH LEVELS OF PATIENT SATISFACTION \& COMFORT}

Arun Nair ${ }^{*}{ }^{1}$, Joanne Lee ${ }^{2}$, Ian Lyons ${ }^{1}$, Victoria White ${ }^{2}$, Catherine Brocklehurst $^{2}$, Sian Davies ${ }^{1}$, Anish Bali ${ }^{2}$, Martyn Traves ${ }^{1}$

${ }^{1}$ Anaesthesia, ${ }^{2}$ Gynaecology, Royal Derby Hospital, Derby, United Kingdom

Objectives: Opioid analgesics have many side-effects which may reduce patient satisfaction and motivation during recovery. Regional anaesthesia (spinal/epidural) forms an integral part of our enhanced recovery (ERAS) protocol for gynaecological surgery and we assessed if it reduced opioid requirement and its effect on patient experience.

Methods: Consecutive cases undergoing gynaecological procedures requiring general anaesthesia, excluding wide local excision or pelvic floor surgery were analysed from patient records. Our preliminary study included 50 cases and the subsequent follow-up audit included 263 . Patient satisfaction questionnaires were completed prior to discharge and patients were followed up by telephone. $144(55 \%)$ questionnaires were returned. Data were analysed using Microsoft Excel. Results: Following a preliminary study, promotion of the ERAS protocol increased use of regional anaesthesia from $55 \%$ to $88 \%$. Prescription of simple analgesia had not significantly changed. Regional techniques reduced IV opioid administration under general anaesthesia from $98 \%$ to $50 \%$ (Fisher's exact test, $\mathrm{p}<0.001 ; \mathrm{n}=40$ and 223 , respectively). Perioperative provision of simple analgesia had not significantly changed. Opioid use in the post-anaesthesia care unit (PACU) was reduced; $65 \%$ (26/40) patients not receiving a regional technique required opioids compared with $20 \%(44 / 223)$ who received a regional technique $(\mathrm{p}<0.001)$. Of 125 patients undergoing laparoscopic hysterectomy, use of regional techniques reduced PACU opioid use from 52\% (13/25) to $7 \%(7 / 100 ; \mathrm{p}<0.0001)$. Regional anaesthesia reduced moderate/severe discomfort from $43 \%$ to $23 \%$ in PACU ( $\mathrm{p}=0.01, \mathrm{n}=28$ and 107 respectively). A non-significant reduction in nausea was also noted. Overall satisfaction was high in PACU $(>85 \%)$ and was maintained on follow-up. Following discharge from PACU, opioid use was not significantly different.

Conclusion: These data highlight the benefits of regional anaesthesia in reducing perioperative opioid use and increasing patient comfort. Regional anaesthesia may facilitate recovery and contribute to high levels of patient satisfaction as part of an ERAS protocol.

Disclosure of Interest: None Declared.

\section{PO080}

\section{ERAS PROTOCOLS IN COLORECTAL SURGERY: IMPLEMENTATION PROBLEMS}

Riza Haldun Gundogdu ${ }^{*}{ }^{1}$, Pamir E. Ersoy ${ }^{1}$, Soner Akbaba ${ }^{1}$, Bahadir O. Bozkirli ${ }^{2}$, Omer Yazicioglu ${ }^{1}$

${ }^{1}$ General Surgery, Ataturk Training and Research Hospital, ${ }^{2}$ General Surgery, Ankara Training and Research Hospital, Ankara, Turkey

Objectives: The ERAS protocols suggest evidence based current variations instead of conventional practice. We have been carrying out the protocols in most of the colorectal surgery cases since 2006 in our clinic. A study was planned to evaluate the results of our ERAS protocols in terms of compliance, item by item.

Methods: We included 160 colorectal operations, which were performed according to ERAS protocols. For all patients, the length of stay, the timing of oral intake, gastrointestinal tolerance, morbidity and, mortality were recorded.

Results: 18 components of ERAS were applied for the patients. Median age was 57.4 (3386). The mean time for length of stay for the uncomplicated patients $(n=129)$ was 7,2 days. The overall compliance of the protocol was $90.6 \%$. The most difficult items to accomplish were epidural anesthesia/analgesia, intraoperative fluid management and abdominal drain policy.

Conclusion: The application of fast track protocols does not add risk to our patients in terms of complications, morbidity and mortality. In some patients it is difficult to reach the full protocol compliance. The rates of compliance are much better than the ones in the literature. In consequence of this study, we concluded that ERAS protocols for elective colorectal surgery is applicable on trust and decided to maintain the procedure. However, we should pay special attention to three of them.

Disclosure of Interest: None Declared. 


\section{PO081}

\section{POSTOPERATIVE FLUID PRESCRIBING PRACTICES;} COMPLIANCE IN ENHANCED RECOVERY

Kirstie Mcpherson ${ }^{*}$, Matthew Higham ${ }^{1}$, Sanjiv Patel ${ }^{1}$, Monty Mythen $^{2}$

${ }^{1}$ Anaesthetics, University College Hospital, London, ${ }^{2}$ Anaesthetics, Centre for Anaesthesia, London, United Kingdom

Objectives: Enhanced recovery (ER) pathways are well established. Optimising fluid therapy is an important element in ER, postoperatively promoting enteral route and removing intravenous fluids (i.v.) promptly [1]. Delayed discharge is associated with deviation from postoperative elements, including continued i.v. fluids after day 1 [2]. We wanted to look at postoperative compliance, including early enteral intake, discontinuation of i.v. fluid, and type of fluid prescribed. We anticipated that introduction of a fluid algorithm and aide-memoire reflecting ER principles would reduce deviation in our institution.

Methods: We collected data for all surgical patients undergoing ER over a 3day period in our institution, and recorded all fluid intake for a minimum of 24 hours postoperatively. Variables included type, volume and duration of postoperative fluids and fulfilment of ER principles to remove i.v. fluids as early as possible, and promote enteral route intake. The same method of data collection was repeated following the institution of local teaching and introduction of a perioperative didactic fluid algorithm. Data was analysed in excel. Results:

\begin{tabular}{|c|c|c|c|}
\hline \multicolumn{2}{|l|}{ Post operative ER element } & \multirow{2}{*}{$\begin{array}{l}\begin{array}{l}\text { Pre-algorithm } \\
(\mathrm{n}=56)\end{array} \\
\mathrm{n}=38(67.9 \%)\end{array}$} & \multirow{2}{*}{$\begin{array}{l}\begin{array}{l}\text { Post-algorithm } \\
(\mathrm{n}=47)\end{array} \\
\mathrm{n}=23(48.9 \%)\end{array}$} \\
\hline Postoperative & None & & \\
\hline Fluid & Hartmann's & $\mathrm{n}=18$ & $\mathrm{n}=15$ \\
\hline \multirow[t]{7}{*}{$\begin{array}{l}\text { Prescribed; n, total volume } \\
\text { (mean; ml/pt) }\end{array}$} & & $\begin{array}{l}22,8001 \\
(1267 \mathrm{ml})\end{array}$ & $\begin{array}{l}14,3001 \\
(953 \mathrm{ml})\end{array}$ \\
\hline & $0.9 \% \mathrm{NaCl}$ & $\mathrm{n}=1$ & $\mathrm{n}=1$ \\
\hline & & $\begin{array}{l}500 \mathrm{ml} \\
(500 \mathrm{ml})\end{array}$ & $\begin{array}{l}1100 \mathrm{ml} \\
(1100 \mathrm{ml})\end{array}$ \\
\hline & Dex:Saline & $\mathrm{n}=0$ & $\mathrm{n}=8$ \\
\hline & & $0 \mathrm{ml}$ & $\begin{array}{l}8000 \mathrm{ml} \\
(1000 \mathrm{ml})\end{array}$ \\
\hline & $5 \%$ & $\mathrm{n}=0$ & $\mathrm{n}=1$ \\
\hline & dextrose & $0 \mathrm{ml}$ & $\begin{array}{l}1000 \mathrm{ml} \\
(1000 \mathrm{ml})\end{array}$ \\
\hline \multicolumn{2}{|c|}{$\begin{array}{l}\text { Average volume post operative i.v. fluid } \\
\text { prescribed (ml/patient) }\end{array}$} & $1294 \mathrm{ml}$ & $1017 \mathrm{ml}$ \\
\hline \multicolumn{2}{|l|}{$\begin{array}{l}\text { Duration postoperative fluid } \\
\text { (mean; hours) }\end{array}$} & 13.4 hours & 8.9 hours \\
\hline \multicolumn{2}{|c|}{$\begin{array}{l}\text { Patient eating and drinking at } 24 \text { hours } \\
\text { postoperatively number }(\%)\end{array}$} & $55(98.2 \%)$ & $46(97.9 \%)$ \\
\hline \multicolumn{2}{|c|}{ PONV at 24 hours number ( $\%)$} & $2(3.6 \%)$ & $0(0 \%)$ \\
\hline
\end{tabular}

Conclusion: Reinforcing the principles of ER with local teaching and simple algorithms can lead to a change in fluid prescribing practices. Mindful behaviours in type, duration and volume of maintenance fluid type prescribed are likely to promote greater compliance, reducing deviation and improving outcomes.

Disclosure of Interest: None Declared. 